\title{
A Practical Approach to Interpretation of CBF Measured by Mean of Xenon-CT in Patients with Traumatic Brain Injury
}

\author{
Arturo Chieregato $^{*},{ }^{1}$, Alessandra Tanfani ${ }^{1}$ and Enrico Fainardi ${ }^{2}$ \\ ${ }^{1}$ U.O. Anestesia e Rianimazione, Rianimazione per la Traumatologia e le Neuroscienze, Dipartimento Emergenza, \\ Ospedale Bufalini, AUSL-Cesena, Cesena, Italy \\ ${ }^{2}$ U.O. Neuroradiologia, Dipartimento di Neuroscienze, Azienda Ospedaliera Universitaria, Arcispedale S. Anna, \\ Ferrara, Italy
}

\begin{abstract}
The measurement of cerebral blood flow (CBF) in traumatic brain injury (TBI) by means of Xenon-CT (XeCT) has been part of the clinical practice of several centres since 1980. Xe-CT consists of a CBF image coupled with CT which offers the possibility to associate morphologic features given by CT with functional information. Today, Xe-CT, in association with Positron Emission Tomography (PET), represents the gold standard for clinical quantitative measurement of CBF. More recently, Xe-CT has improved its potential application in the intensive care unit by means of portable CT scanner. The aim of the present review is to describe the practical experience of a single center with over five hundred XeCT studies obtained in more than two hundred TBI patients, to provide a method to guide the lecture of images at bedside, to present illustrative cases and to review the literature published so far on Xe-CT and TBI. The physiological and clinical variables which may be useful to explain global and regional CBF are detailed. Global CBF and its relationship with ageing, the natural time course in TBI, and the dependency on physiological variables and therapy are discussed. The coupling of global CBF with cerebral metabolic rate of oxygen (CMRO2) and the relationship of arterial venous oxygen content differences (AVDO2) with patient outcome are analyzed. Finally, the current knowledge on regional CBF found in the most important traumatic lesions (posttraumatic cerebral infarction, contusion/laceration, acute subdural hematomas, and intraparenchymal hematoma) is described.
\end{abstract}

Keywords: Arteriovenous difference of oxygen, cerebral blood flow, cerebral metabolic rate of oxygen, ischemia, outcome, traumatic brain injury, Xenon CT.

\section{INTRODUCTION}

The measurement of cerebral blood flow (CBF) in traumatic brain injury (TBI) has always been an objective for clinicians. In fact, physiological data suggest that CBF drop may be associated with ischemia. Furthermore, most of the current therapies act on CBF by manipulating vessel resistance directly (e.g. $\mathrm{PaCO}_{2}$ and indomethacin), indirectly (e.g. mannitol), by means of coupling with metabolic depression (sedative, barbiturate, propofol, hypothermia), or by modifying the cerebral perfusion pressure (CPP), especially in cases with defective autoregulation.

However, technologies measuring CBF were not available for several years for every day clinical application. Xenon computed tomography (Xe-CT) consists of a CBF image coupled with $\mathrm{CT}$ which offers the possibility to associate morphologic features given by CT with functional information. It is a technique that has been available since the early eighties [1,2]. Its application has often been limited by its location outside the intensive care unit (ICU) as well as by the not common immediacy of its measurement in relation to insults. More recently, the potential application of Xe-CT in ICUs has improved due to the introduction of a portable CT scanner [3]. It is now clear, however, that the measurement of CBF is not enough to understand the physiology of TBI

*Address correspondence to this author at the Unità Operativa Anestesia e Rianimazione, Ospedale "M. Bufalini", Viale Ghirotti, 286; 47023 Cesena; Italy; Tel: 39547 352197, 390547 352818/352612; Fax 390547 645008; E-mails: achiere@ausl-cesena.emr.it, achiere@alice.it and predict ischemia, at least when $\mathrm{CBF}$ measurement is performed outside the ICU [4]. Moreover, recent investigations have increasingly emphasized the relevance, after the early phases, of non-ischemic damage in TBI [5]. Once adequately treated in the first 24 hours and managed according to standard advanced NeuroICU care, most TBI patients seem to be affected prominently by a structural and metabolic disorder. In this context, CBF measurement should ideally be coupled with global and regional metabolic measurement. In particular, Xe-CT image should be co-registered with functional magnetic resonance imaging (MRI) and with microdialysis or sensors of perfusion, $\mathrm{Pt}_{\mathrm{i}} \mathrm{O} 2$ [6], thermaldiffusion measurement of regional CBF (TD-rCBF) [7,8] placed in selected brain regions. Xe-CT remains the most consistent alternative to positron emission tomography (PET) to quantitatively measure CBF. In fact, even if PET provides detailed physiological data [9], it is too complex to be widely applied. In spite of its limitations, Xe-CT, today, allows clinicians "to see" and "touch with their hand" what was merely speculatively imagined for a long time: the CBF. Consequently, the aim of this review was to describe a practical approach applied on a daily basis in a NeuroICU by performing Xe-CT measurements in a clinical setting currently consisting of standard monitoring of intracranial pressure (ICP), CPP, and jugular bulb saturation ( $\mathrm{SjO} 2)$.

\section{What is Xenon-CT?}

$\mathrm{Xe}-\mathrm{CT}$ is an imaging technique that quantitatively measures $\mathrm{CBF}$ with a high spatial resolution (approximately 3-4 


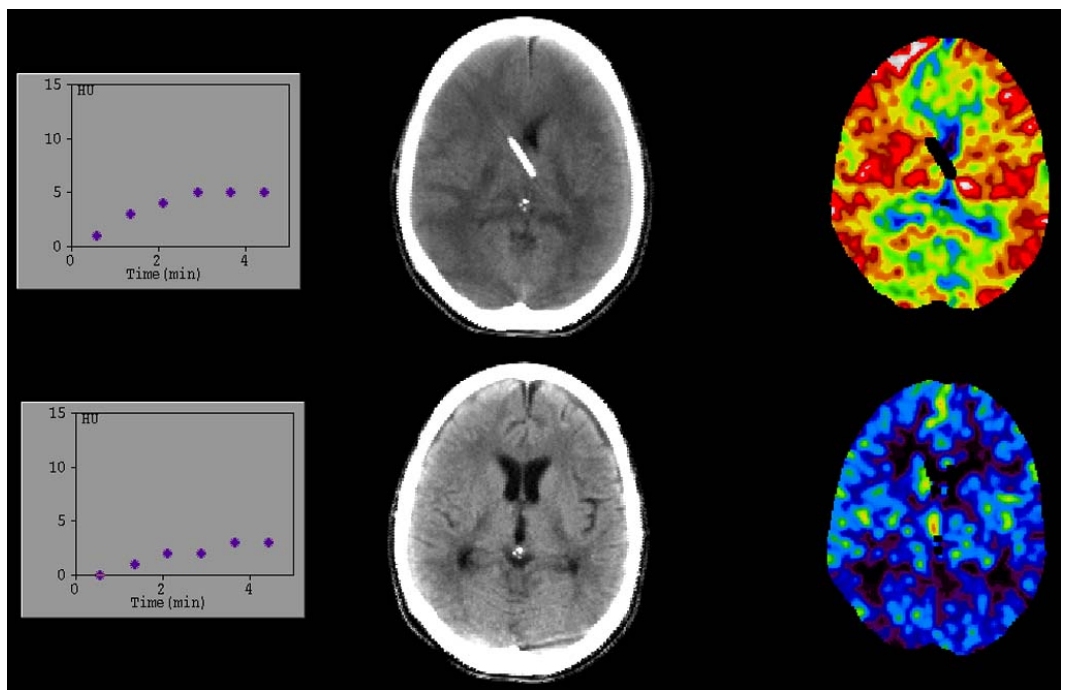

Fig. (1). An example of the basic concept of Xe-CT CBF measurement. In the same patient two different studies obtained on two different days along the acute phase. For each study are reported the CT and the CBF map in association with the time course of Hounsfield Unit (HU) values measured in a pixel located in the same brain region, during the wash-in phase. In the study at the top, a higher CBF is associated with a wider change in $\mathrm{HU}$ in comparison to the changes observed in the study, at the bottom, which are associated with a lower CBF. The extent in the change of $\mathrm{HU}$ also depend on $\mathrm{CBF}$.

$\mathrm{mm})$. In 1945, Kety and Schmidt [10] introduced the nitrous oxide method for the quantitative measurement of CBF in humans. This pioneer technique was based on the Fick principle which states that the amount of a tracer in a tissue region is equal to the amount supplied by arterial blood minus the amount drained by venous blood. The same principle can be applied to stable the Xenon method, since the rate of uptake and clearance of an inert diffusible gas is proportional to blood flow in tissue. The agreement between the Kety Schimdt technique and Xe-CT was fairly good [11]. The reliability of Xe-CT has finally been established by means of comparison with radiolabelled microspheres methods $[12,13]$.

Every CT scanner can be equipped for Xe-CT CBF imaging (Xe/TC system-2 ${ }^{\mathrm{TM}}$, Diversified Diagnostic Products, Inc., Houston, TX). In our Department, CBF studies were conducted from 2000 to 2005 using a Picker 5000 CT scanner (Picker Medical Imaging, Cleveland, $\mathrm{OH}$ ) and thereafter with a Philips Brilliance CT 6 slices Air Scanner (Philips International B.V., Amsterdam, The Netherlands). According to Pindzola and Yonas [14], wash-in protocol is performed using four contiguous axial sections separated by 20 $\mathrm{mm}$ intervals, located on the cerebral hemispheres, with the head aligned along the orbitomeatal plane. Two baseline scans separated by a time interval of 30 seconds are obtained at each of the four chosen levels. After a delay of 33 seconds and during approximately 4.5 minutes, six additional Xenonenhanced scans are obtained at each level while the patient inhaled a mixture of $28 \%$ Xenon, $40 \%$ Oxygen and $32 \%$ room air. A reduction in Xenon concentration to $28 \%$ seemed appropriate to achieve an adequate signal-to-noiseratio for quantitative $\mathrm{CBF}$ calculation and to minimize flow activation [15]. During the two basal scans, the software records the Hounsfield value for each pixel. In the six additional Xenon-enhanced scans, the changes in Hounsfield values during the diffusion of the Xenon in the brain tissue are calculated. The difference between the baseline and the inhalation values (Fig. 1) is a function of the CBF values according to the Fick principle. The six enhanced scans are useful while waiting for the inhaled Xenon to reach an equilibrium with pulmonary arterial capillaries and to improve the accuracy of the final data through multiple measurements. The comparability of the values obtained by Xe-CT apparatus (from site to site and from scanner manufacturer to manufacturer) is possible by testing with phantom values [16].

The availability of an estimated value of arterial Xenon (Fig. 2) allows the quantitative calculation of CBF values. Several calculations are involved in the resolution of the CBF equation, derived from the Fick's calculation. Among the variables affecting final $\mathrm{CBF}$ values, the most important is the hematocrit (Hct) which is used to convert the arterial concentration of Xenon $\mathrm{Ca}(t)=C \max \left(1-e^{(b t)}\right)$ in CT Hounsfield units according to the equation: $C a(t)=C_{C T}$ * $X e(t) *(1+1.1 H c t)$, where $C_{C T}$ is the $\mathrm{kVp}$ dependent conversion constant, $X e(t)$ is end tidal Xenon measurement. Hematocrit measurement should be accurate, especially in acute unstable patients, because an inaccurately high Hct value is associated with the calculation of proportionally lower CBF values (Fig. 3).

\section{Specific Potential of Xenon-CT}

Lambda: Xenon is an ideal tracer for the measurement of CBF because it diffuses rapidly into the brain due to its high lipid content. This movement depends only on the volume of blood flow and the solubility of Xenon within the different tissue compartments of the brain. As lipid content is higher in white matter than in gray matter, solubility coefficient (lambda) is 1.4 in the former and 0.7 in the latter [17]. XeCT CBF is the only CBF technology that calculates lambda and integrates variations of this variable into the flow calculation. Therefore, this method most likely provides more accurate measurements than other quantitative CBF techniques even in disease conditions in which the lipid content could be altered. Fig. (4) shows differences in lambda occurring in apparently normal thalamus, in apparently normal frontal white matter and in a post-operative traumatic infarction. 


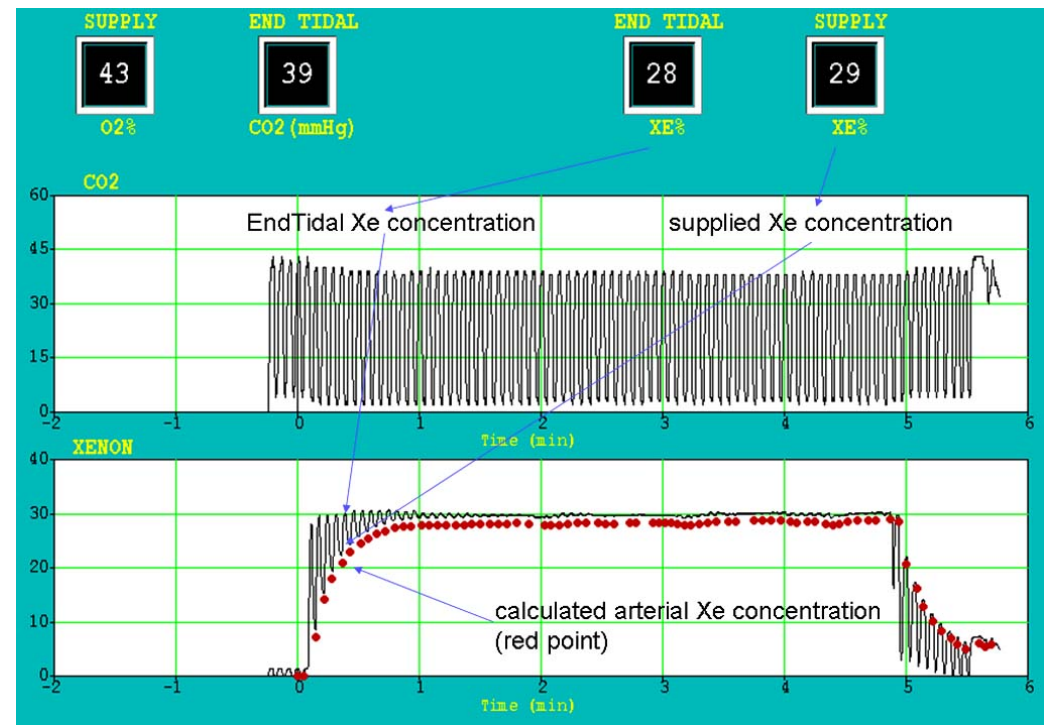

Fig. (2). The wash-in phase. After the start of Xenon inhalation, along the upstream increase in airways Xenon concentration, the oscillation in the Xenon curve are due to the progressive improvement in the equilibrium between inhaled and exhaled (end tidal) Xenon concentration. The red points indicate the estimated arterial Xenon concentration.

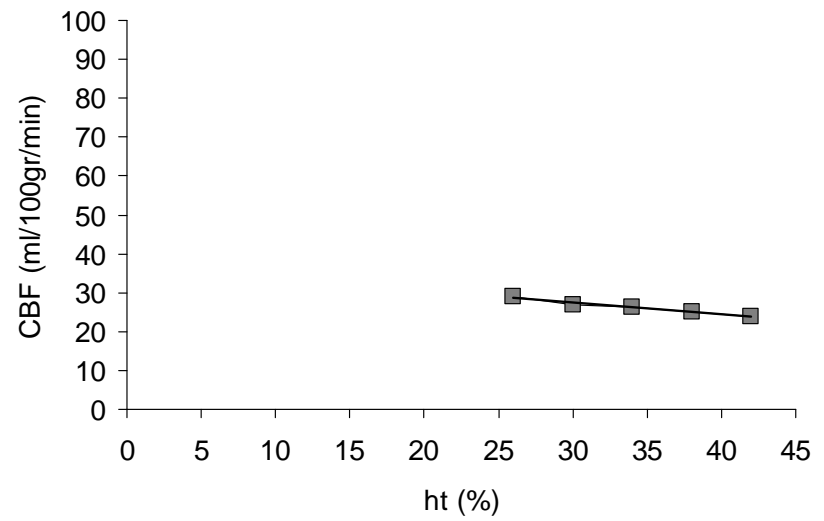

Fig. (3). In patient with an hematocrit of $34 \%$, we plotted the computation effect of simulated changes of Hct. An increase in hematocrit is associated with a decline in $\mathrm{CBF}$.
Double tests: the rapid wash out of Xenon through alveolar ventilation ( $\mathrm{t} / 230$ seconds) allows a fast clearance so that arterial Xenon concentration is less than $1 \%$ after 5 minutes. This fast xenon clearance allows repeated tests within an interval as short as 10-20 minutes from the baseline study.

\section{Limitations of Xenon-CT}

Bone: the partial volume effect related to bone may create artifacts, in particular, in basal frontal areas, (Fig. 5). lus.

Air: A similar problem may be due to pneumoencepha-

Metal: Artifacts due to metal affect Xe-CT more than CT image (Fig. 6). Care must be taken to remove any source of metal before the study.

Motion: Xe-CT studies in severe and moderate TBI patients who are artificially ventilated and sedated have potential advantages from immobilization. Movements on the part

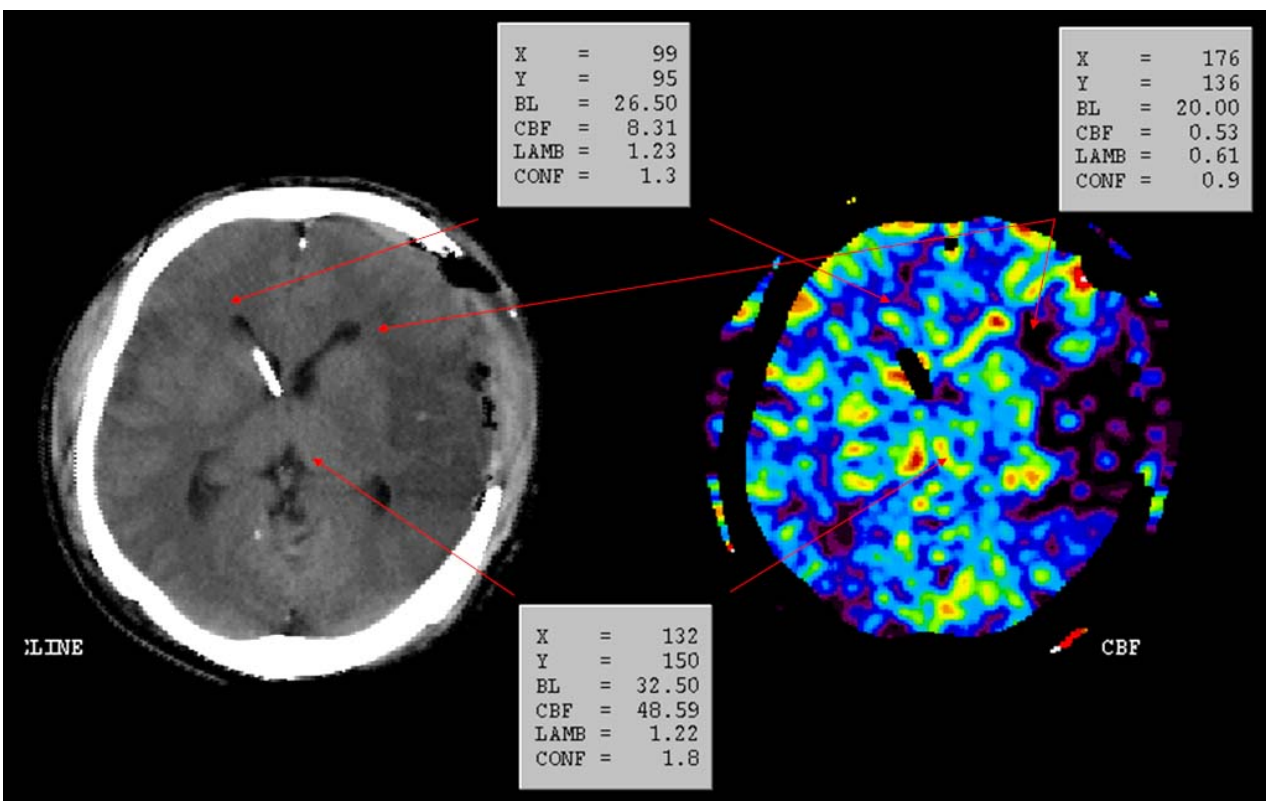

Fig. (4). Figure shows differences in lambda (LAMB) in the exemplificative pixels located in high flow cortical region (left thalamus), in low flow area (right frontal white matter) and in a post surgical traumatic infarction. 


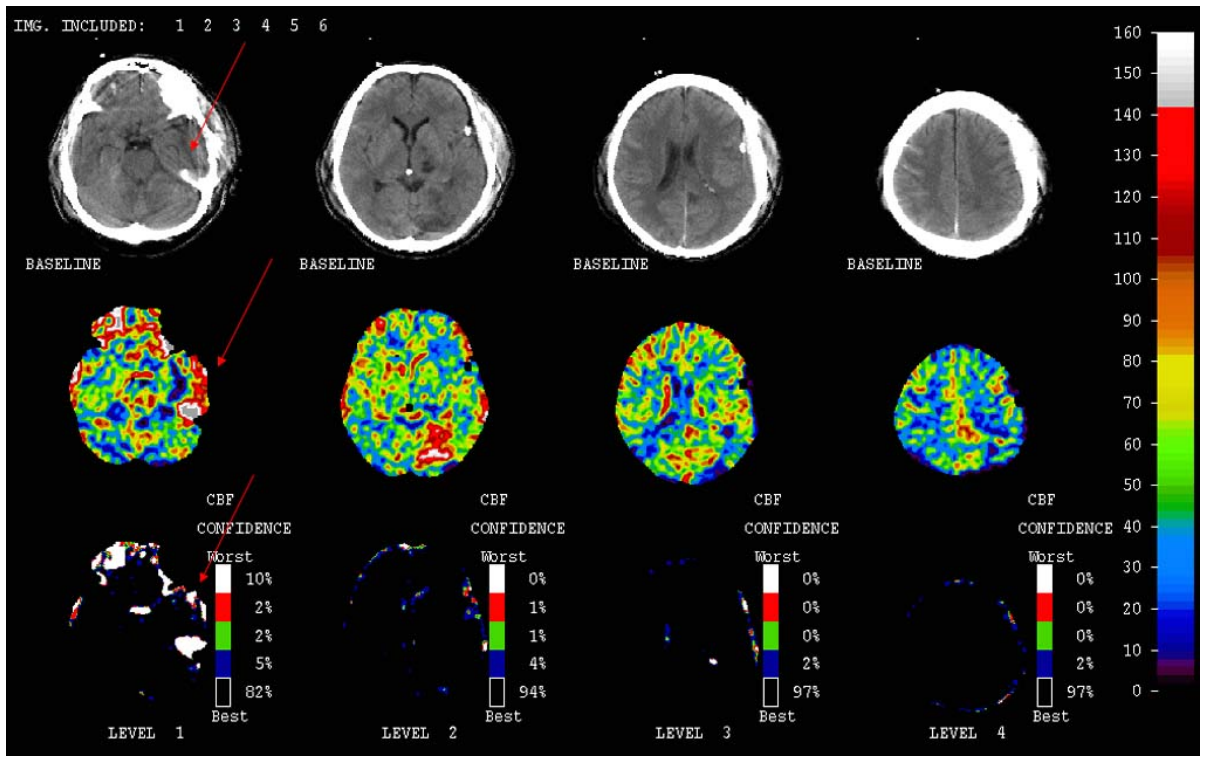

Fig. (5). In the basofrontal level and the left frontal lobe, on the left side artificial high rCBF values can be appreciated (arrows). The regional confidence analysis (last row) gives information on the reliability of CBF data. The artifact is due to the partial volume effect of the bone.

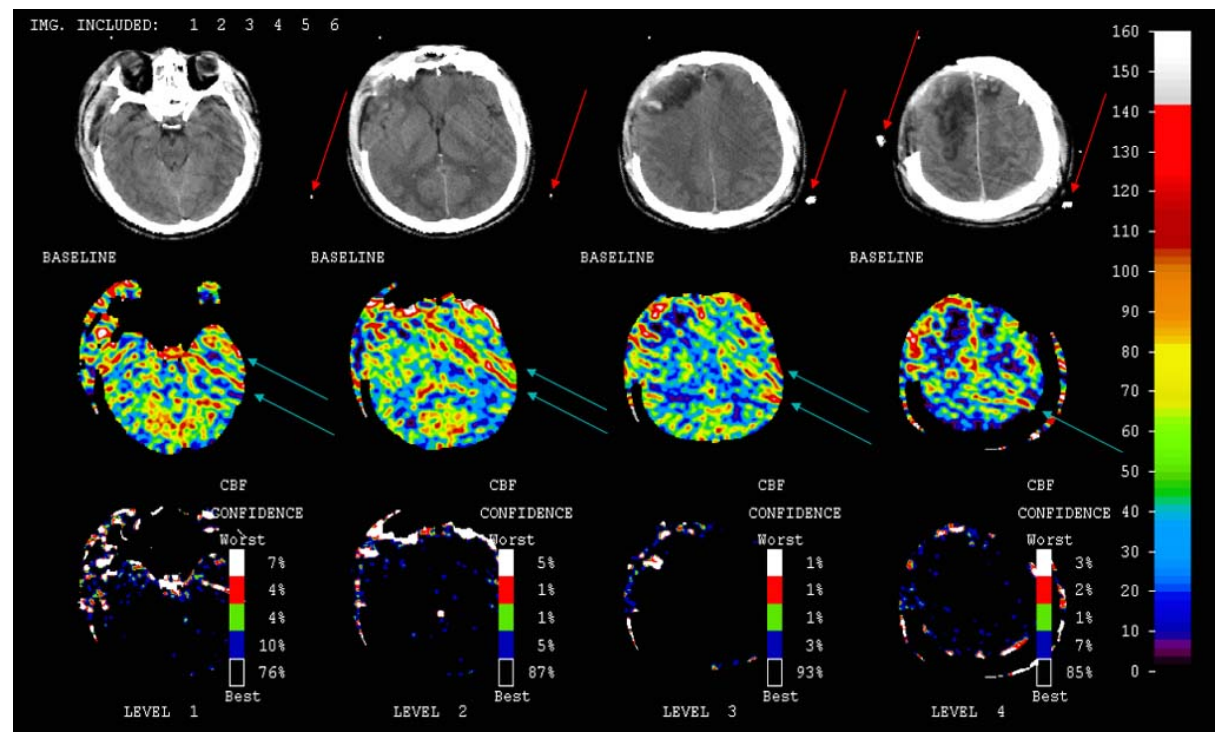

Fig. (6). Metal outside the brain (green arrows) generates oblique bands of artifactual hyperemia (red arrow).

of the patient may affect the alignment between the two baseline and the six inhalation scans. Consequently, motion mismatches the pixels among scans and the comparative calculation of the Hounsfield unit changes among the baseline pixel values toward inhalation pixel values (Fig. 7). An algorithm of the software (Xe-CT System Version $1.0 \mathrm{w}$ (C), 1998, Diversified Diagnostic Products, Inc, Houston, TX) measures such phenomena and alerts the reader by introducing threshold values. Conventionally, a motion greater than $15 \%$ is considered to be unacceptable. At times in TBI patients, a passive mobilization occurs between the baseline and the six wash-in period and this error makes the study impossible to recover. Conversely, movement of restless patients during wash-in, can be, in part, corrected by the XeCT software, by excluding the moved scans only [18].

Pulmonary mismatch: Among the basic assumptions for $\mathrm{CBF}$ calculation is the fact that the arterial xenon concentration curve can be derived noninvasively from measurements of the xenon end-tidal concentration. Although this statement is accurate with normal pulmonary function, giving a $\mathrm{rCBF}$ discrepancy of only $0 \pm 6 \%[19,20]$, there is evidence of a significant divergence between end-tidal and arterial xenon concentrations in patients with TBI, who often have multiple causes of severe ventilation/perfusion ratio abnormalities. As a consequence, $\mathrm{CBF}$ can be underestimated on average by $18 \%$ when a 8 -min wash-in, 8-min wash-out continuous xenon inhalation protocol is used [21]. A mismatch may be suspected if the slope to equilibrium between end tidal xenon and inhaled xenon is prolonged or the delta among the two values remains at plateau (Fig. 8).

Transport: In most hospitals CT scanners are not in the proximity of the ICU. It is well known that intra-hospital transport may be associated to secondary damage [22], which per se is a limitation for every extra ICU diagnosis. Modern centers currently include imaging units closely located to the ICU [23]. In patients with elevated ICP, who 


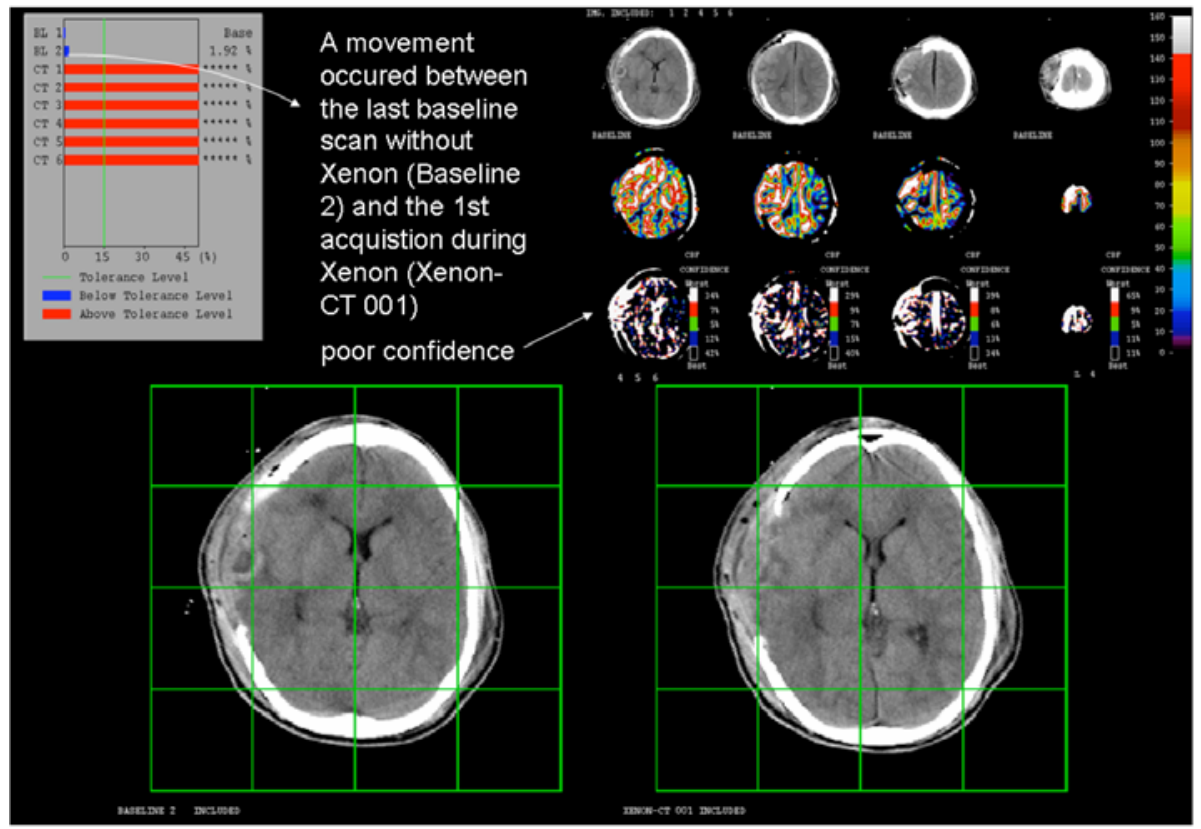

Fig. (7). A small change in the position of the head between the last baseline and the first wash-in CT scan explains the motion above the threshold value of $15 \%$ (red bars). The green grid helps the visual detection of the undue movement of the head. The poor quality of the study is signalled by the low confidence levels.

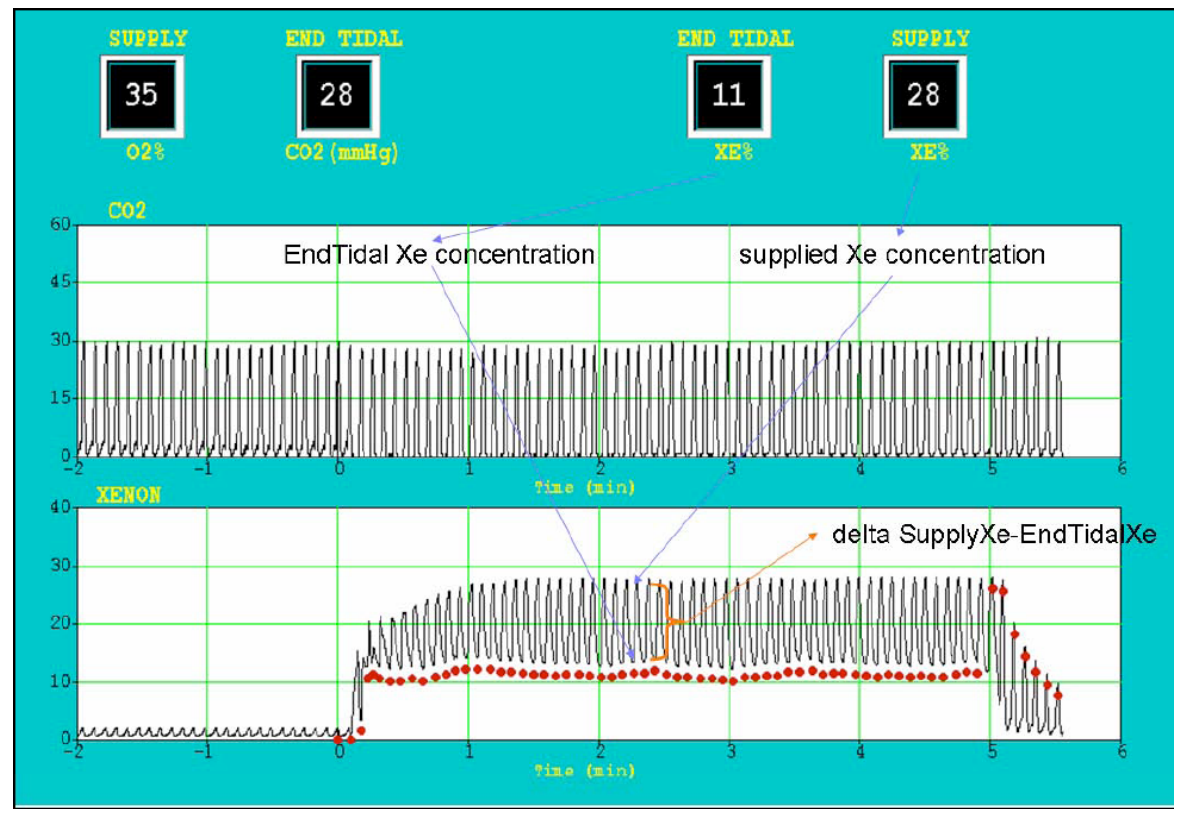

Fig. (8). End tidal Xenon never approached to inhaled ones. A severe impairment of gas exchanges should be suspected.

could greatly benefit from functional CBF measurement, clinicians must balance the risk of transportation with the advantage expected from the study. Furthermore, in the specific case of $\mathrm{CBF}$ measurement, even the most appropriate and careful transportation is unlikely to maintain physiological stability as in NeuroICU so that related CBF changes should be expected, which affects the reliability of measurement.

Radiation: Radiation is a limitation of Xe-CT, however, estimation of biological consequences is difficult to evaluate. A study from Seifert [24] calculated that exposure may be associated with one case of fatal cancer out of 12,500 studies. This risk is relatively uncertain. A further study esti- mated that out of 100,000 patients examined over a period of 25 years, only 4 developed brain tumors [25]. In our department the CT-scanner is set to administer $120 \mathrm{kv}$ and 150 mAs during the 32 slices of a Xe-CT study ( 8 basal slices and the 24 slices during the Xenon inhalation). The average surface dose per study is roughly of $400 \mathrm{mGy} / \mathrm{cm}$.

Flow activation: Although biologically inert and, therefore, ideal for CBF measurements, xenon induces an uncoupling of brain function and metabolism [20], resulting in an increase in $\mathrm{CBF}$. These findings have been accurately reviewed by Holl [26]. Analysis of the flow activation curve in humans with various intracranial insults showed a logarithmic shape [27]. An increase in TD-rCBF was observed be- 


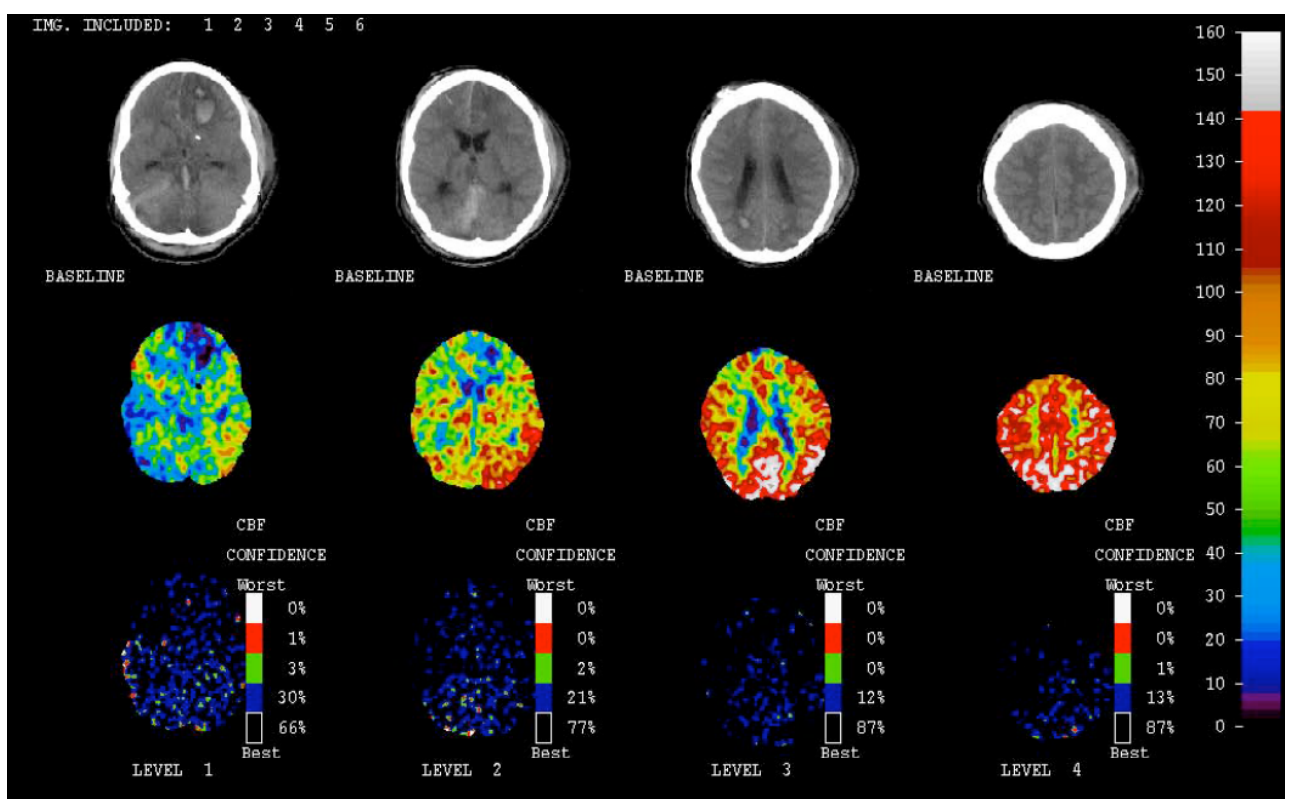

Fig. (9). During the planning of the wash-in phase there was an error in the scheduled acquisition of CT levels: the four levels were acquired rapidly six times in timely separated periods from each other by roughly 60 seconds. Consequently the levels were acquired at different concentration of arterial xenon. As a consequence, the last levels were most affected by flow activation. The consequences are falsely hyperemic values in these levels.

tween $3 \%$ and $7 \%$ within the first 76 seconds of Xenon wash-in (12\% after 190 seconds) while no further augmentation was detected until the end of the blood flow study [27]. A similar time course has recently been shown by Kim [28].

Fig. (9) illustrates a case in which flow activation is appreciable due to an error in the planning of scanning acquisition during the wash-in phase. All six acquisitions for the first basal skull level were acquired in rapid sequence, within seconds, followed after roughly 60 seconds by the next level. Owing to such imperfection, each level was acquired with a scheduled delay from each other. Consequently, as the basal slice was first acquired during the first minute after Xenon inhalation, it was the least flow activated, while, thereafter, the CBF increase seen in further levels was probably due to flow activation.

White matter: The low $\mathrm{CBF}$ in white matter prolongs the time needed to obtain an equilibrium between brain tissue and arterial xenon concentration. It has been estimated that more than 20 minutes are required to allow an accurate estimation of the lambda in white matter [29]. To reduce the risk of flow activation, however, the current wash-in phase lasts no longer than 4.5 minutes Xenon inhalation. Even if computational factor minimizes the relevance of such limitation on CBF calculation [14], the current method might underestimate $\mathrm{rCBF}$ values in white matter.

Areas at low flow states: Similarly to white matter, rCBF values might be underestimated in pathological areas affected by low flow values. These areas would be better evaluated with longer inhalation times [29].

\section{Potential Complications}

Increase in ICP: Xenon has an arterial vasodilatation effect, which is, in part, minimized by a low concentration $(28 \%)$ and by a reduced time to exposition (4.5 minutes, in the wash-in protocol). Xenon-induced dilatation produces an increase in cerebral blood volume (CBV) that can potentially result in ICP elevation, depending on the state of cerebral compliance [26]. Clinical studies have shown minimal changes in ICP [30,31]. In our experience most instances of ICP increase were related to transport, the loss of head elevation [32] and the maneuvers directed to increase the minute ventilation required to compensate the dead space of the xenon enhancer. In a subset of $370 \mathrm{Xe}-\mathrm{CT}$ studies (unpublished data) performed in TBI patients with ICP monitoring, our data showed a statistically significant higher ICP levels measured at the end of the Xe-CT study in comparison with ICP values obtained before the xenon inhalation. However, this worsening of ICP values appeared to be only marginally relevant from a clinical point of view (Fig. 10).

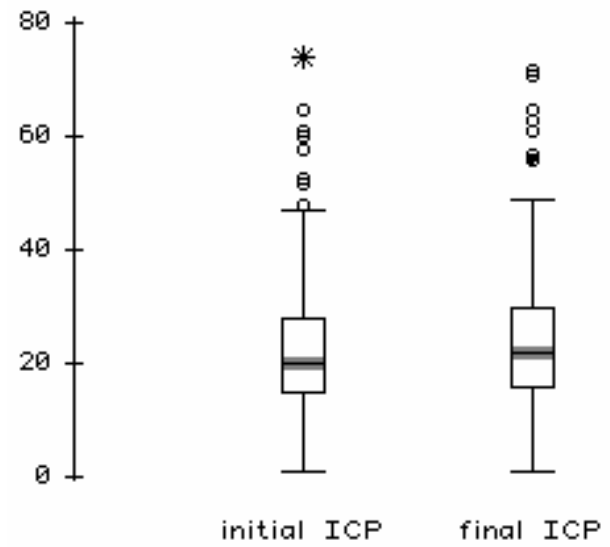

Fig. (10). Intracranial pressure (ICP) measured in $370 \mathrm{Xe}-\mathrm{CT}$ studies, before xenon administration and at the end of the Xe-CT study. Grey rectangles represent the $95 \%$ confidence interval for comparing median values.

Hypercapnia: The $28 \%$ xenon mixture (with oxygen and nitrogen) is delivered by an enhancer which is connected to 


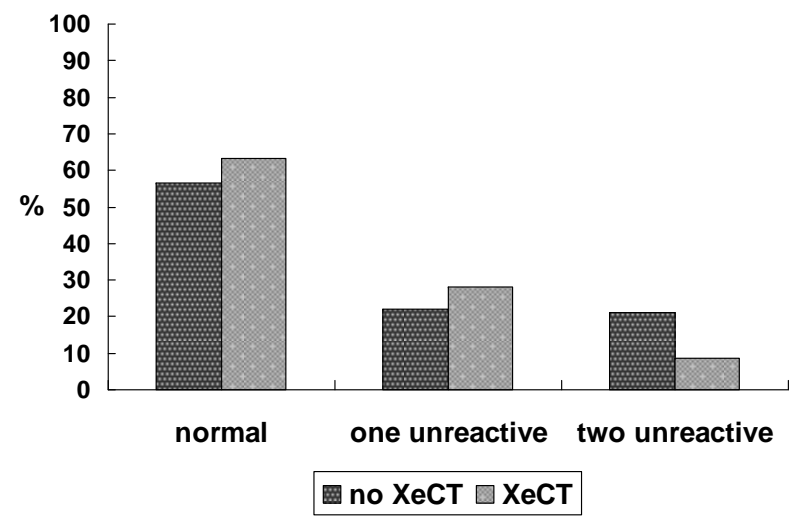

Fig. (11). "Severe" TBI patients submitted to at least one Xe-CT study presented less frequently with abnormal pupil reactivity to light at admission to neurosurgical hospital compared to those who were not submitted. Data on 625 patients consecutively admitted (unpublished data).

the mechanical ventilator. This enhancer has an internal dead space which must be added to the baseline tidal volume $(\mathrm{Vt})$ set in the mechanical ventilator. During the initial phase of connection to the enhancer, $\mathrm{EtCO}_{2}$ must be accurately checked, and should a value be above the baseline, Vt has to be increased accordingly. The lower the initial Vt, the more difficult it will be to find an appropriate setting. In such a context, pediatric patients deserve special attention. Inappropriate care or intrinsic difficulties in this phase may induce an acute increase in $\mathrm{EtCO}_{2}$ and, subsequently, in ICP. At times, a decline in $\mathrm{Vt}$ is associated to a decrease in pulse oxygen saturation.

Hypoxia: Patients with compromised pulmonary function requiring either a high level of pulmonary end expiratory pressure $\left(\geq 10 \mathrm{~cm} \mathrm{H} \mathrm{H}_{2} \mathrm{O}\right.$ ) and/or $\mathrm{FiO}_{2}$ values higher than 0.5 , may encounter difficulties during the Xe-CT once connected to the enhancer [33]. Indeed, $\mathrm{FiO}_{2}$ higher than 0.6 cannot be obtained due to the limiting effect in the gas mixture within the bag of the $28 \%$ xenon concentration.

\section{When, Why and How an Xe-CT Study Should be Per- formed?}

In terms of clinical practice, the nature of Xe-CT should be clear to the physician before attempting to use it. Is it a diagnostic tool, a physiological assessment, a monitoring modality or a test to dynamically evaluate the response to physiological or pharmacological challenges by means of repeated $\mathrm{Xe}-\mathrm{CT}$ tests?

Xe-CT imaging is a snapshot in nature and, consequently, may be considered a diagnostic tool [34,35] which allows us to answer the question of whether a disease or a physiological derangement is present or not. Therefore, threshold values for ischemia (or hyperemia) should be selected and validated, with their positive and negative predictive values (PPV and NPV). Consequently, we can hypothesize that Xe-CT study may lead to the diagnosis of a specific derangement guiding us to choose the therapy required to revert it. Unfortunately, only few studies have cross evaluated $\mathrm{CBF}$ towards an in vivo gold standard of ischemia, which at the present can only be morphological. Von Oettingen [36] showed that central low CBF areas in traumatic contusion evolve toward atrophy, but no cut-off value was evaluated. Similarly, no threshold values have been found for early global CBF when ventricle size (as a reflection of post-traumatic atrophy) is used as a late outcome measure of ischemia [37]. Most studies have assessed the relationship between ultra early ( $\leq 4$ hours) low global CBF values below $20 \mathrm{ml} / 100 \mathrm{gr} / \mathrm{min}$ and poor outcome [37], or the association between early post traumatic death and low CBF values, below $18 \mathrm{ml} / 100 \mathrm{gr} / \mathrm{min}$ [38]. More recently, a highly relevant paper by Cunningham [4] assessed CBF measurement (by means of PET, instead of Xe-CT) according to a probabilistic approach. The study showed that in TBI patients the PPV of threshold values for ischemia and penumbra is low. This result was confirmed by our team in patients with poor grade and complicated subarachnoid hemorrhage (SAH) due to a ruptured aneurysm, who were not selected for their a priori risk of post-procedural ischemia or vasospasm [39]. The most important cause of the poor PPV of CBF for ischemia is most likely to be the snapshot nature of $\mathrm{Xe}-\mathrm{CT}$. The time frame in which ischemia occurs is probably lost with episodic measurements. The second limitation of CBF measurements is represented by a reduction in oxygen metabolism developing independently after TBI $[40,41]$ or in association with sedative-induced metabolic depression [42]. The counfounding effect of reduced $\mathrm{CMRO} 2$ is probably associated with a decline, and more importantly, a variability of CBF threshold diagnostic for ischemia. These methodological limitations of CBF studies in diagnosing ischemia were correctly exposed by the Latronico group in 2009 [43].

Considering hyperemia, our recent paper [44] showed an association between focal cortical hyperemia beneath an evacuated subdural hematoma (SDH) and unfavourable outcome, without definition of the predictive values of threshold levels of hyperemia toward focal atrophy at CT. Similarly, an association, but not a prediction, between global hyperemia and unfavourable outcome was established by Kelly $[45,46]$.

So far, all these findings do not further support the categorization of Xe-CT within the range of diagnostic tools for TBI, but more likely suggest its potential as a physiological photography of global or regional derangements of $\mathrm{CBF}$. While thresholds of ischemia as outcome prediction of a tissue have only probabilistic values, classical threshold values may still be useful in providing a simplified, standardized and comparable CBF interpretation. Low CBF values (as a proxy of a risk or actual ischemia) have been derived from laboratory studies on the baboon [47-49] and from human studies on stroke [50]. For hyperemia (an excessive CBF compared to actual needs), a CBF threshold of 55.3 $\mathrm{ml} / 100 \mathrm{gr} / \mathrm{min}$ has been derived from the normal CBF distribution [51]. Furthermore, in comatose patients, cerebral metabolic rate of oxygen $\left(\mathrm{CMRO}_{2}\right)$ is expected to be physiologically reduced and, consequently, a coupled reduction in $\mathrm{CBF}$ can be anticipated [52] with preservation of normal arteriovenous oxygen content difference $\left(\mathrm{AVDO}_{2}\right)$. According to Obrist [51], any excess in $\mathrm{CBF}$, albeit within normal range, should be considered as a "relative" hyperemia $(\mathrm{CBF} \geq$ 33.9 and $<55.3$ ), while values abnormally high, according to the Gaussian distribution of CBF, can been defined as "absolute hyperemia" [51]. Thresholds vary among the various authors. In this setting, to allow comparison with previous studies, rCBF values have been classified according to the following already reported thresholds values [49-51]: $\mathrm{CBF}<6$ $\mathrm{ml} / 100 \mathrm{~g} / \mathrm{min}$ (severe ischemia), $\mathrm{CBF} \geq 6$ and $<18$ 


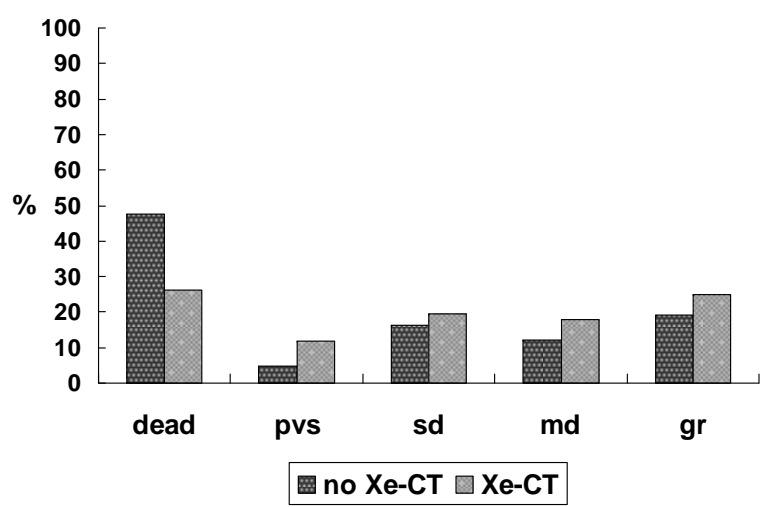

Fig. (12). Severe TBI patients submitted to at least one Xe-CT study are associated with better Glasgow Outcome Scale (GOS) [100] at one year post injury. Data on 625 patients (consecutively admitted unpublished data).

$\mathrm{ml} / 100 \mathrm{~g} / \mathrm{min}$ (moderate ischemia), $\quad \mathrm{CBF} \geq 18$ and $<33.9$ $\mathrm{ml} / 100 \mathrm{~g} / \mathrm{min}$ (reduced flow), $\mathrm{CBF} \geq 33.9$ and $<55.3 \mathrm{ml} / 100 \mathrm{~g} /$ min (relative hyperemia) and $\mathrm{CBF} \geq 55.3 \mathrm{ml} / 100 \mathrm{~g} / \mathrm{min}$ (absolute hyperemia).

A further potentially useful point of view is to look at $\mathrm{Xe}-\mathrm{CT}$ as a monitoring tool. A snapshot imaging is not, by definition, a monitoring, which in critical care medicine is a continuous observation of a parameter. However, in broader sense, repeated measurements of CBF by means of Xe-CT may give confirmation of the adequacy of management once adopted. For instance, at least three Xe-CT studies should be considered as an acceptable scheduled frequency of administration. Although this approach represented, until recently, the only possible approach to monitor $\mathrm{CBF}$, after many years of pre-clinical application, the quantitative measurement of rCBF by means of TD-CBF [7] is now available as a real monitoring, albeit focal, at bedside. Even in consideration of all doubts concerning physiological thresholds, a further reason to measure $\mathrm{CBF}$ is to use it as a reference, comparing $\mathrm{CBF}$ after short perturbation of physiological variables, the so-called Xe-CT double test. These variables are normally used to evaluate meaningful responses to therapies. To our knowledge, only four kinds of tests have been applied by means of Xe-CT in TBI patients: induced hypocapnia [5355], indomethacin bolus [56], norephinephrine-induced CPP elevation [57] and increase in $\mathrm{FiO}_{2}$ from 0.35 to 0.6 [6].

\section{Bias in Selection of Patients on Clinical Grounds}

As reported above, the patients who undergo Xe-CT are the result of a careful selection based on specific determinants. Since 2000 to date, only 234 out of 625 patients with severe TBI (37.4\%), consecutively admitted to our NeuroICU, have undergone at least one Xe-CT study. Particularly, severe patients with abnormal pupil response and wide mass lesions are not often evaluated. This explains why only three series reported ultra early CBF measurement [38,58, 59]. Consequently, patients at high risk of early global and focal ischemia and patients with refractory intracranial hypertension are often excluded, although CBF measurement may be more useful for them than in less severe patients. The reduced representation of patients with bilateral unreactive midriasis on admission (Fig. 11) and the high proportion of patients with better outcome among those who underwent Xe-CT confirm a case mix selection (Fig. 12).

\section{Physiological Findings During Xe-CT Study}

The interpretation of $\mathrm{CBF}$ values relies on the stability of physiological variables during the Xe-CT study. This is the specific objective of the physician, technicians or nurses under whose care the patients are placed. Furthermore, variables have to be recorded. The minimum mandatory set is: $1^{\text {st }} \mathrm{PaCO}_{2} ; 2^{\text {nd }}$ mean arterial pressure; $3^{\text {rd }} \mathrm{ICP}$, if available, and consequently $\mathrm{CPP} ; 4^{\text {th }}$ pulse oxygen saturation; $5^{\text {th }} \mathrm{FiO}_{2}$; $6^{\text {th }}$ temperature; $7^{\text {th }} \mathrm{Hb}$ and Hct, the latter mandatory for CBF algorithm calculation. Furthermore, when a retrograde jugular catheter is inserted, simultaneous sampling of arterial and jugular bulb venous blood [60] for blood gas analysis and lactate and glucose measurements, is used to calculate the difference between arterial and venous contents of oxygen $\left(\mathrm{AVDO}_{2}\right)$, and concentrations of glucose (AVDG) and lactate (AVDL). According to the Fick law, $\mathrm{CMRO}_{2}$, cerebral metabolic rate of glucose (CMRG) and of lactate (CMRL) can be measured by multiplying the global $\mathrm{CBF}$ value, obtained after the Xe-CT study, for the corresponding arteriovenous differences.

\section{Different Outputs Format of Xe-CT Study}

After the performance of an Xe-CT study we have, at least, five types of potential outputs: $1^{\text {st }}$, a colored map of CBF for each of the four CT levels examined (Fig. 13); $2^{\text {nd }}$, quantitative analysis of data by means of single or multiple Region of Interests (ROI) wider than at least $8 \times 8 \times 10 \mathrm{~mm}^{3}$ ( $8 \mathrm{~mm}$ circle), to keep the error within $20 \%$ for flow in the range of 20 to $80 \mathrm{ml} / 100 \mathrm{gr} / \mathrm{min}$ (Fig. 14) [61]; $3^{\text {rd }}$, quantitative analysis according to the vascular territory (Fig. 15) [62]; $4^{\text {th }}$, pixels analysis of CBF according to selected thresholds (Fig. 16); $5^{\text {th }}$, free hand ROIs outlined on selected CT lesions (Fig. 17).

The colored map, due to the quantitative measurement of $\mathrm{Xe}-\mathrm{CT}$, corresponds to a continuous quantitative scale, but, obviously, visual perception makes it semi-quantitative. Data analysis requires a little more time and experience with the software. Consequently, at least in our Department, the physicians involved in the clinical field try to obtain functional indications more from semi-quantitative (colored maps) than quantitative analysis which is mainly restricted to research field. Such visual examination of CBF would allow us to move on different levels according to the interest that may focus mainly on either global or regional CBF. If the interest was directed to regional $\mathrm{CBF}$, we would try to detect areas of low $\mathrm{rCBF}$ potentially leading to future brain damage. In this case, a low CBF should be considered as a risk factor, a potential cause of further damage (Fig. 18). Otherwise, we should focus our perspective more on the appearance of rCBF when the lesion has already been established. In such a case, the rCBF more likely represents the effect of previous pathogenetic mechanisms leading to the lesion rather than the actual cause of the lesion itself. That is the case of hyperemia in low density region (Fig. 19). This finding is typical of lesions due to reversible compression or distortion of major artery territories. On the other hand, the rCBF measured in correlation with a CT lesion may be a cause of a further evolution of the lesioned areas (Fig. 20). For example low $\mathrm{rCBF}$ value in the periphery of a traumatic contusion can be implicated in a further extension of edema. In addition, regional $\mathrm{CBF}$ in a injured area may be useful as a proxy of tissue viability. 


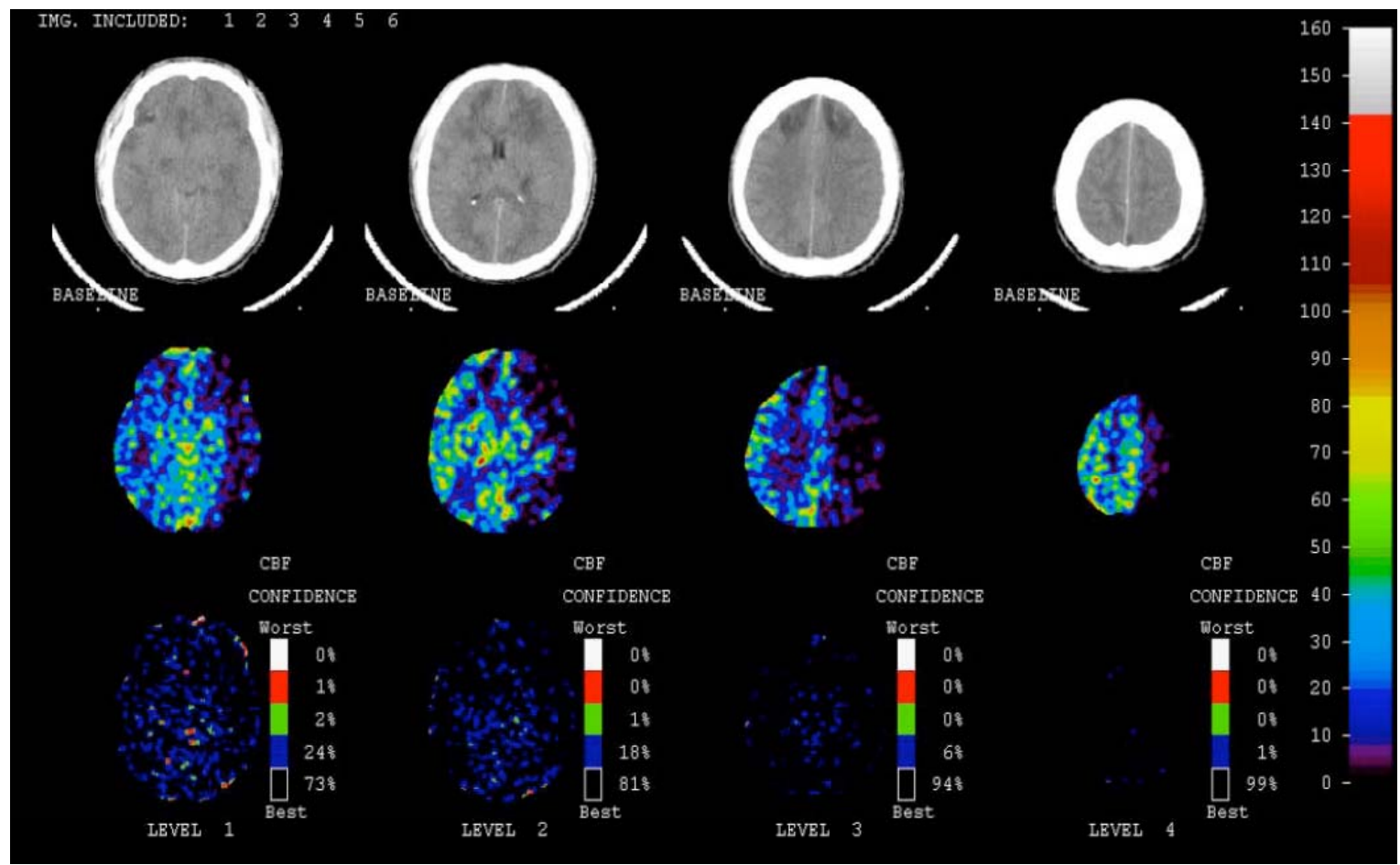

Fig. (13). The coloured map of rCBF in the four levels. This is the raw output of Xe-CT study.

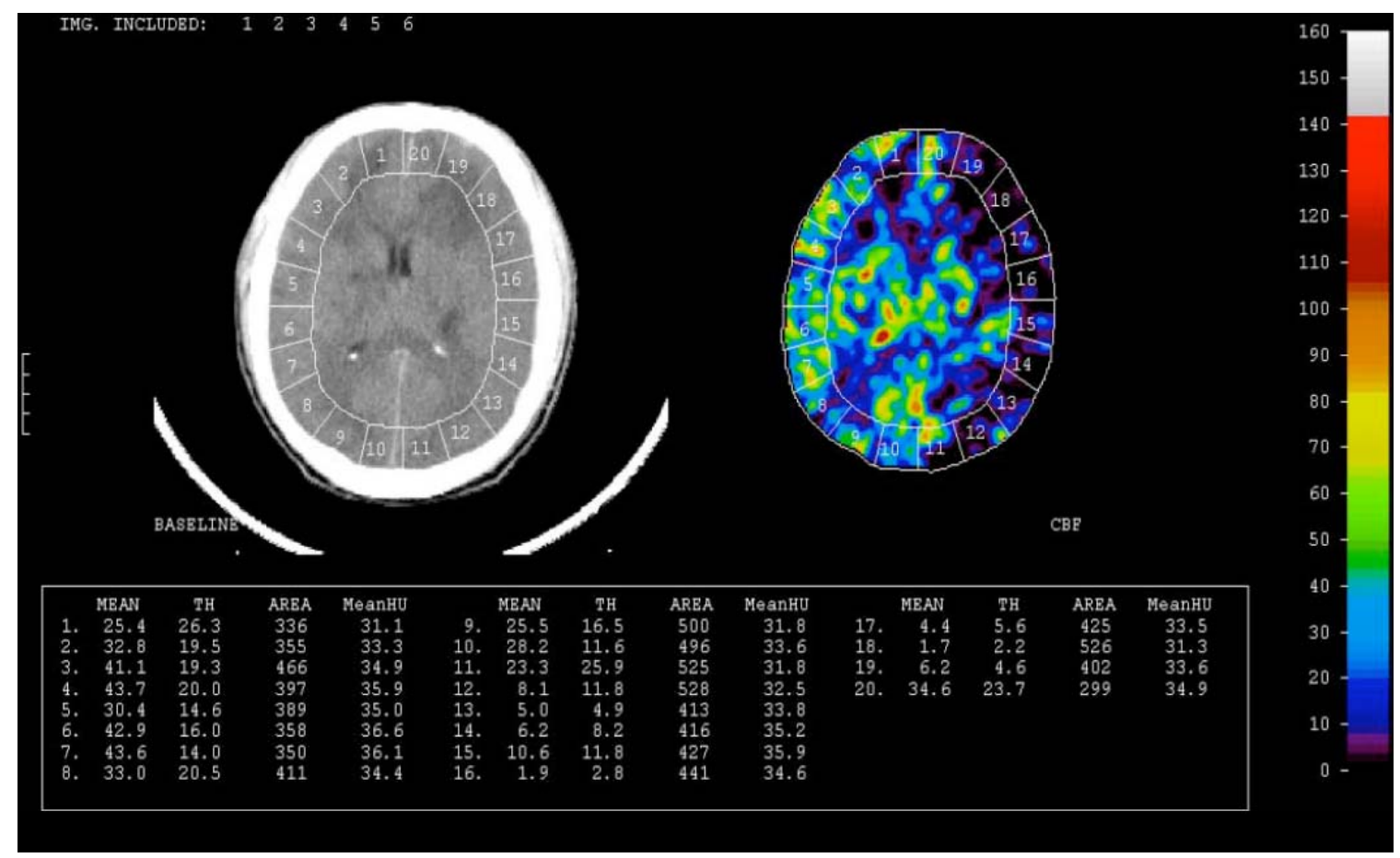

Fig. (14). The same Xe-CT study as in Fig. (13) with 20 regions of interest (ROI) placed on the cortex. Their area is wider than $300 \mathrm{~mm}^{2}$.

\section{Important Factors Affecting Xe-CT Interpretation}

Regardless of the selected output, CBF interpretation of a single study or repeated studies over days is affected by several factors, such as the natural history of disease, physiological changes in $\mathrm{CBF}$ or relevant variables, changes in management, etc. As all these variables are difficult to account for in a multivariate interpretation, this further reduces the chance of evaluating any causative effect of $\mathrm{CBF}$, supporting a more practical use of $\mathrm{CBF}$ as a monitoring tech- nique. However, at least four major findings always have to be considered: 1 - the difference in CBF levels in low and high flow areas such as gray and white matter contents; 2 the influence of age on global CBF; 3 - the spontaneous time course of $\mathrm{CBF} ; 4$ - the effect of sedative therapies.

\section{High and Low Flow Areas}

In the original paper from Yonas [63], it is clearly described how the brain can be simplified into low and high 


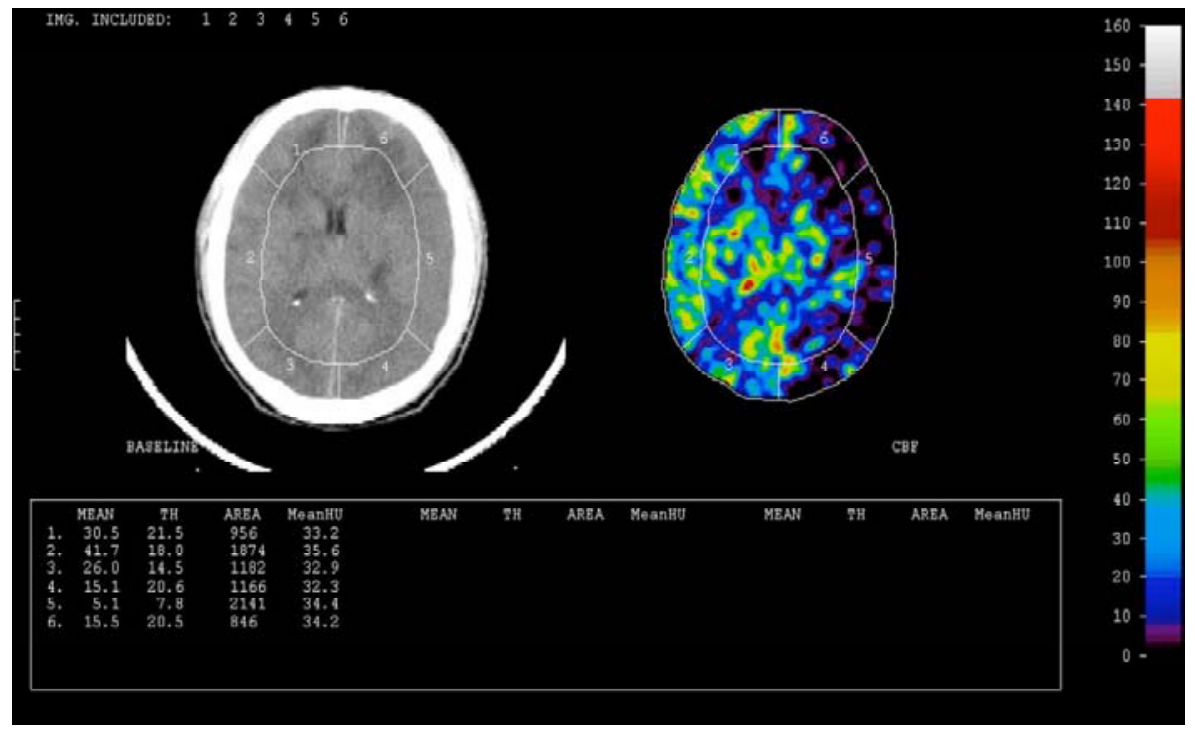

Fig. (15). The same Xe-CT study that in Figs. (13 and 14). The ROIs are now drawn according to main arterial vessels distribution, posterior cerebral artery, middle cerebral artery, anterior cerebral artery.

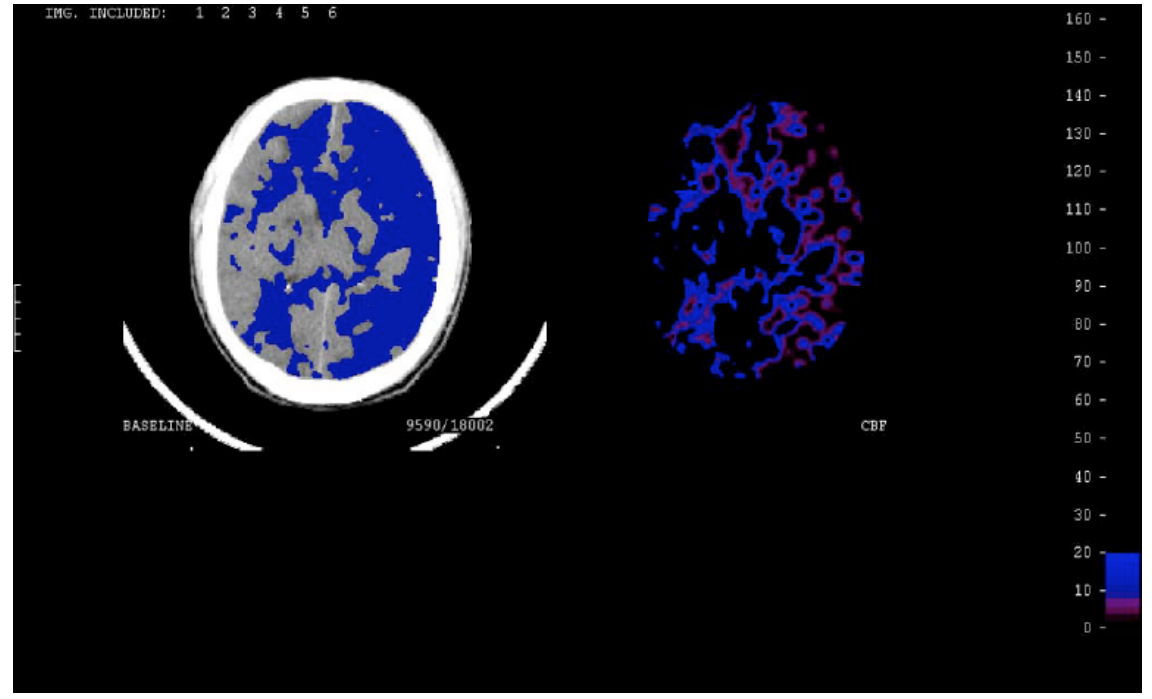

Fig. (16). The same Xe-CT study as in Figs. (13), (14) and (15). Pixels have been filtered according to a threshold value of CBF equal to 20 $\mathrm{ml} / 100 \mathrm{gr} / \mathrm{min}$. Only pixels with CBF values below $20 / \mathrm{ml} / \mathrm{min}$ are shown in CBF map and are superimposed on CT. The ratio of pixels below $20 \mathrm{ml} / 100 \mathrm{gr} / \mathrm{min}$ compared to total pixels slices are described (9590/18002).

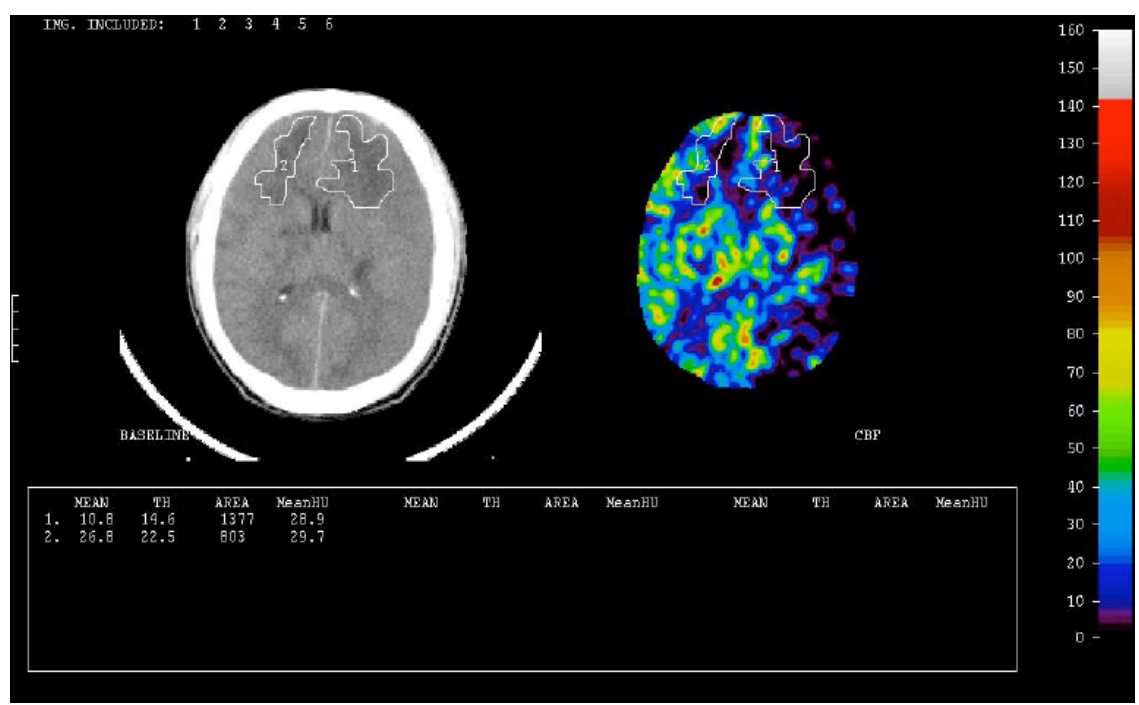

Fig. (17). The same Xe-CT study as in Figs. (13), (14), (15) and (16). Region of interest (ROI) drawn free hand on hypoattenuation areas. 

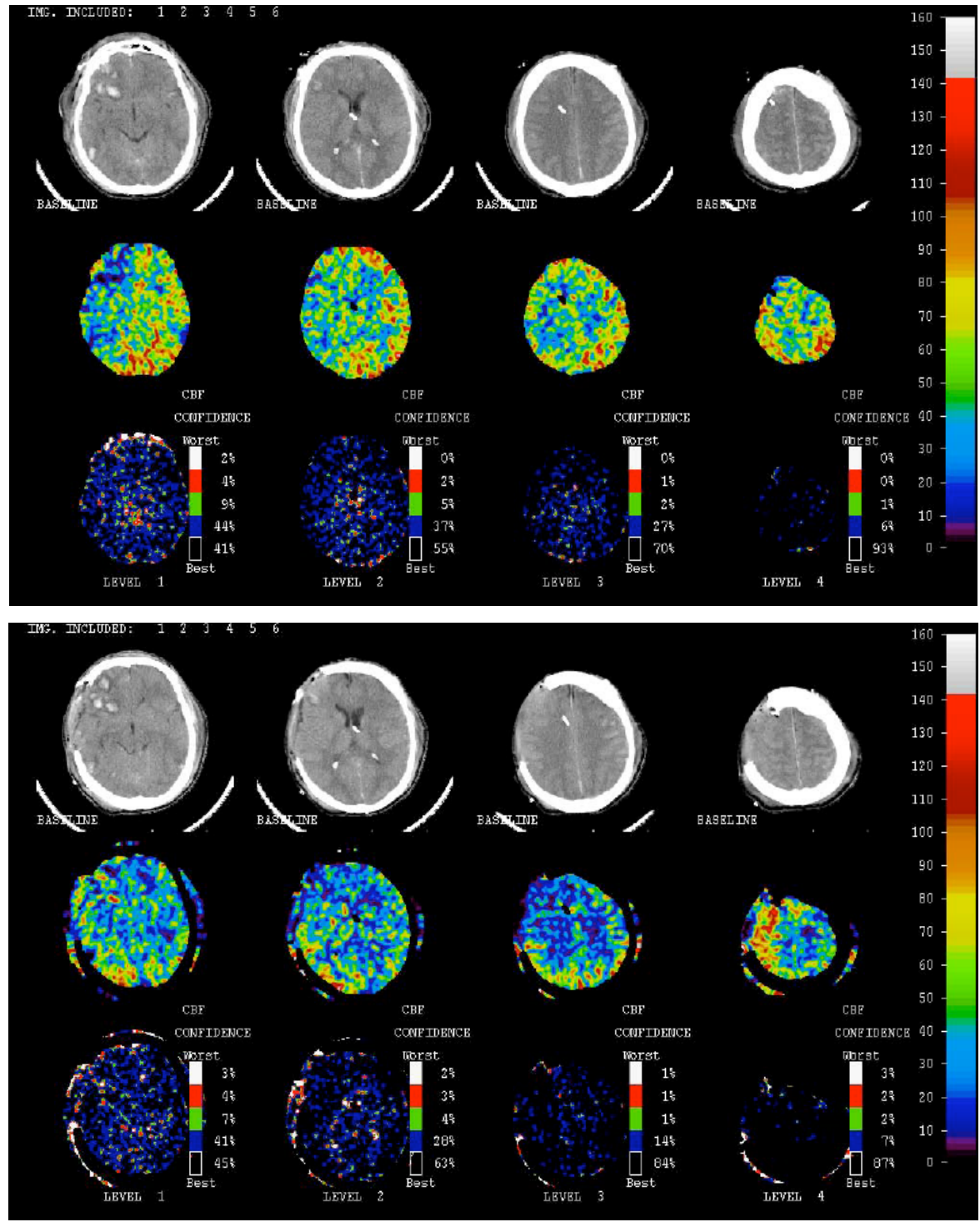

Fig. (18). Panel at the top: patient with a reduction in $\mathrm{rCBF}$ in right hemisphere probably in association with regional intracranial hypertension. Panel at the bottom: the same patient after external decompression: the asymmetry of rCBF between the two hemispheres observed in the first Xe-CT study is no longer perceived. In such case the reduction in rCBF observed in the first Xe-CT was a potential cause of regional damage, while external decompression relieved regional hypoperfusion.

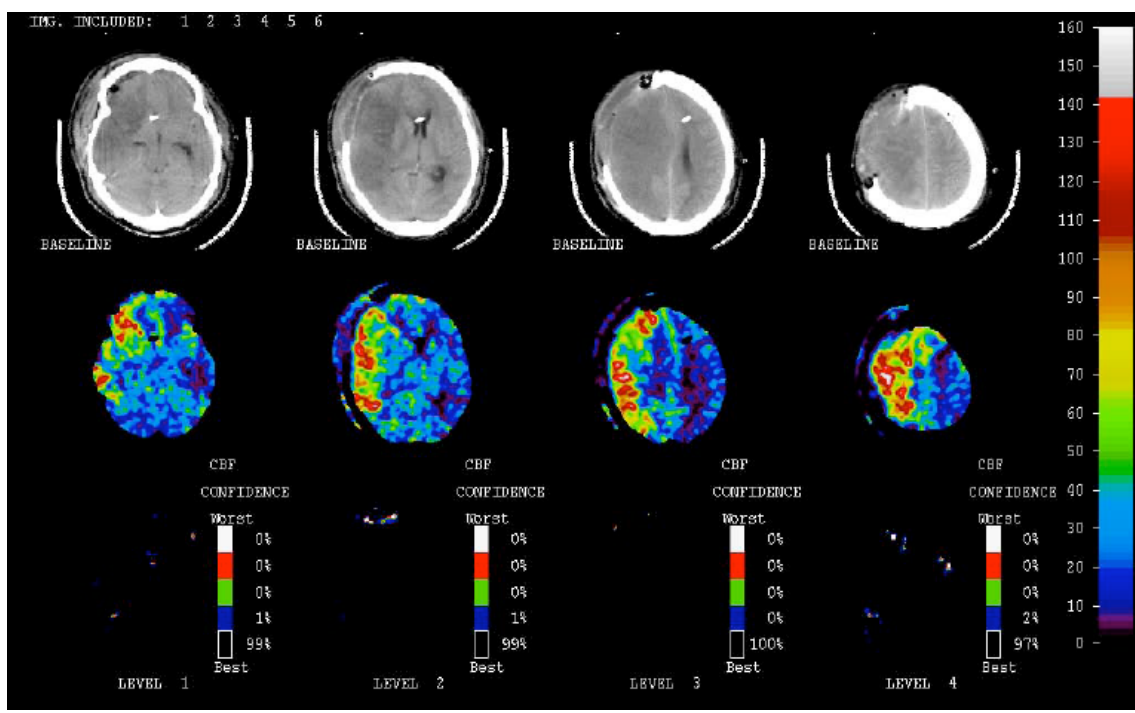

Fig. (19). Low attenuation in the area of the right middle cerebral artery due to a subacute sudural hematoma. After surgical removal, the hypoattenuation was found to be associated with hyperemia. In this case, hyperemia is probably an effect of a previous ischemia. 


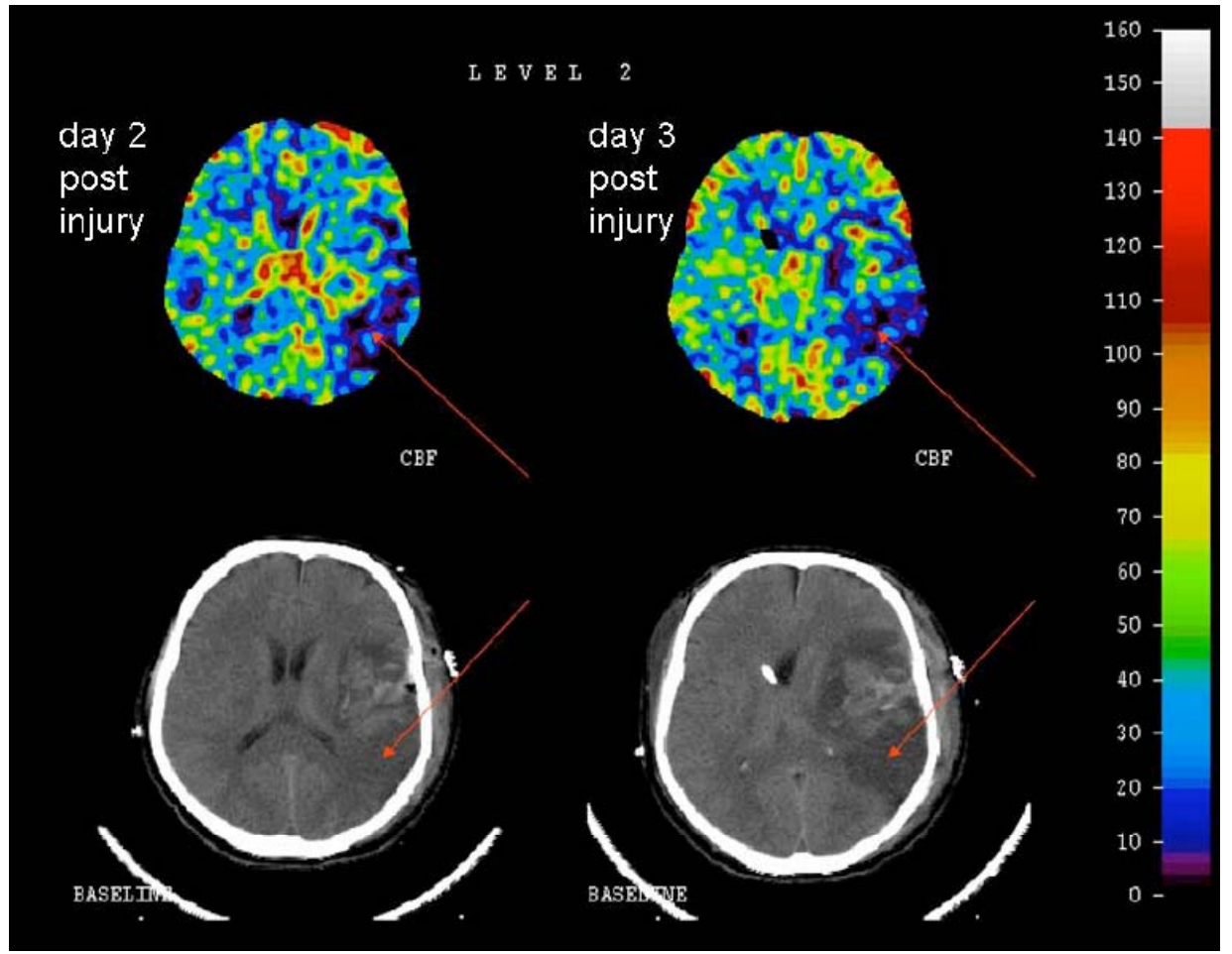

Fig. (20). $\mathrm{rCBF}$ decrease below a traumatic hematoma is associated to a worsening of hypoattenuation in the next Xe-CT study. The further reduction in $\mathrm{rCBF}$ is the cause of further damage.

flow compartments. In fact, during analysis, the ROIs should be greater than $200 \mathrm{~mm}$, to reduce the error of CBF measurement below $12 \%$ [16]. Consequently, the resolution of analysis is unable to discriminate between the cortical cortex and the underlying white matter. Areas with high flow are those with more gray matter: the cortical region, the thalamus and the basal ganglia. White matter, with the internal capsula is an area with low flow. The CBF in high and low flow compartments, sampled by placing 5-mm circular ROIs in regions containing the highest and lowest flow values in each hemisphere, averaged, in the original paper of Yonas [63], $84 \pm 14$ and $20 \pm 5 \mathrm{ml} / 100 \mathrm{gr} / \mathrm{min}$, respectively [63]. These normative values should be kept in mind and compared with traditional values of ischemia threshold for the cortex which are $18 \mathrm{ml} / 100 \mathrm{gr} / \mathrm{min}$ [49] and that for white matter, which should be at a much lower level (7 $\mathrm{ml} / 100 \mathrm{gr} / \mathrm{min}$ ) [64].

\section{The Influence of Age in Global CBF}

Cerebral blood flow is deeply influenced by age, especially during the pediatric period [65]. On the basis of the paper by Suzuki [66] in unanesthetized, normal children, $\mathrm{CBF}$ may range from $40 \mathrm{ml} / 100 \mathrm{gr} / \mathrm{min}$ during the first 6 months of life to $108 \mathrm{ml} / 100 \mathrm{gr} / \mathrm{min}$ at 3 to 4 years of age, and then it decreases to $71 \mathrm{ml} / 100 \mathrm{gr} / \mathrm{min}$ after 9 years of age [67]. After 19 years of age, CBF decreases gradually to adult levels. During ageing a further decline in CBF occurs [63], predominantly in the high flow compartment.

In TBI patients this association between ageing and reduction in $\mathrm{CBF}$ is depicted in Fig. (21), whereas raw values of global CBF per age are summarized in Fig. (22). The decline in $\mathrm{CBF}$ is probably a consequence of the physiological reduction in $\mathrm{CMRO}_{2}$ [68] and CMRG. From a practical point of view, interpretation of $\mathrm{Xe}-\mathrm{CT}$ in children should take into consideration that diffusely elevated rCBF values, specifically in cortical regions, are not automatically to be considered as pathological hyperemia. Consequently, interventions, e.g. indomethacin [69] or hyperventilation to control a physiological phenomenon are not justified. Furthermore, to our knowledge, age-adjusted CBF thresholds for ischemia are unknown, but they should be higher than in adults. Consequently, apparently adequate and decreased CBF values in children should be evaluated with caution.

Conversely, markedly reduced CBF values in elderly patients [70] must not automatically be considered as a risk for ischemia, but, for example, the relevance of added sedation should be taken into account. This latter aspect is particularly relevant when considering that the aged brain is more sensitive to the depressive effect of sedation, if the dose is not adjusted for age [71].

\section{Time Course of CBF}

Several studies have evaluated the time course of $\mathrm{CBF}$ after injury and converging findings suggest that, in the first hours post injury (phase 1), global CBF is markedly reduced, increases then during days 2-4 (phase 2) and thereafter decreases toward levels higher than those observed in the first 24 hours (phase 3), both in adults [72-76] and in children [77,78]. Fig. (23) describes the time course of global CBF in 522 studies (unpublished data). A similar time course of rCBF has been observed in traumatic contusion by means of intravenous ${ }^{133}$ Xenon [79], in traumatic intra-parenchymal hematoma [75] and in the cortex below the evacuated SDH by means of Xe-CT [44]. Although this pattern has been described in pooled patients with TBI it seems to characterize only patients with unfavorable outcome as shown in two 


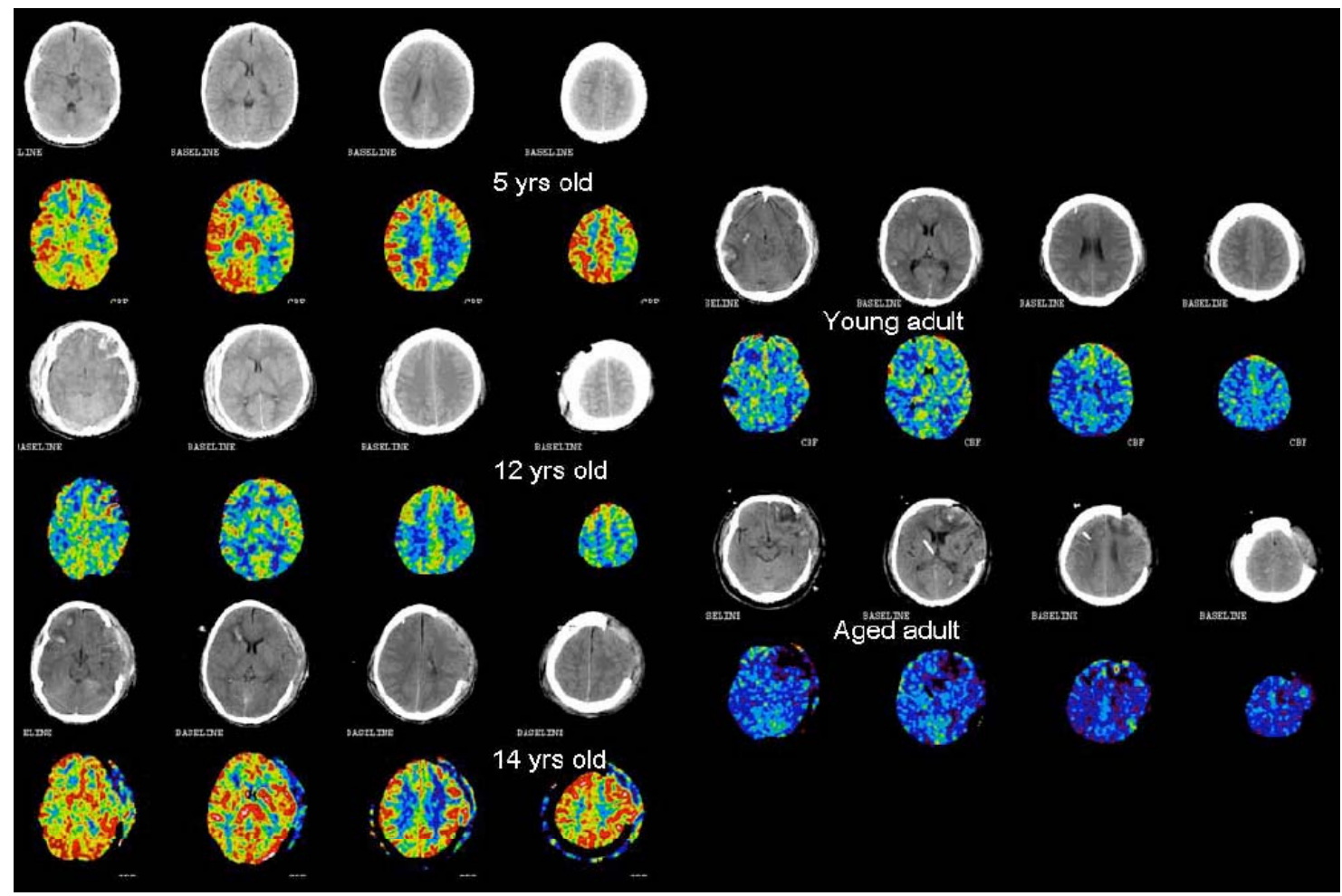

Fig. (21). Illustrative cases showing the decline in CBF with ageing.

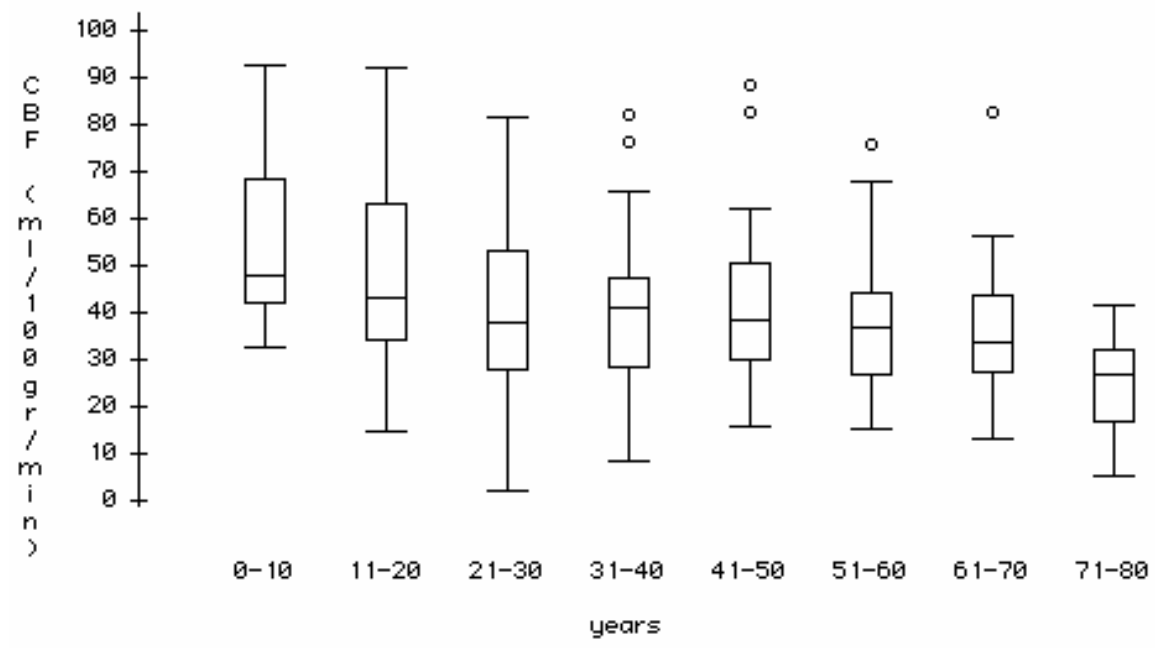

Fig. (22). Box plot showing median and interquartile ranges of global CBF according to age. Data from 500 Xe-CT studies (unpublished data).

studies in which data were dichotomized for favourable and unfavourable outcome $[44,76]$. According to these studies, this pattern proved to apply to both global CBF [76] and regional $\mathrm{CBF}$, at least in patients affected by isolated $\mathrm{SDH}$ [44].

Interpretation of the CBF time course is not yet clear, and several different factors may interact at the different phases of the time course. Indeed, initial CBF depression (phase 1, oligoemic-ischemic) may represent the result of several effects. First, the reason for early oligemia could be merely computational as all studies included patients who died early and in whom very low CBF values and irreversible ischemia were established [38,59]. Consequently, these patients, by self exclusion, did not contribute to the more elevated levels of $\mathrm{CBF}$ observed in phase 2. Second, immediately after trauma a non ischemic decline in $\mathrm{CMRO}_{2}$, coupled with subsequent $\mathrm{CBF}$ decrease, occurs as a result of physiological changes after the initial impact [80-83]. Third, several factors may contribute to true global, multifocal or regional hypoperfusion, without an association with an irreversible outcome. Recently, a study by the Robertson group [58] showed that an early reduction in global CBF is predominantly associated to elevated ICP levels and reduced CPP values. The hypothesis that low CBF values should be due to ischemia was also supported by PET studies [84, 85]. Consequently, today, it would seem that early low CBF values 


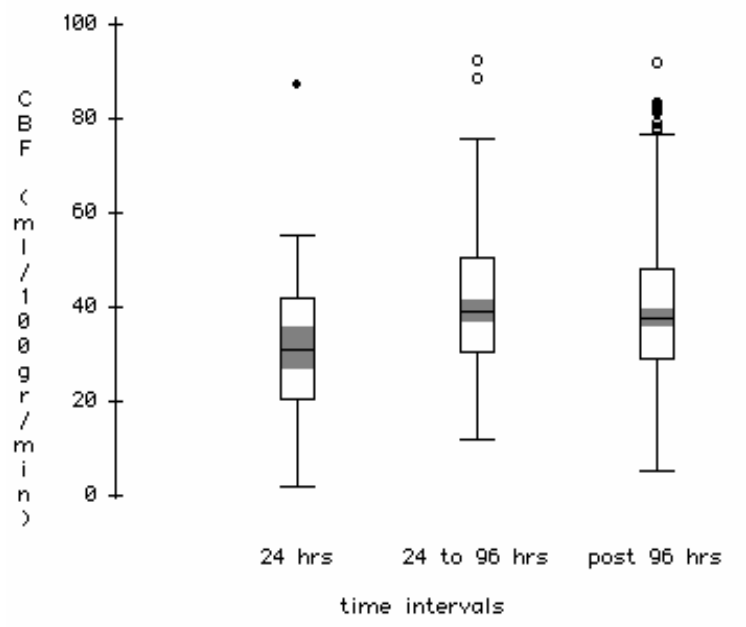

Fig. (23). Time course of global CBF in $522 \mathrm{Xe}-\mathrm{CT}$ studies (unpublished data). Grey rectangles represent the $95 \%$ confidence interval for comparing median values.

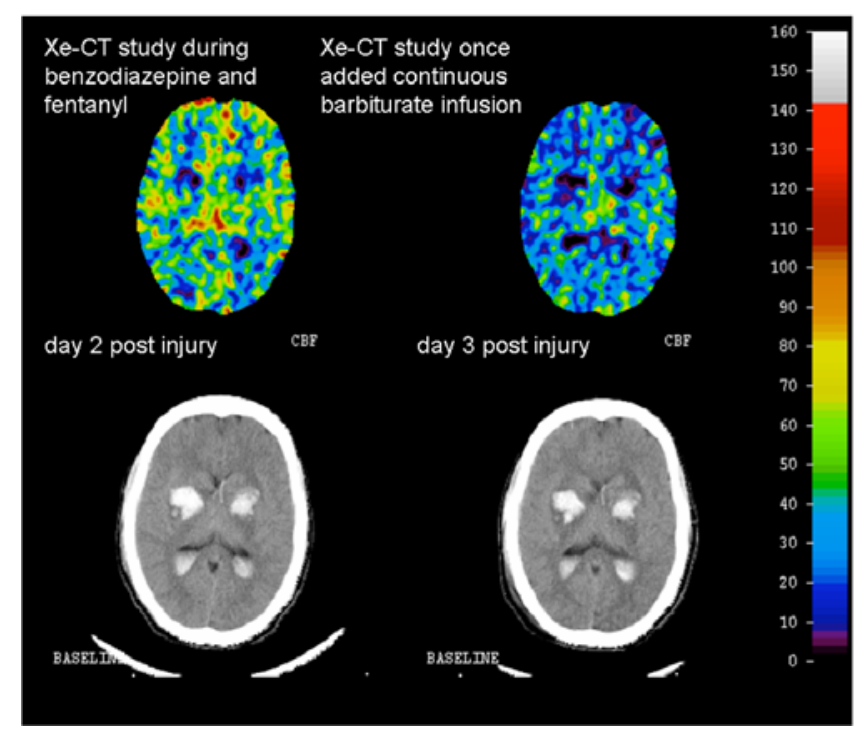

Fig. (24). A young adult patient with severe TBI and refractory intracranial hypertension. The more elevated baseline CBF was obtained during deep continuous sedation with benzodiazepine and fentanyl. However the day after, due to a progressive increase in ICP, continuous barbiturate infusion was added to previous sedation plane. The correspondent depressed global CBF should be the consequence of the barbiturate induced decline in cerebral metabolic rate of oxygen.

below $18 \mathrm{ml} / 100 \mathrm{gr} / \mathrm{min}$ could be a red flag for true ischemia. Similarly to phase I, phase 2 (hyperemic) may, in part due, be to the exclusion of patients with very low CBF who died and were not further observed. More probably, however, hyperemia represents a state of uncoupling between $\mathrm{CMRO}_{2}$ and $\mathrm{CBF}$ in favor of a coupling between CMRG and CBF [86]. An increase in CMRG is probably due to enhancement of glycolysis which is useful in reparative processes. Assuming that higher repair needs are associated with more severe initial damage, more hyperglycolysis can be expected, resulting in worsening of $\mathrm{CMRO}_{2} / \mathrm{CBF}$ uncoupling. Enhanced $\mathrm{CMRO}_{2} / \mathrm{CBF}$ uncoupling may, in turn, explain the association between unfavourable outcomes and global [76], as well as regional, [44] hyperemia. The third phase, with a reduc-

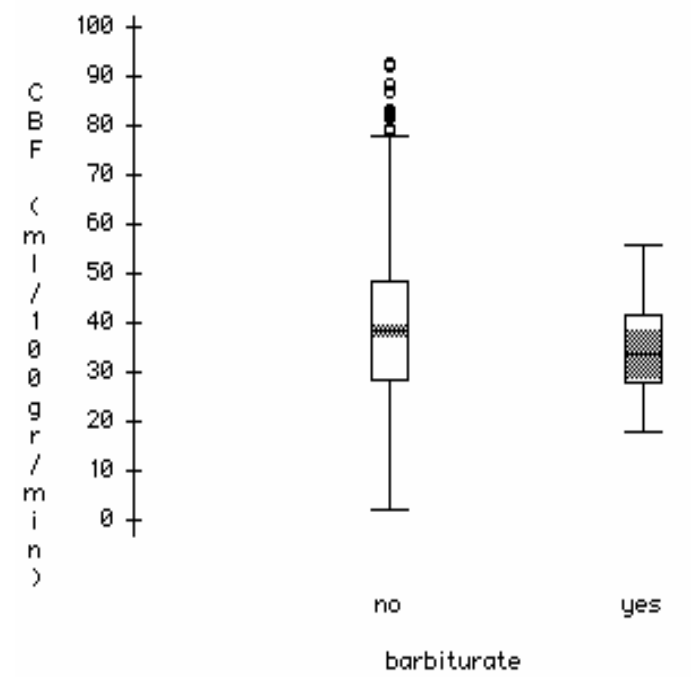

Fig. (25). Median global CBF values are lower in Xe-CT studies obtained during barbiturate infusion. Data from $522 \mathrm{Xe}-\mathrm{CT}$ studies (unpublished data) Grey rectangles represent the 95\% confidence interval for comparing median values.

tion in CBF to a moderate level, seems to be variable and due to a hypometabolic phase, spontaneously occurring $[82,83]$ or sustained by the sedative drugs which accumulate over time.

\section{The Effect of Sedative Therapies}

Cerebral blood flow is coupled to $\mathrm{CMRO}_{2}$ which, in turn, is coupled to neuronal activity [40]. Sedatives and anesthetics may reduce $\mathrm{CBF}$ by depressing neuronal activity and consequently $\mathrm{CMRO}_{2}$ and CMRG [87]. Infusion with barbiturate [42] or propofol $[88,89]$ and high doses of benzodiazepine can reduce CBF in a substantially coupled manner. Sedation is applied in most patients so that its impact on $\mathrm{CBF}$ cannot be isolated. However, once barbiturate and propofol are added to control refractory intracranial hypertension, a significant $\mathrm{CBF}$ depression can occur as shown in Fig. (24). Summary data confirm lower median CBF values in patients with continuous barbiturate infusion (Fig. 25). The reduction in $\mathrm{CBF}$ due to sedatives interferes with the interpretation of decreased CBF due, primarily, to low CPP [58] or to the trauma itself $[41,52,83]$. In fact, $\mathrm{CMRO}_{2}$ is naturally reduced after TBI [40, 41, 82]. The clear relationship between $\mathrm{CMRO}_{2}$ and GCS $[40,51,76]$ suggests that $\mathrm{CMRO}_{2}$ is naturally related to initial trauma severity. Data from our observations show, as expected, no association between best GCS before ICU admission and CBF (Fig. 26, results from $505 \mathrm{Xe}-\mathrm{CT}$ studies, unpublished data). However, from the same data only a slight association between reduced $\mathrm{CMRO}_{2}$ and the best GCS was found (Fig. 27, results from 393 Xe-CT studies, unpublished data), most likely owing to the practice to sedate patients with benzodiazepine and opioids [90,91], and the fact GCS is less reliable in the actual initial care context $[92,93]$. All these findings may even explain why it is so difficult to find clear CBF thresholds for the prediction of ischemia [4]. Hypothermia is a further depressor of $\mathrm{CBF}$ that should be borne in mind during CBF interpretation (Fig. 28) [94]. 


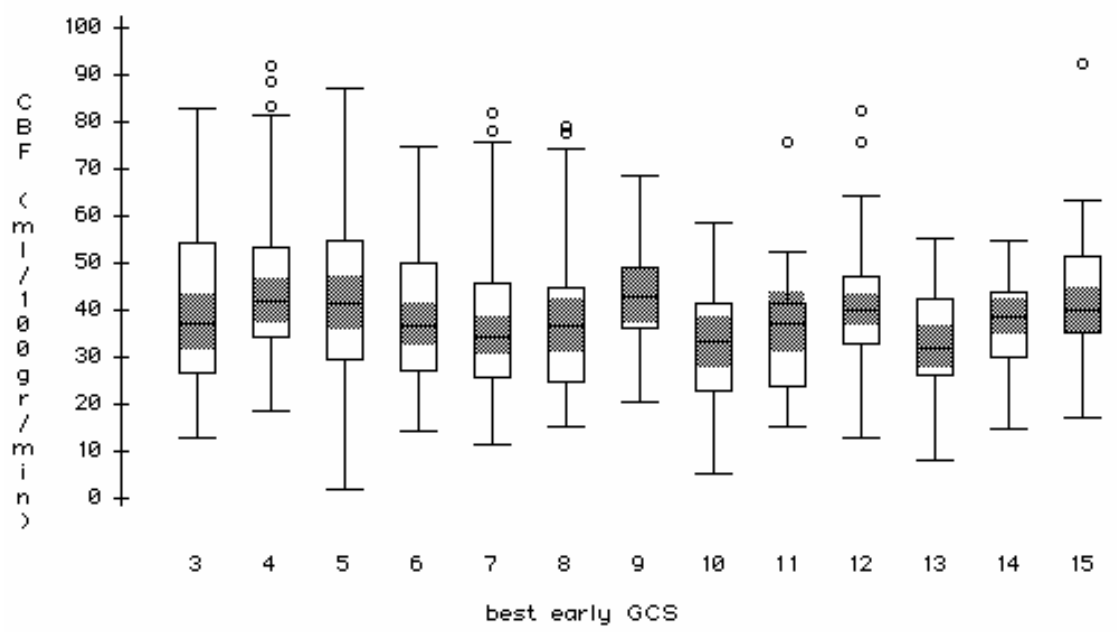

Fig. (26). No association has been found between the best Glasgow Coma Scale score (GCS) values observed the first day post injury and CBF values. Data from $505 \mathrm{Xe}-\mathrm{CT}$ studies (unpublished data). Grey rectangles represent the 95\% confidence interval for comparing median values.

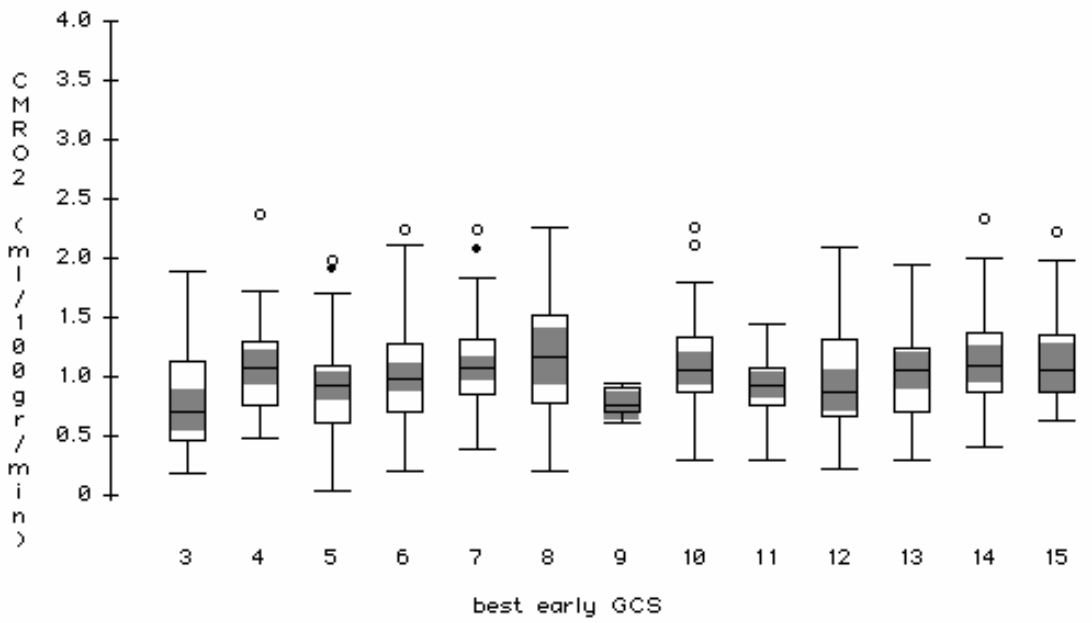

Fig. (27). A slight association has been found among best Glasgow Coma Scale score (GCS) values observed the first day post injuryand lower cerebral metabolic rate of oxygen $\left(\mathrm{CMRO}_{2}\right)$ values. Data from 393 Xe-CT studies (unpublished data). Grey rectangles represent the $95 \%$ confidence interval for comparing median values.

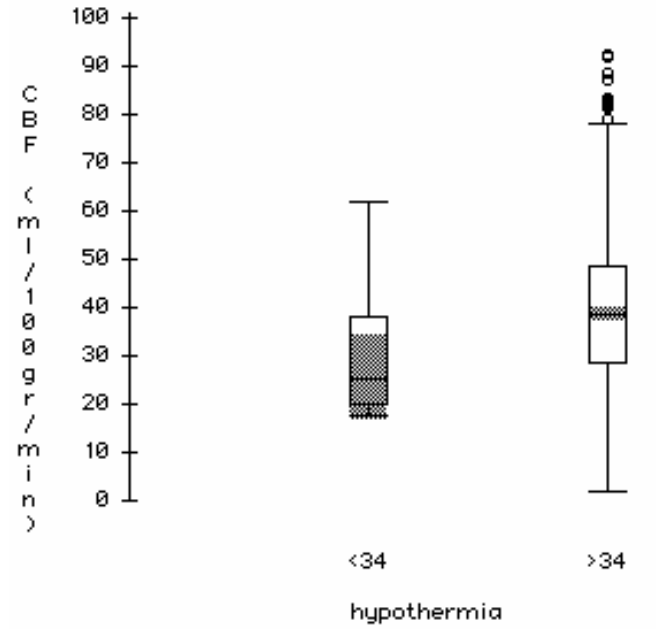

Fig. (28). Reduced median global CBF values Xe-CT studies during hypothermia. Data from $546 \mathrm{Xe}-\mathrm{CT}$ studies (unpublished data) obtained in patients managed with core temperature below $34{ }^{\circ} \mathrm{C}$. Grey rectangles represent the $95 \%$ confidence interval for comparing median values.

\section{Quantitative Interpretation of Global CBF}

Normal CBF values are a statistic concept more than a biological one. In TBI patients, a normal (Gaussian) distribution of CBF is expected and consequently "normal" values may be derived. Considering only patients between 15 and 65 years (448 Xe-CT studies, unpublished data), we found a mean CBF value of $40.0 \pm 15.7 \mathrm{ml} / 100 \mathrm{gr} / \mathrm{min}$ (Fig. 29). Such CBF values do not seem to be different from those reported by Glenn et al. $(40.2 \pm 13.2 \mathrm{ml} / 100 \mathrm{gr} / \mathrm{min})$ which were lower than those measured in normal volunteers in the same institution $(46.2 \pm 10.5 \mathrm{ml} / 100 \mathrm{gr} / \mathrm{min})$ using the intravenous ${ }^{133}$ Xenon clearance technique [82]. One way of interpreting $\mathrm{CBF}$ is to try to understand, by means of a multiple regression analysis, the several variables which affect $\mathrm{CBF}$ (Table 1). In a series of 423 studies, age, Xe-CT measurements within 24 hrs post injury, hematocrit, barbiturates and ICP negatively correlated with global CBF values which positively correlated with $\mathrm{PaCO}_{2}$ values. Surprisingly, CBF did not correlate with CPP (Fig. 30), as previously reported in a smaller series [95] and confirmed by others [55, 96, 97]. These findings may be accounted for in various ways. The 


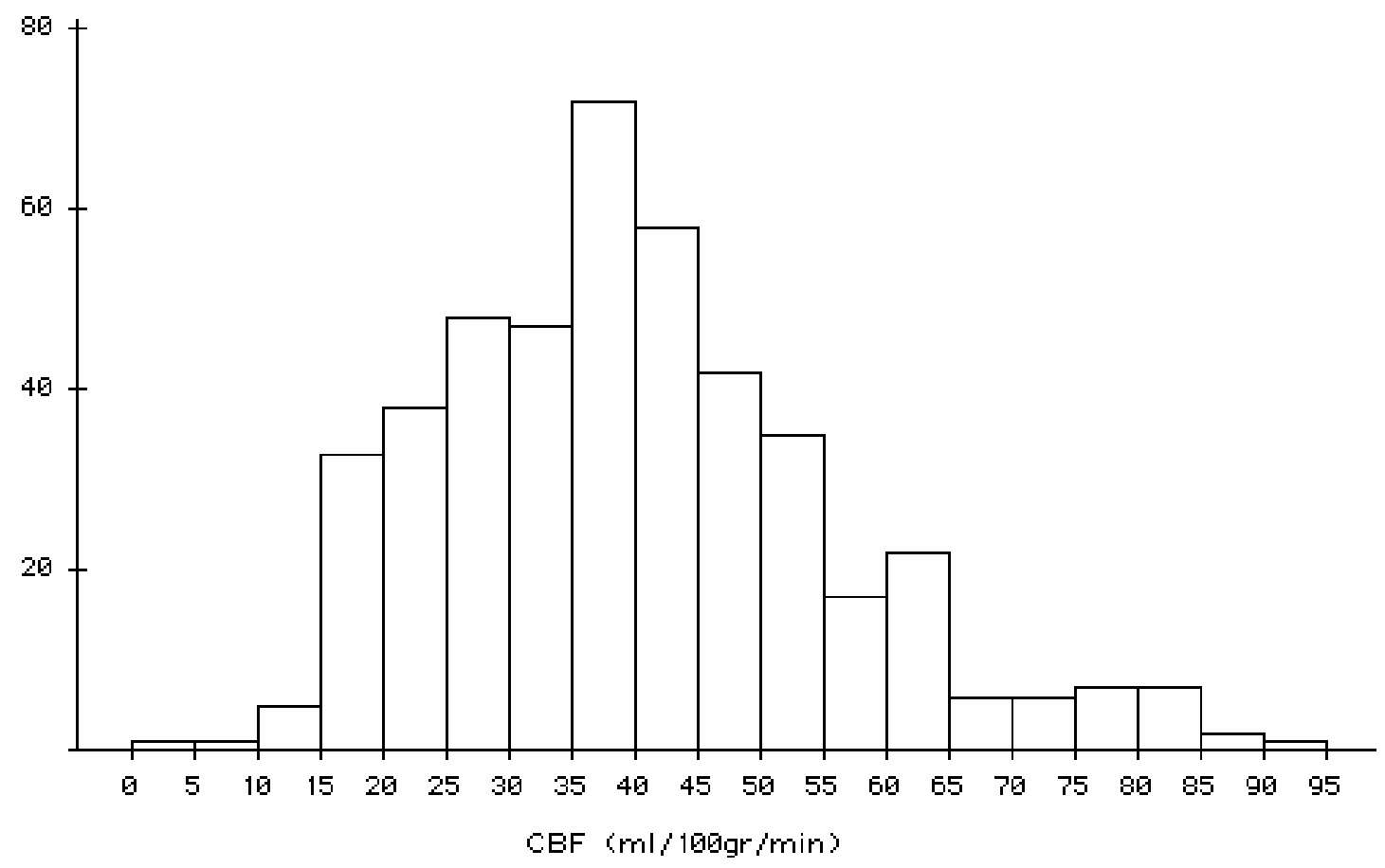

Fig. (29). Distribution of global CBF in adults (15-65 years, mean CBF value $40.0 \pm 15.7 \mathrm{ml} / 100 \mathrm{gr} / \mathrm{min}$ ). Data from $448 \mathrm{Xe}-\mathrm{CT}$ studies (unpublished data).

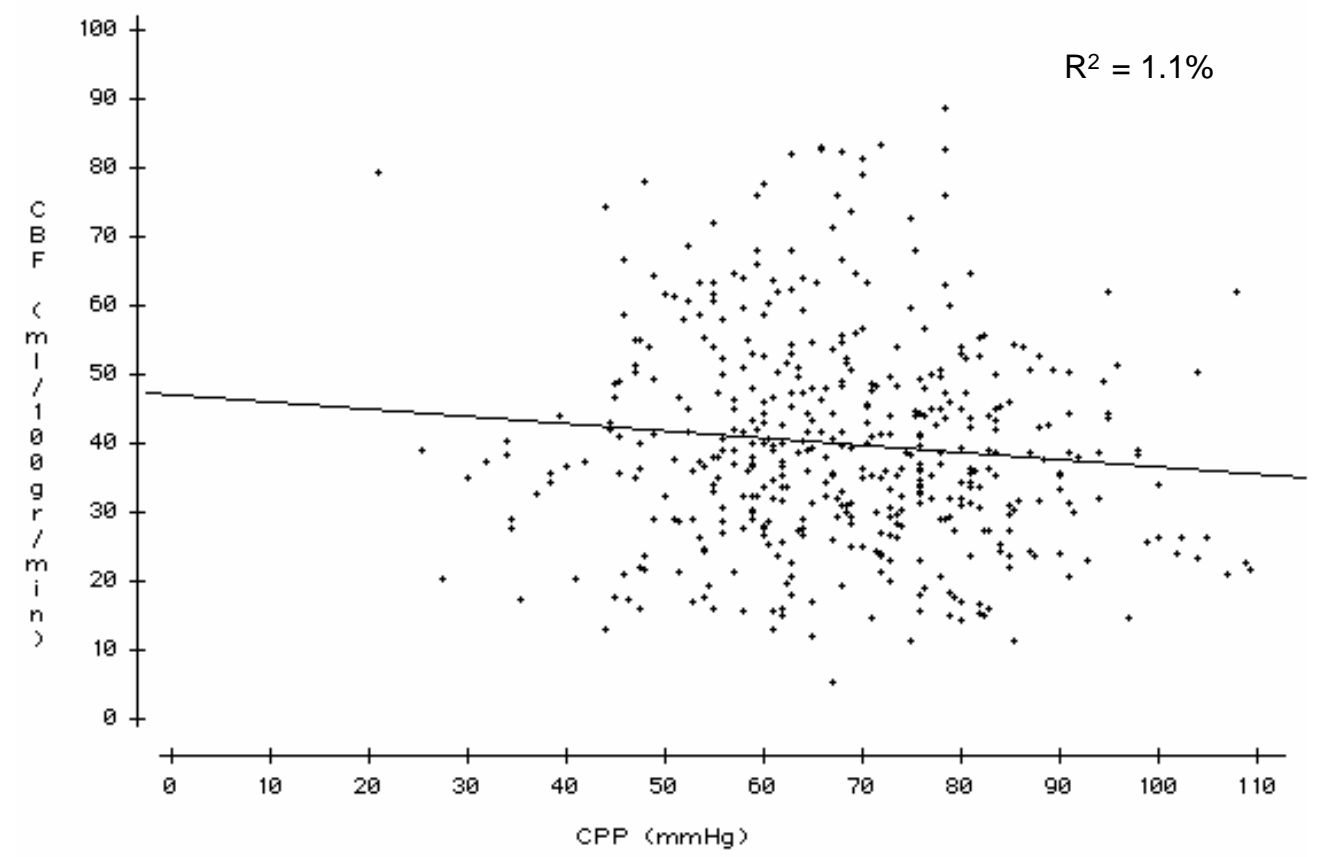

Fig. (30). Scatterplot between cerebral perfusion pressure (CPP) and global CBF. No correlation was found; the value of R Squared (1.1\%) was very low. Data from $423 \mathrm{Xe}-\mathrm{CT}$ studies (unpublished data).

main reason might be that most patients are physiologically located in the middle of the autoregulatory curve so that $\mathrm{CBF}$, once ICP and mean arterial pressure (MAP) have been adequately managed, depends more on trauma severity and $\mathrm{CMRO}_{2}$ rather than on systemic variables, at least in selected patients. Such results are affected by case mix selection and the time frame in which the CPP and CBF relationship is observed. In fact, as shown by Hlatky et al. [58], a remarkable relationship between $\mathrm{CPP}$ and $\mathrm{CBF}$ was shown in the early phases post injury, most likely as a result of the predominance of patients with low CPP values and deranged autoregulation.

The multiple regression shown in Table 2 can be improved in its prediction to $41 \%$ by adding $\mathrm{AVDO}_{2}$ to variables (377 Xe-CT studies, unpublished data). However, we can consider that $\mathrm{AVDO}_{2}$ values depend on $\mathrm{CBF}$ and $\mathrm{CMRO}_{2}$ levels, and not vice-versa [98]. In spite of this, $\mathrm{AVDO}_{2}$ remains of interest as an estimate of global CBF 
Table 1. Variables predictive of the global CBF in $423 \mathrm{Xe}-\mathrm{CT}$ studies in severe and moderate TBI patients ventilated and sedated (unpublished data). This model explains $30.4 \%$ of the global CBF values.

\begin{tabular}{|c|c|c|}
\hline \multicolumn{2}{|c|}{ R squared (adjusted) $\mathbf{3 0 . 4 \%}$} \\
\hline \hline Variable & Coefficient & prob \\
\hline Constant & 53.4909 & $\leq 0.0001$ \\
\hline Age (years) & -0.247933 & $\leq 0.0001$ \\
\hline Measurement within 24 hr post injury & -4.40421 & $\leq .0479$ \\
\hline Htc (\%) & -1.1577 & $\leq 0.0001$ \\
\hline PaCO $($ mmHg) & 0.83723 & $\leq 0.0001$ \\
\hline ICP mean (mmHg) & -0.101575 & 0.0881 \\
\hline Continuous barbiturate infusion & -6.97103 & 0.0414 \\
\hline
\end{tabular}

ICP mean: the mean hourly intracranial pressure (ICP) measured the same day of Xe-CT study.

barbiturate: continuous infusion of barbiturate in association with benzodiazepine (day of the study).

Table 2. Variables predictive, including Arterial-Venous $\mathrm{O}_{2}$ content difference $\left(\mathrm{AVDO}_{2}\right)$, of the global CBF in $377 \mathrm{Xe}-\mathrm{CT}$ studies in severe and moderate TBI patients ventilated and sedated (unpublished data). This model explains $41.2 \%$ of the global CBF values.

\begin{tabular}{|c|c|c|}
\hline \multicolumn{2}{|c|}{ R Squared (Adjusted) $\mathbf{4 1 . 2 \%}$} & prob \\
\hline \hline Variable & Coefficient & $\leq 0.0001$ \\
\hline Constant & 72.9446 & $\leq 0.0001$ \\
\hline Age (years) & -0.179419 & $\leq 0.0001$ \\
\hline Ht (\%) & -0.597251 & 0.0435 \\
\hline ICP mean (mmHg) & -0.113539 & 0.0030 \\
\hline Continuous barbiturate infusion & -9.66229 & 0.0377 \\
\hline Continuous propofol infusion & -2.66441 & 0.0729 \\
\hline PaCO $_{2}(\mathrm{mmHg})$ & 0.229551 & $\leq 0.0001$ \\
\hline $\mathrm{AVDO}_{2}(\mathrm{ml} / \mathrm{dl})$ & -4.88004 & \\
\hline
\end{tabular}

ICP mean: the mean hourly intracranial pressure (ICP) measured the same day of Xe-CT study.

Continuous barbiturate infusion: continuous infusion of barbiturate in association with benzodiazepine and fentanyl (day of the study).

Continuous propofol infusion: continuous infusion of propofol in association with benzodiazepine and fentanyl (day of the study).

(Fig. 31), with a correlation factor similar to that obtained with a combination of multiple variables such as age, measurements within 24 hrs post injury, hct, barbiturate, ICP and $\mathrm{PaCO}_{2}$ values (Table 1). It seems, therefore that basic clinical information can only partially explain and predict global $\mathrm{CBF}$ values, suggesting that additional variables should be investigated, although our potential to understand global $\mathrm{CBF}$ is still limited. Further, while only barbiturates seem to affect $\mathrm{CBF}$ in multivariate analysis, it is unclear, however, whether increasing the dosage would result in further CBF reduction $[95,99]$. In fact, such a hypothesis is not supported by our data showing that $\mathrm{CBF}$ is not significantly lower in more intensely treated patients, which may be a consequence of uncoupling from oxygen metabolism (Fig. 32). Conversely, $\mathrm{CMRO}_{2}$ is reduced in Xe-CT studies performed in patients in extreme therapy for refractory intracranial hyper- tension (Fig. 33). However, in the interpretation of data it has to be remembered that deeper sedation delivered over a longer period is commonly used in more severe patients with higher ICP levels. Since severity is intrinsically associated with lower $\mathrm{CMRO}_{2}$ values [40], lower $\mathrm{CMRO}_{2}$ in these patients could be fundamentally cross linked to a treatment associated $\mathrm{CMRO}_{2}$ depression.

Physiological variables may contribute to the definition of trauma outcome. It is well established that low global CBF values have some relation to early death [38,59] and with an unfavorable outcome $[59,76]$ only in the first hours post injury. In our population (Fig. 34), patients who died had reduced $\mathrm{CBF}$ values. One of the reasons explaining why, overall, $\mathrm{CBF}$ is a poor predictor of outcome is that after the first 24 hours uncoupling predominates, as shown by the increase in $\mathrm{AVDO}_{2}$ [80]. The reasons why CBF is uncoupled 


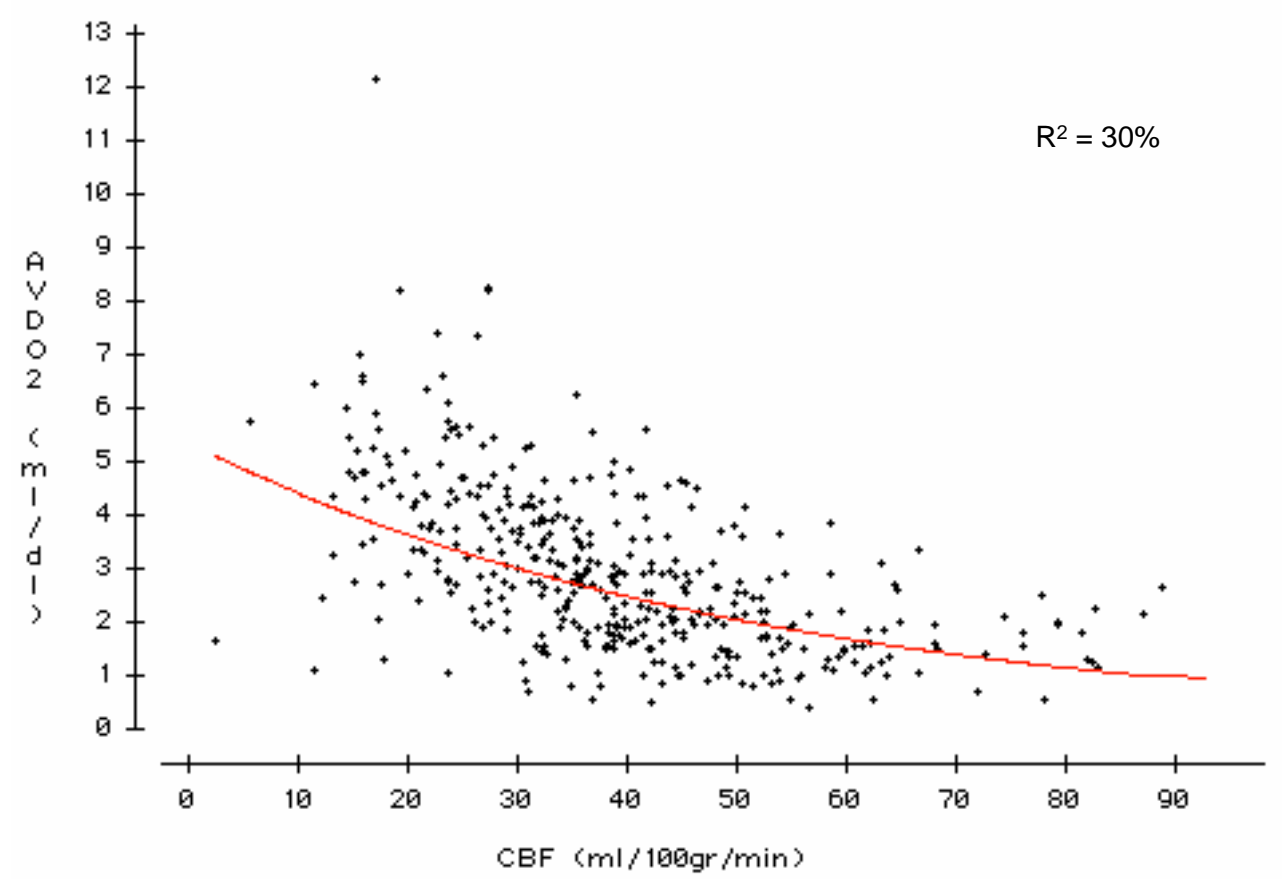

Fig. (31). Scatterplot between global CBF and arterio-venous oxygen contents difference (AVDO2). Data from 395 Xe-CT studies (unpublished data).

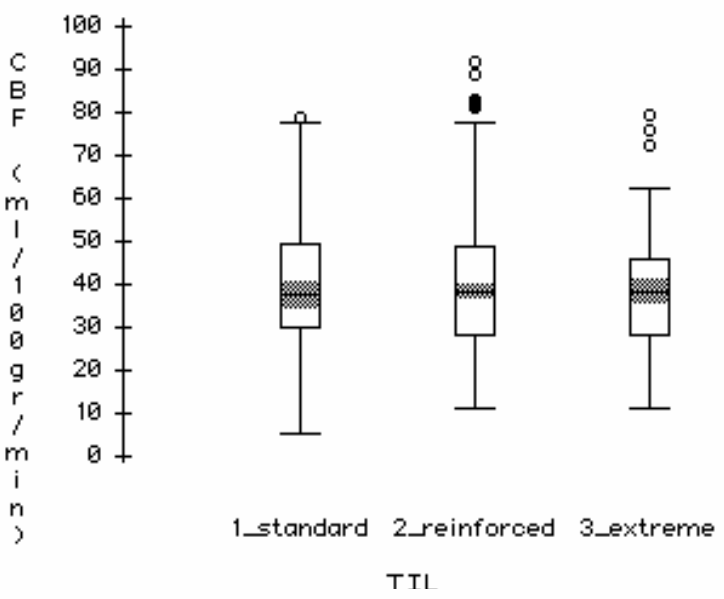

Fig. (32). CBF values do not seem to be associated to the Therapeutic Intensity Level (TIL). Data from 447 Xe-CT studies (unpublished data). Grey rectangles represent the $95 \%$ confidence interval for comparing median values.

from metabolism are complex and might involve disturbance in autoregulation, early ischemia with elevated oxygen extraction, irreversible ischemia with defective oxygen extraction, hypometabolism or coupling with glucose rather than oxygen. While CBF is poorly informative regarding outcome, through global CBF measurement it is possible to calculate global $\mathrm{CMRO}_{2}$, which has been found to be reduced in TBI in several studies [40]. Patients with better Glasgow Outcome Scale [100] at one year are associated with wider $\mathrm{AVDO}_{2}$ (Fig. 35) and higher $\mathrm{CMRO}_{2}$ values (Fig. 36). These results seem to complete the observation coming from Stocchetti et al., who demonstrated that wider $\mathrm{AVDO}_{2}$ values are associated with improved outcomes [101].
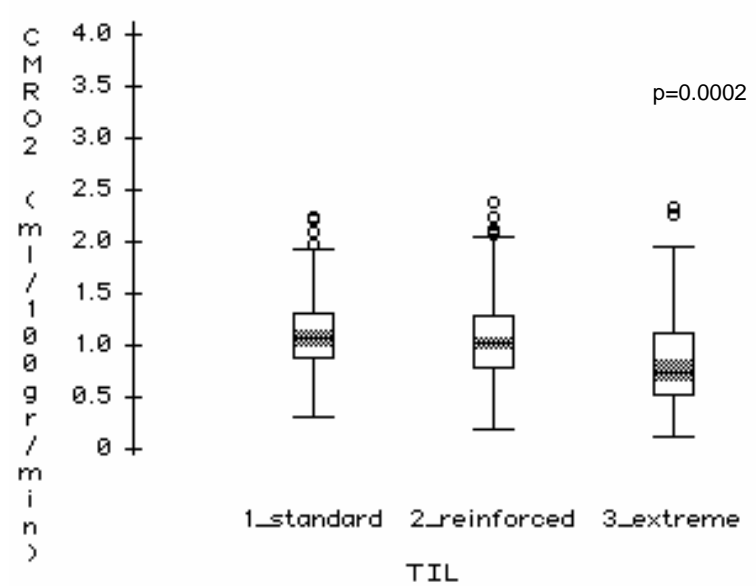

Fig. (33). Cerebral metabolic rate of oxygen $\left(\mathrm{CMRO}_{2}\right)$ values seem to be associated to the Therapeutic Intensity Level (TIL). A lower $\mathrm{CMRO} 2$ value was found in Xe-CT studies obtained with the highest (extreme) therapeutic level. Data from $373 \mathrm{Xe}-\mathrm{CT}$ studies (unpublished data). Grey rectangles represent the $95 \%$ confidence interval for comparing median values.

\section{Bedside Interpretation of Global CBF Values}

Bearing in mind all the aforementioned considerations, we can now attempt to describe an oversimplified approach based on low and high global CBF values. Even if the visual perception may be different from the quantitative one, for instance, we can consider low $\mathrm{CBF}$ values as those below 18 $\mathrm{ml} / 100 \mathrm{gr} / \mathrm{min}$ and high $\mathrm{CBF}$ values as those above 33 $\mathrm{ml} / 100 \mathrm{gr} / \mathrm{min}$ [49-51].

Global low CBF is one of the most difficult findings to interpret. In fact, ageing, the severity of trauma and deep sedation with metabolic suppressive drugs may affect $\mathrm{CBF}$ 


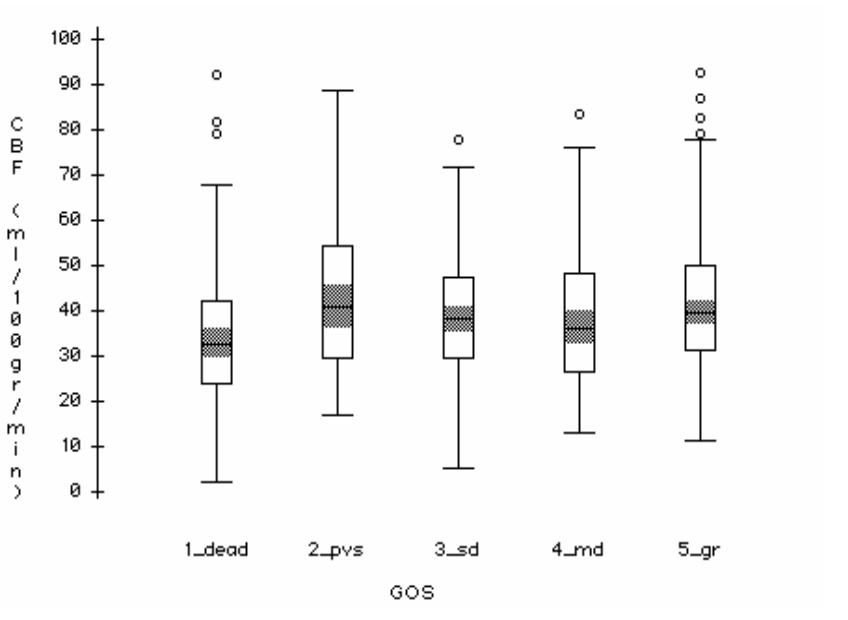

Fig. (34). Lower CBF values can be observed in patients who eventually died. Overall, a poor association can be observed between CBF and outcome expressed by means of GOS [100] at 12 months. Data from $479 \mathrm{Xe}-\mathrm{CT}$ studies (unpublished data). Grey rectangles represent the $95 \%$ confidence interval for comparing median values.

Legend: pvs: persistent vegetative state; sd: severe disability; md: moderate disability; gr: good recovery.

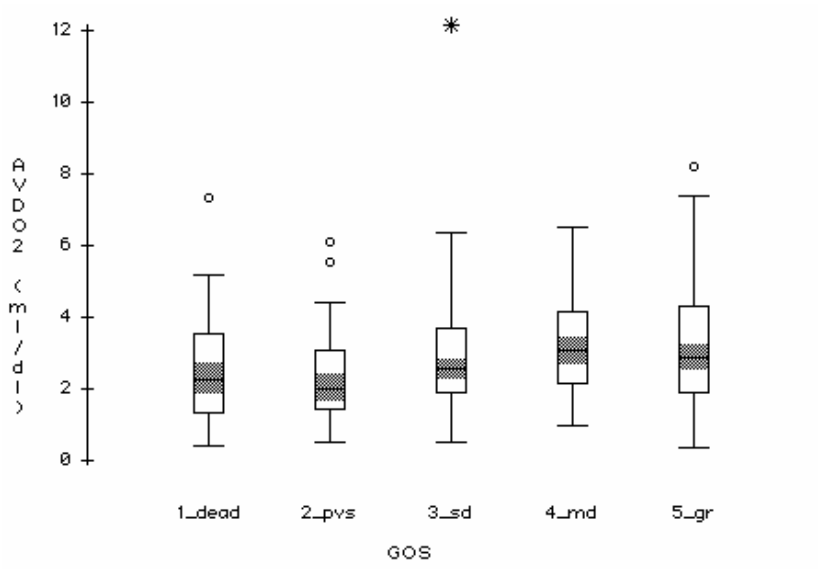

Fig. (35). A progressive increase in arterial-venous oxygen contents difference $\left(\mathrm{AVDO}_{2}\right)$ can been observed in association with better Glasgow Outcome Scale (GOS) categories [100]. Data from 373 Xe-CT studies (unpublished data). Grey rectangles represent the 95\% confidence interval for comparing median values.

Legend: pvs, persistent vegetative state; sd: severe disability; md: moderate disability; gr: good recovery.

with a coupled reduction, without ischemic decline in oxygen consumption. In Fig. (37) are reported four cases with CBF decline, but only one patient evolved to brain death due to global hypoperfusion.

Low global CBF levels are more frequently found in the early hours post injury $[38,59]$. Some of these cases are associated to true ischemia as shown by the strong association with early mortality [38,59] and low CPP values [58]. Global low $\mathrm{CBF}$ values can be found in patients with not evacuated $\mathrm{SDH}$ [102]. This finding is probably common in comatose

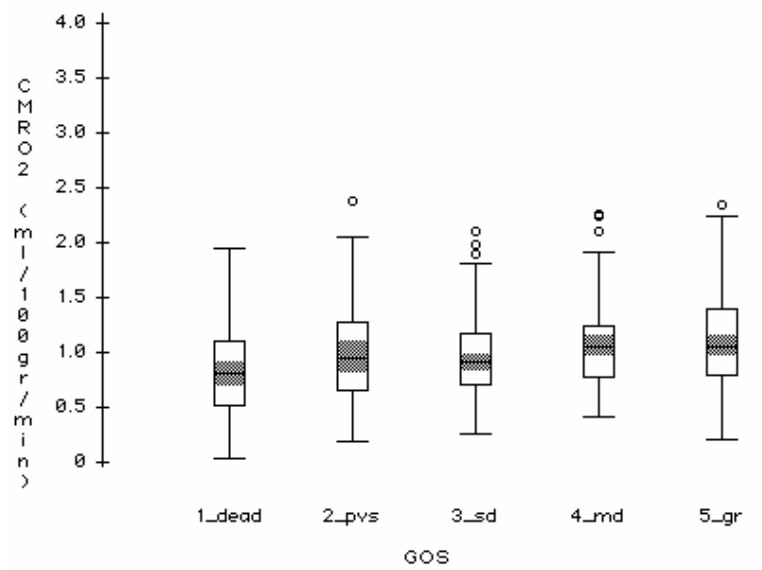

Fig. (36). A progressive increase of cerebral metabolic rate of oxygen (CMRO2) can been observed in association with improved outcome categories expressed by means of GOS at 12 months [100] (findings from $373 \mathrm{Xe-CT}$ studies, unpublished data). Grey rectangles represent the $95 \%$ confidence interval for comparing median values.

Legend: pvs: persistent vegetative state; sd: severe disability; md: moderate disability; gr: good recovery.

patient with not evacuated mass lesion, but they are rarely described in the literature because measurement of CBF before SDH evacuation should not introduce delays in prompt mass evacuation.

High global CBF: this is a not frequent condition. It is more frequent in younger people, in whom it is physiologically justified. Global high CBF values seem to be associated with diffuse brain injury (Fig. 38) and, less frequently, with post-anoxic encephalopathy [103]. According to Kelly [45] high global $\mathrm{CBF}$ values are more associated with high ICP and poor outcome.

\section{Interpretation of Regional CBF}

In TBI stabilized lesions are commonly analysed: posttraumatic cerebral infarction, the contusion/laceration, the subdural hematomas, the intraparenchymal hematoma.

\section{Posttraumatic Cerebral Infarction (PTCI)}

PTCI has recently been described in a paper by the Latronico group [103] and includes: $1^{\text {st }}$ territorial cerebral infarction, complete or incomplete; $2^{\text {nd }}$ watershed cerebral infarction; $3^{\text {rd }}$ non-territorial non-watershed cerebral infarction. The most common example of territorial cerebral infarction is the hypodense lesion in the posterior cerebral artery, after an episode of coning. In such cases the rCBF may be low, as expected (Fig. 39) since compression of the afferent artery against the tentorium margin is the cause of macrovascular ischemia. However, high $\mathrm{rCBF}$ values instead of low $\mathrm{rCBF}$ values can sometimes be found (Fig. 40). We can pose the hypothesis that a short lasting compression of main trunk artery leads to a critical hypoperfusion promoting cytotoxic edema, expressed at CT by a hypodense area, but after the relief of the compression, a reperfusion occurs. Conversely, long lasting compression could be associated with no reflow. Finally, in some cases, the hypoattenuation may be associated with normally depressed rCBF (Fig. 41). Similar find- 


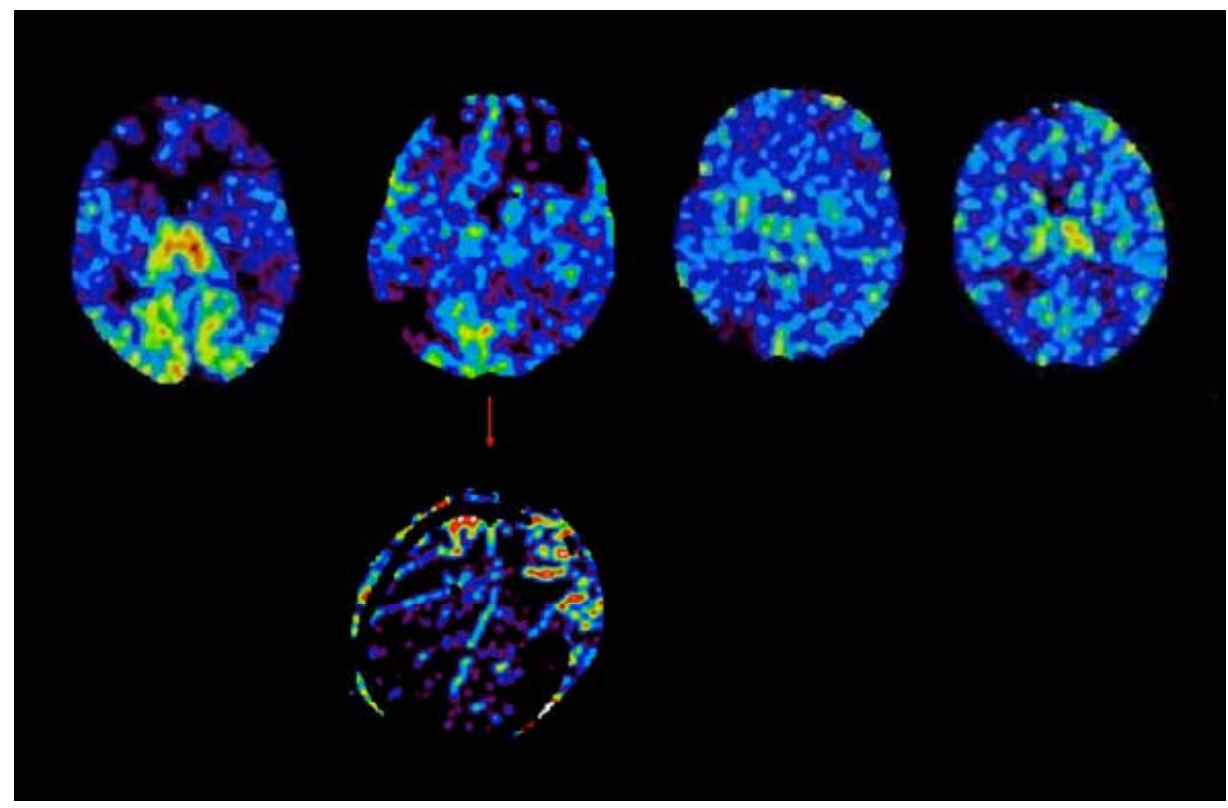

Fig. (37). Four cases with a low global CBF diffuse state. Only one patient evolved toward brain death. These series give an example of the difficulties in evaluating cases with diffusely reduced $\mathrm{CBF}$.

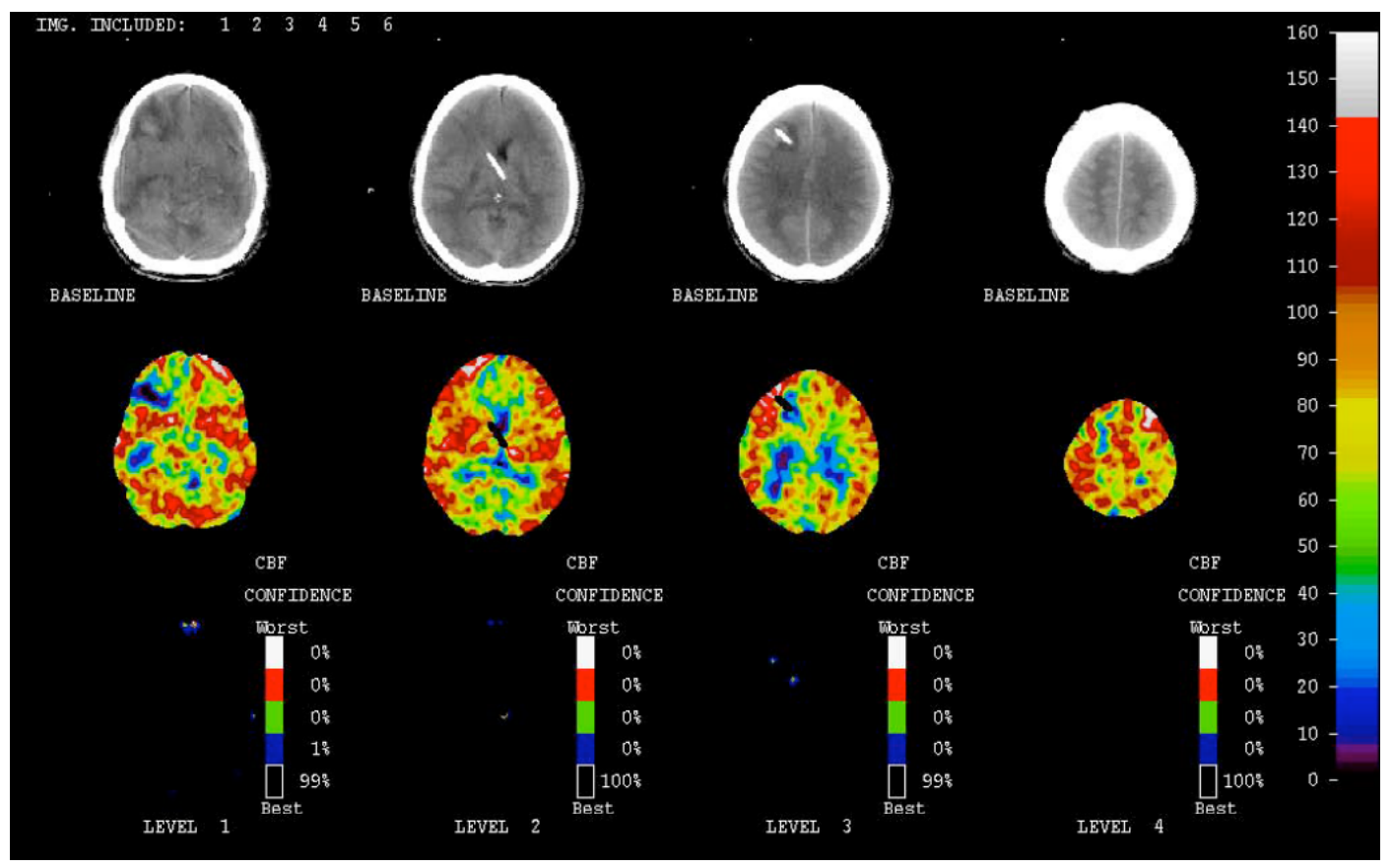

Fig. (38). Global hyperemia in patients with diffuse brain injury and diffuse brain swelling.

ings have been described by the Robertson group [104]. Watershed cerebral infarction can sometimes be found in patients with low CPP during the core phase in ICU management (Fig. 42). Non territorial non watershed infarction with multiple scattered hypoattenuation areas are sometimes found in patients who developed prolonged low CPP states [105]. These are more frequently due to primarily high ICP occurring in the early acute phase, sometimes before the evacuation of acute SDH [106], and presenting with severe clinical findings, GCS 3, bilateral midriasis, or in patients with initial secondary damage, hypoxia, hypotension, anemia. In patients affected by chronic arterial hypertension and intracranial hypertension, such areas may be the result of inappropriate CPP values in relation to a chronic rightward shift of autoregulation curve (Fig. 43).

\section{Traumatic Contusion/Laceration and Traumatic Hema- toma}

$\mathrm{CBF}$ in traumatic contusion and traumatic hematoma seems to be distributed in a concentric gradient consistent with an increase in $\mathrm{CBF}$ from the center to the periphery of the lesion. Schematically, the lesion may be subdivided into three ROIs: the hemorrhagic core, the perilesional low density area and the normal appearing area surrounding the edema. This method has been extensively described (Fig. 44) [75] and successfully applied with perfusion computerized tomography [107]. Among the various imaging modalities, 


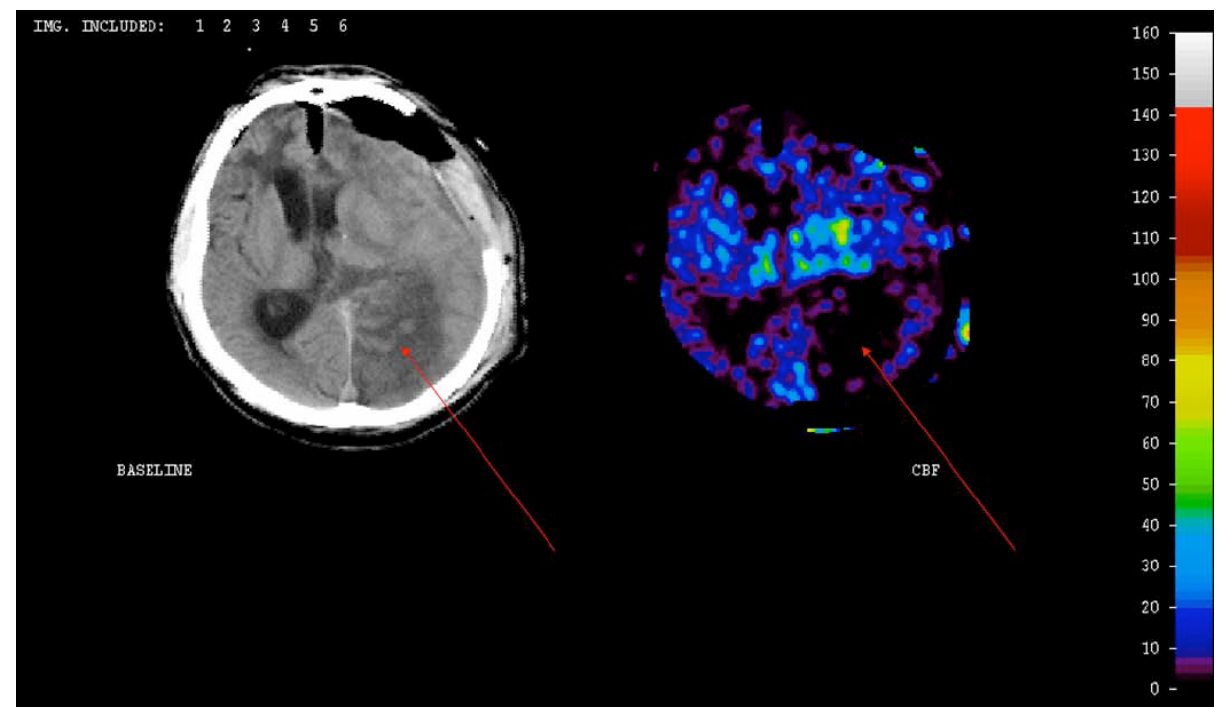

Fig. (39). Post traumatic cerebral infarction (PTCI) associated with deep ischemia. This is a territorial PTCI involving the posterior cerebral artery (PCA) supply territory.

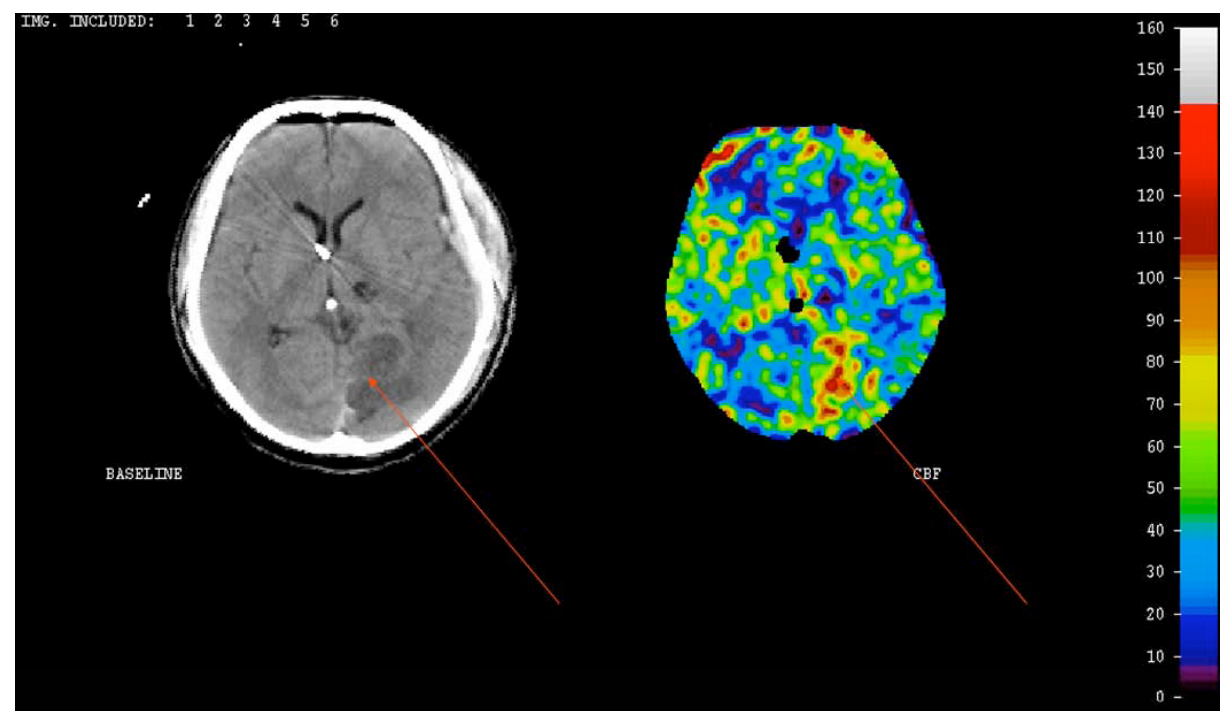

Fig. (40). Post traumatic cerebral infarction (PTCI) associated with focal hyperemia. This is a territorial PTCI involving the posterior cerebral artery (PCA) supply territory.

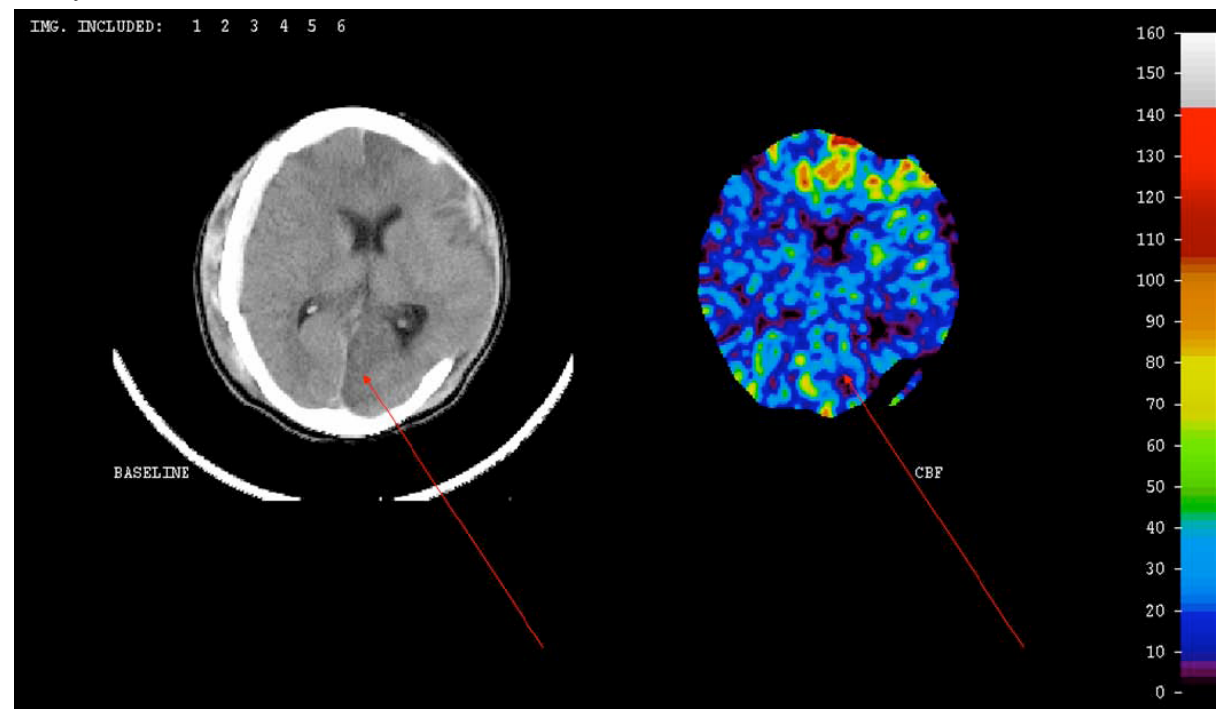

Fig. (41). Post traumatic cerebral infarction (PTCI) associated with mildly reduced CBF. This is a territorial PTCI involving the posterior cerebral artery (PCA) supply territory. 

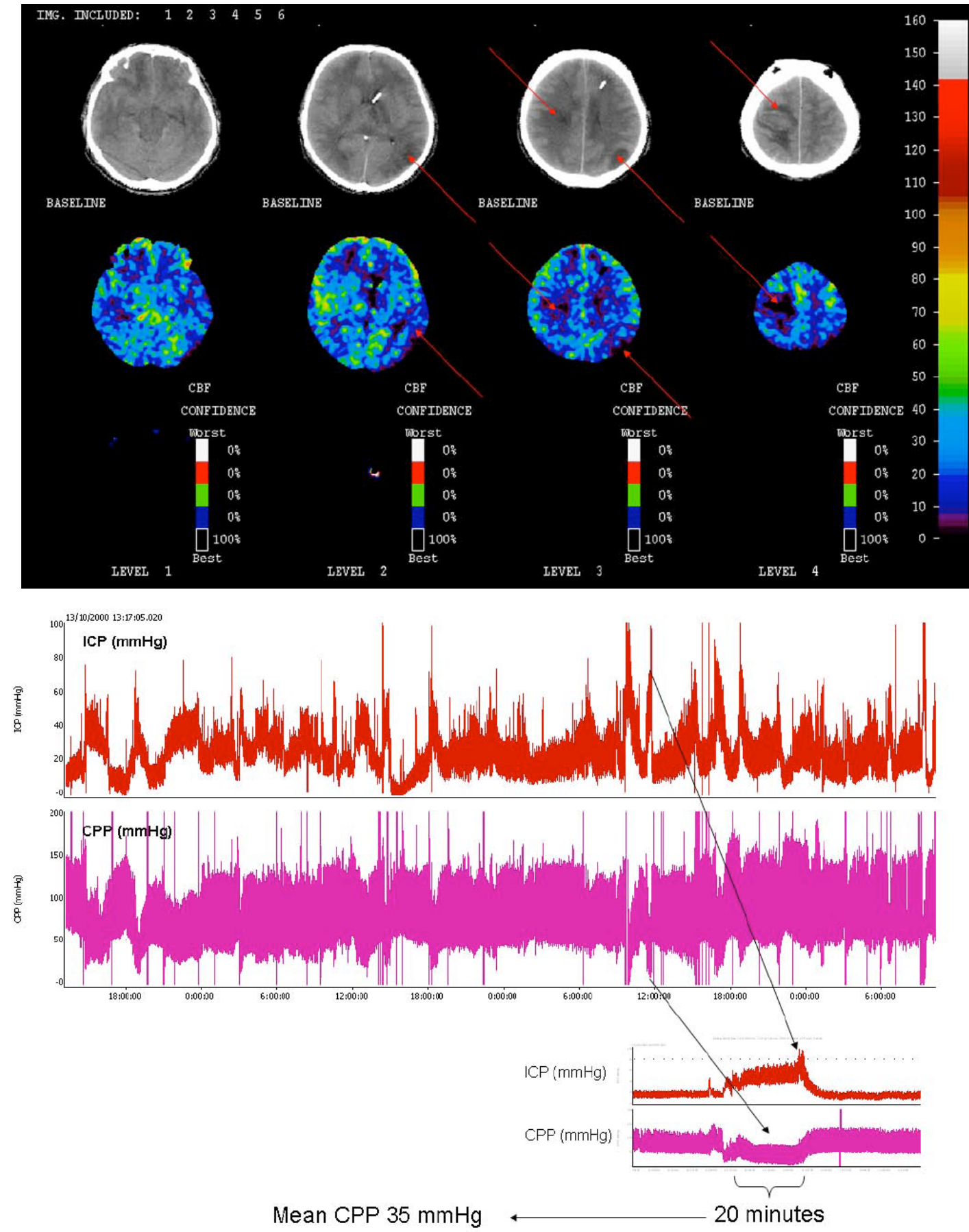

Fig. (42). Watershed post traumatic cerebral infarction (PTCI) involving the areas between posterior cerebral artery (PCA) and middle cerebral artery (MCA) and border zones within the white matter (arrows). Multiple infarctions were probably due to several drops in cerebral perfusion pressure (CPP), due to refractory increases in intracranial pressure (ICP).

$\mathrm{Xe}-\mathrm{CT}$ is the most employed technique for assessment of intraparenchymal lesions. Low rCBF values in the core and in the perilesional low density area are commonly found [36,53,104,108-110]. Intraparenchymal lesions are not a homogenous category and at least two macro subtypes can be distinguished [111]: traumatic contusions, in which the central edematous area surrounds or includes several bleeding focii, and traumatic hematomas, in which a well defined and prominent hemorrhagic core is circularly surrounded by edema. In 109 intraparenchymal lesions, we showed that low $\mathrm{CBF}$ values are more commonly found in the edematous area surrounding the hemorrhagic isles in traumatic contusion $(29.7 \pm 19.6 \mathrm{ml} / 100 \mathrm{gr} / \mathrm{min})$ than within the intralesional edematous area in traumatic hematoma (35.1 \pm 21.3 $\mathrm{ml} / 100 \mathrm{gr} / \mathrm{min}$ ) [112] Fig (45). These physiological findings suggest that the two lesions could be characterized by a different pathophysiology $[113,114]$. Presumably, the edematous-hemorrhagic core of the lesion is deeply affected by the primary destructive damage which includes a focal cerebral ischemia. Conversely, in traumatic intracranial hematomas, perilesional edema probably evolves towards an inflammatory reaction, with less $\mathrm{CBF}$ disturbance. 

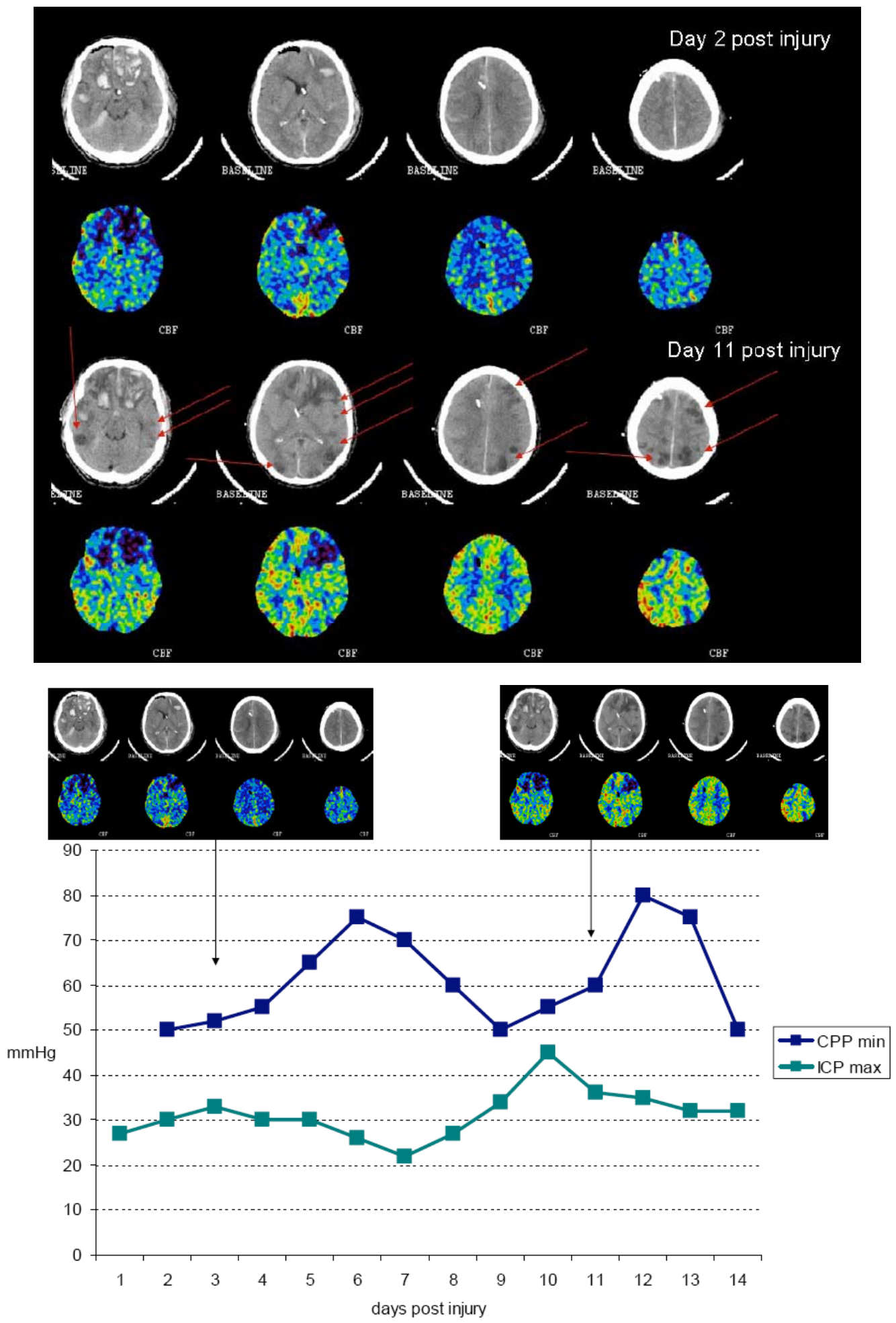

Fig. (43). A patient affected by chronic hypertension with bifrontal traumatic contusion developed intracranial hypertension. Mean daily values of cerebral perfusion pressure (CPP) were apparently adequate. However, the time course of the minimum daily CPP and the maximum intracranial pressure (ICP) values revealed values which were probably critical for patient physiology. The patient developed multiple scattered PTCI.

Decreased perfusion in intraparenchymal lesions represent a multifactorial phenomenon promoted by an increase in segmental arteriolar [115] and capillary resistance due to the swelling of the astrocytes endfoot [108], impairment of capillary network [116], and of oxygen diffusion [117]. Although correction of CBF impairment within and around the lesion seems to be indicated, the optimal therapeutic option remains to be defined. In this regard, Xe-CT may be able to verify the effect of physiological changes on intralesional rCBF. Hypocapnia, through hyperventilation, has been extensively applied to reduce intracranial hypertension. In fact, the vasoconstriction of pial arterioles and precapillary arteri- 


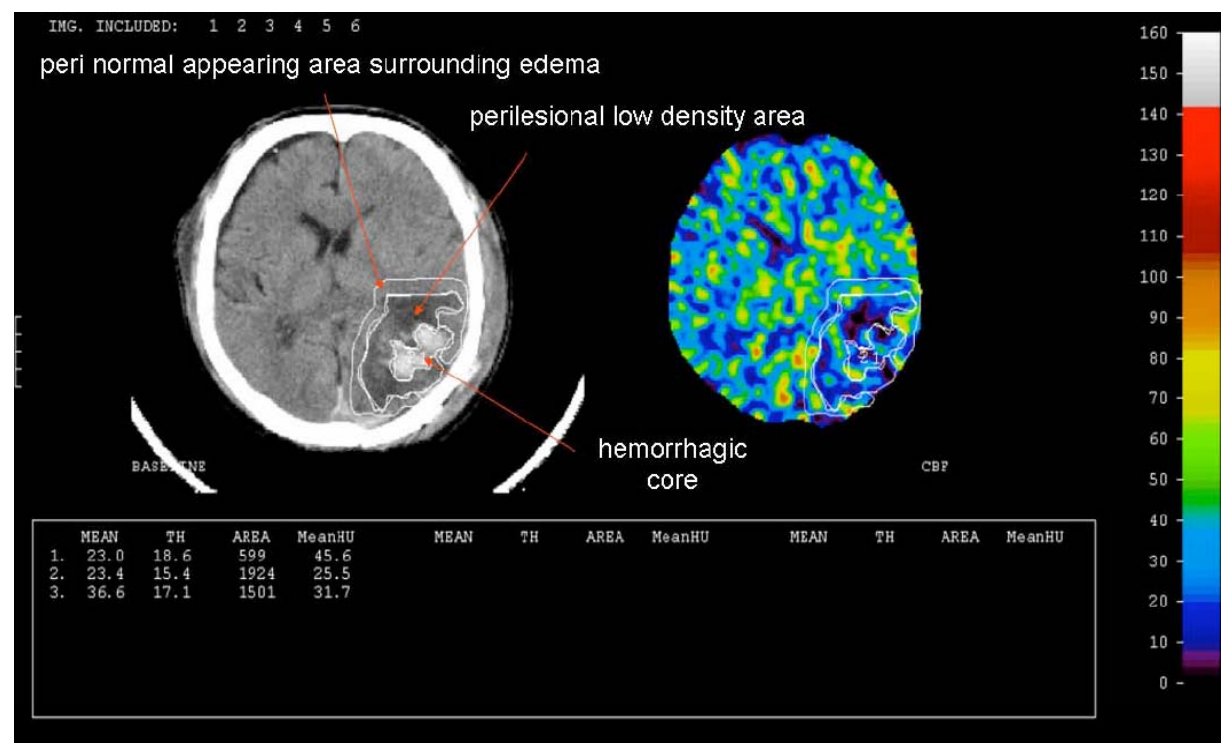

Fig. (44). Traumatic contusion and the ROI analysis according to Chieregato et al. [75]. Three different regions of interest (ROIs) larger than $1 \mathrm{~cm}^{2}$ were drawn freehand on the diagnostic CT: 1) within the traumatic hematoma proper (hemorrhagic core); 2) surrounding the hematoma low-density area (perihematoma low-density area); 3) within $1 \mathrm{~cm}$ of the normal-appearing brain tissue surrounding the perihematoma lowdensity area (perihematoma normal-appearing area).

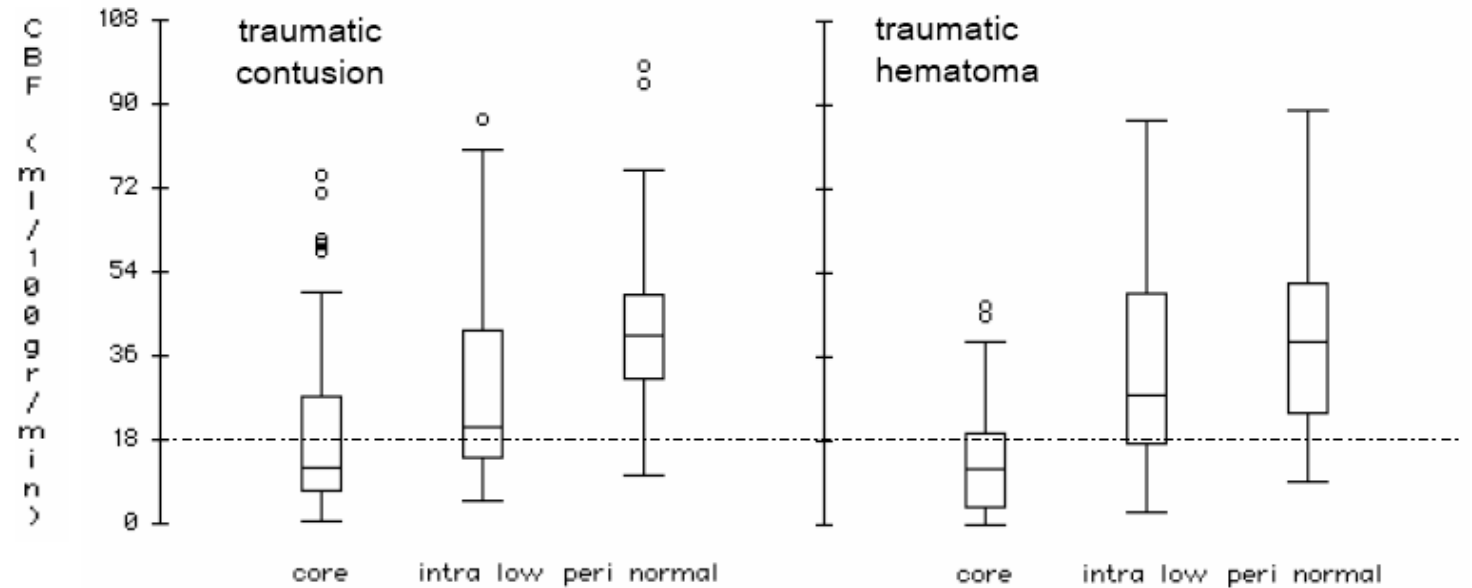

Fig. (45). Regional CBF values (on the y axis, CBF expressed in $\mathrm{ml} / 100 \mathrm{gr} / \mathrm{min}$ ) from intraparenchymal lesion measured in hemorrhagic core (core), in perihematoma low-density area (intra low), in perihematoma normal-appearing area (peri normal).

It may be observed that median values of rCBF in intralesional edema (intra low) are lower in traumatic contusion toward intraparenchymal traumatic hematoma. Data from $125 \mathrm{Xe}-\mathrm{CT}$ studies.

The dashed line indicate the traditional CBF threshold of ischemia of $18 \mathrm{ml} / 100 \mathrm{gr} / \mathrm{min}$ [49].

oles reduces the cerebral blood volume and, as a function of the cerebral compliance, reduces ICP as well [118]. However, hypocapnia can also result in CBF reduction. Marion [53] and McLaughlin [52], by means of double test change of $\mathrm{PaCO}_{2}$, showed that, beside global reduction in $\mathrm{CBF}$, hypocapnia is also responsible for $\mathrm{rCBF}$ decrease in the pericontusional edematous area. While doubts have been reported on the real consistence of ischemia related to hypocapnia in apparently normal tissue [119], edematous areas seem sensitive to increases in $\mathrm{PaCO}_{2}$ which may be associated with small improvements in intralesional rCBF (Fig. 46).

A further therapy, commonly used by neurointensivists, is the induction of an increase in CPP to control ICP [120,121], and to improve local rCBF [122]. Regarding focal hypoperfusion, however, clinical studies by means of PET have shown only minor improvements in $\mathrm{rCBF}$ in intralesional edematous area following CPP elevation [123]. In a subsequent study [57], we showed that only intraparenchymal lesions with low critical rCBF within the intralesional edematous area can gain a minor improvement (Fig. 47). This increase, however, was too shallow to be of clinical relevance. Conversely, patients with relatively high rCBF showed a consistent reduction in $\mathrm{rCBF}$ in response to $\mathrm{CPP}$ elevation. It may, therefore, be hypothesized that microcirculation becomes structurally affected by ischemia with associated impaired autoregulation in patients with low rCBF. However, due the paucity of recruitable microvessels, only a minor improvement can be obtained. Conversely, when a preserved baseline $\mathrm{rCBF}$ is found in areas with vasogenic edema [124] and a damaged blood brain barrier (BBB), a sudden increase in CPP could be responsible for a further BBB breakdown [125] and noradrenaline diffusion outside 


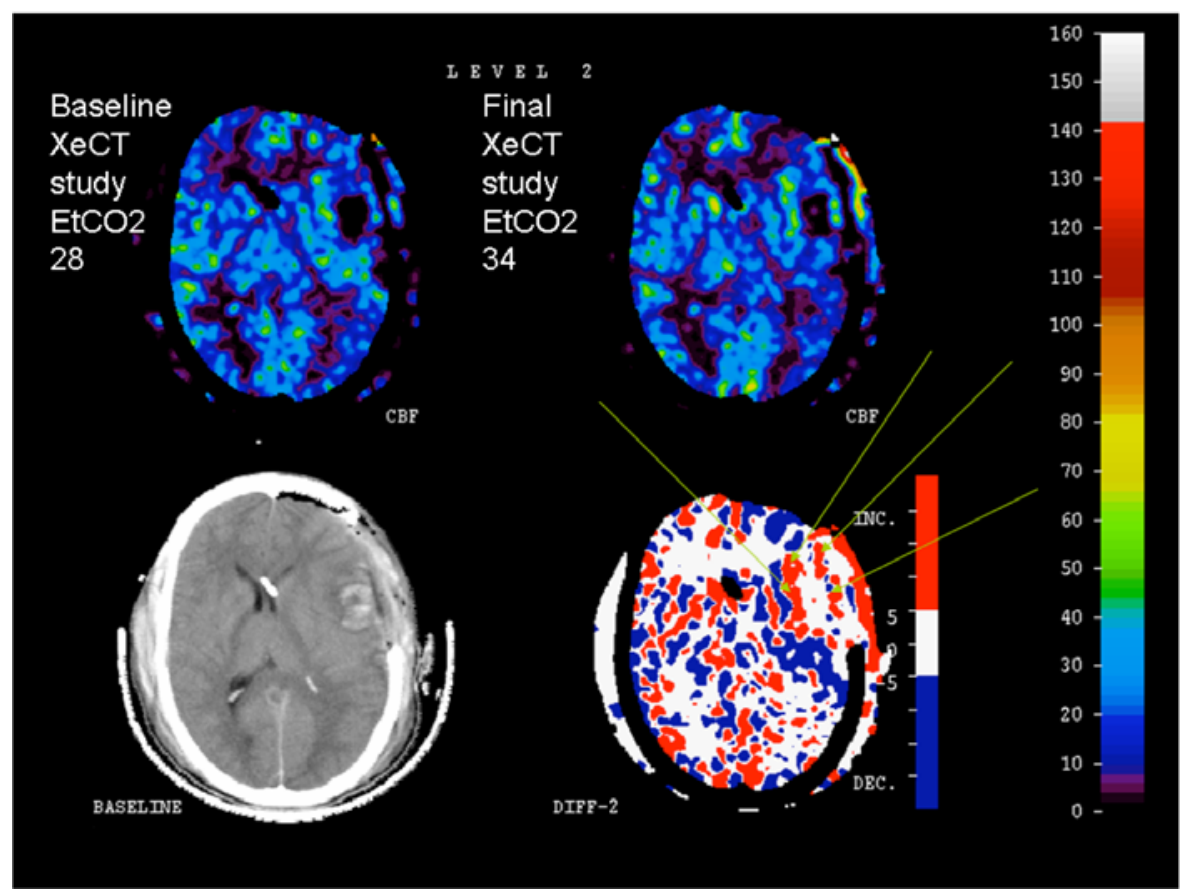

Fig. (46). A patient with a satellite contusion below an evacuated subdural hematoma (SDH). An induced increase in end tidal $\mathrm{CO}_{2}\left(\mathrm{EtCO}_{2}\right)$ was induced (from 28 to $34 \mathrm{mmHg}$ ) and was associated with an improvement in rCBF in the traumatic contusion (arrows).
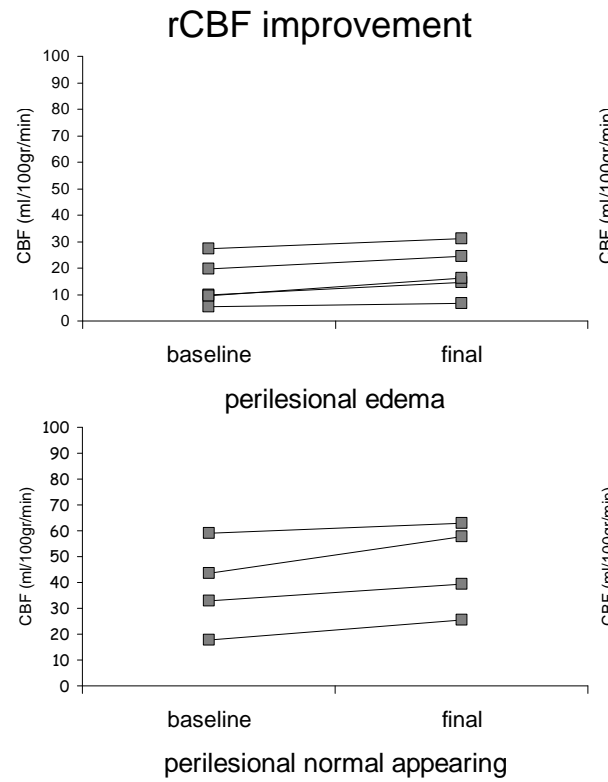

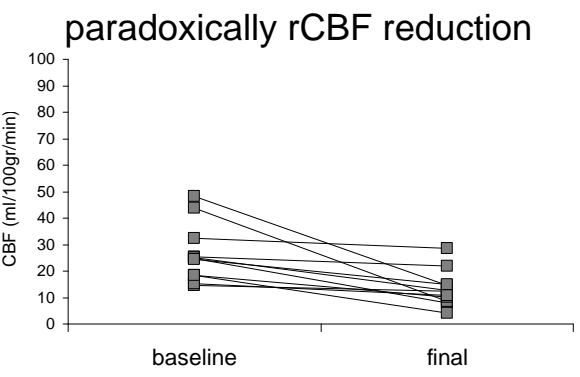

perilesional edema

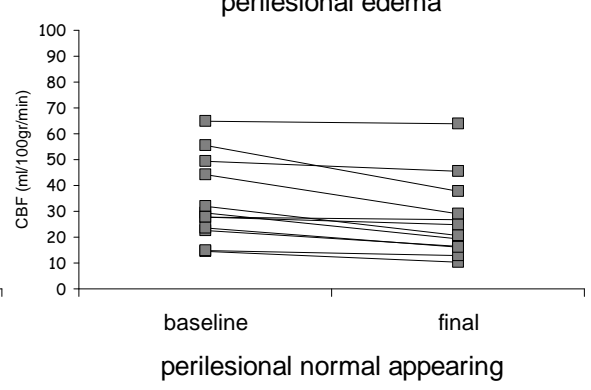

Fig. (47). Changes in rCBF in perilesional edematous area after cerebral perfusion pressure (CPP) elevation, induced by means of norephineprine infusion, during a double Xe-CT test. Patients were separated into those in whom a small improvement was obtained and into those in whom a paradoxically rCBF reduction was detected. These contusions have a low baseline value. In other contusions a reduction in rCBF was observed in association with higher baseline $\mathrm{rCBF}$ values. The changes in $\mathrm{rCBF}$ were less pronounced and less associated to baseline values in perilesional normal appearing area.

the $\mathrm{BBB}[126,127]$. In this situation, noradrenaline could result in a vasoconstrictory effect on sensitive and autoregulating arterioles [128]. This paradoxical reduction in $\mathrm{rCBF}$ was observed even in aneurysmal subarachnoid by Darby [129].

\section{Acute Subdural Hematoma}

Cerebral blood flow measurements in regions underlying acute SDH have rarely been obtained [102]. Before surgical evacuation, low CBF values can probably be found due to a local compressive effect with distortion and deeply reduced CPP [106]. After evacuation, complex mechanisms are involved and the cortex underlying the evacuated SDH is potentially at risk of further damage. Several CBF patterns can be found in the cortex beneath the evacuated SDH. In many cases, a traumatic contusion is associated and, in such cases, the consequences of SDH on $\mathrm{CBF}$ are mixed with those of traumatic contusion. However, in more isolated SDH, all 


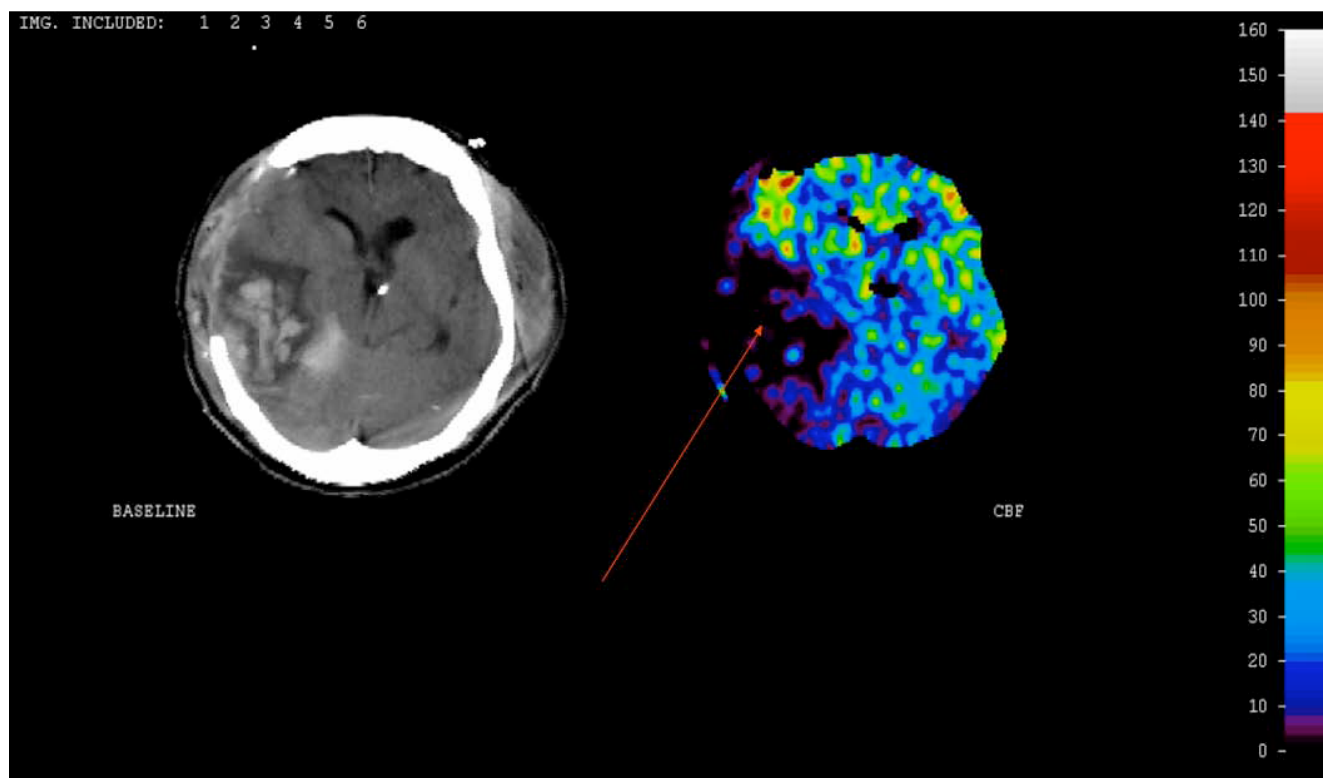

Fig. (48). Traumatic hematoma beneath an evacuated subdural hematoma (SDH). A wide area of hypoperfusion was associated with the traumatic hematoma.

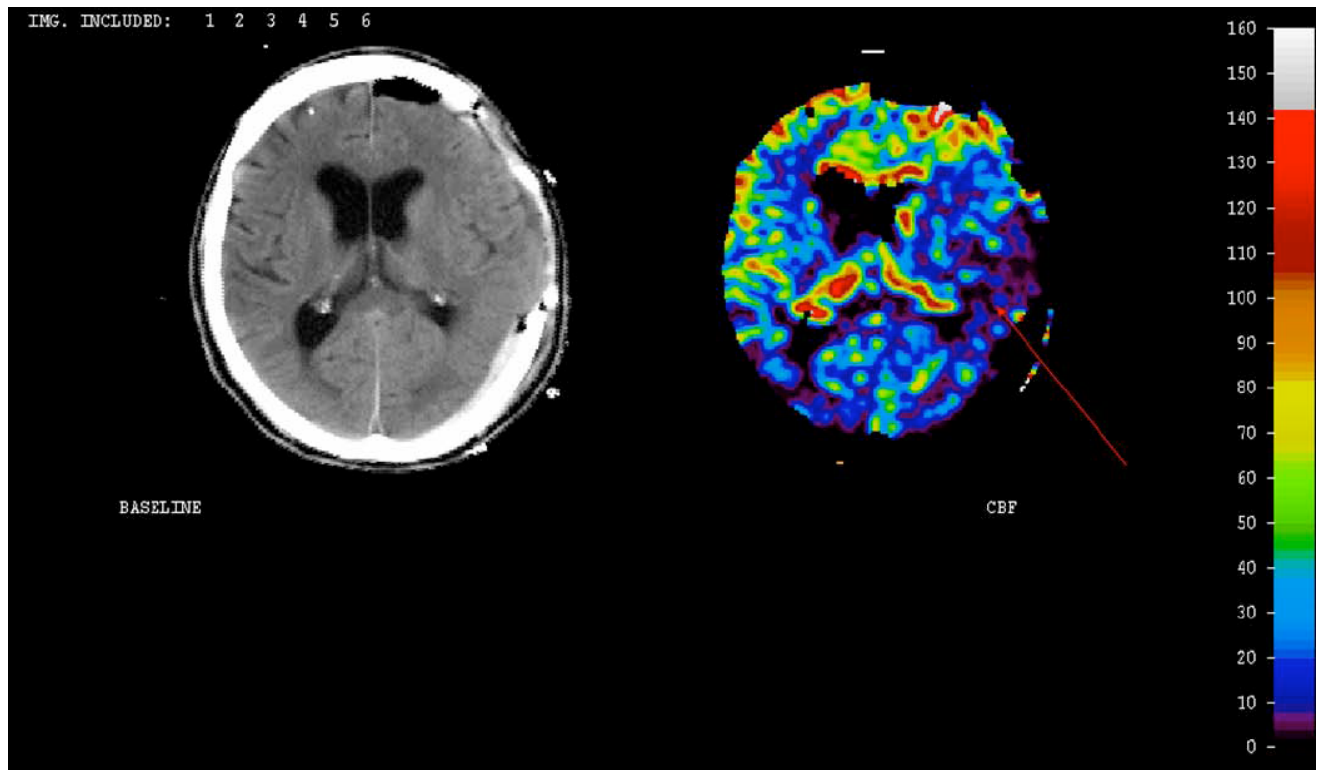

Fig. (49). Focal reduction of rCBF was found in normal appearing tissue beneath an evacuated subdural hematoma (SDH).

$\mathrm{CBF}$ ranges can be found beneath the evacuated SDH: a focal CBF depression, frequently due to associated intraparenchymal lesion (Fig. 48), but sometimes in apparently normal tissue (Fig. 49), or normal posttraumatic CBF reduction (Fig. 50), or a minor reduction in $\mathrm{rCBF}$, at least in comparison with contralateral side (Fig. 51), or a dense focal hyperemia with elevated values compared to contralateral hemisphere (Fig. 52). This finding had already been osserved by Marion [72] and Astrup [56], while our group found that hyperemia was frequent and prolonged only in patients with unfavourable outcome [44].

\section{The Future of Xe-CT in TBI Patients}

We believe that, in the next few years, TBI patients would be better characterized by means of integration between imaging and focal monitoring. While PET will probably remain dedicated to research, in clinical settings Mag- netic Resonance Imaging could provide a quantitative mapping of water distribution, the type of edema (cytotoxic or vasogenic) and the mapping of metabolites (Magnetic Resonance Spectroscopy), Xe-CT an accurate CBF mapping of $\mathrm{CBF}$, and $\mathrm{CT}$ perfusion information regarding $\mathrm{CBF}$ (semiquantitative), $\mathrm{CBV}$ and $\mathrm{BBB}$ permeability. These data, obtained as a snapshot, can be integrated with focal monitoring, $\mathrm{PtiO}_{2}$, TD-rCBF or microdialysis, especially if placed in the injured tissue [6-8]. Unfortunately, interpretation of regional monitoring is sometimes hypothetical because not coupled to rCBF measurement [130]. We can suppose that, in the future, as a consequence, an interplay between imaging and focal monitoring will become a daily occurrence: focal disturbance can be detected by imaging and guide the placement of focal monitoring. In return, data from focal monitoring should be validated by imaging that can test the value measured by focal monitoring by means of ROI ana- 


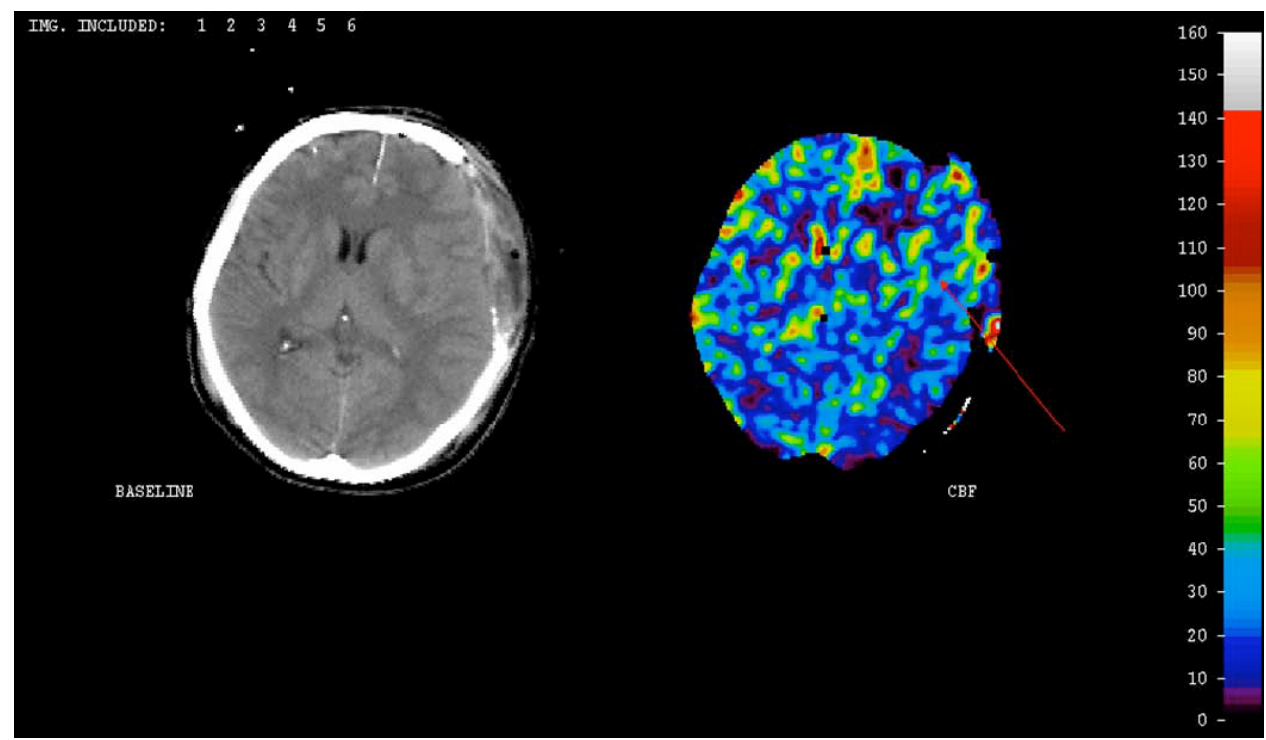

Fig. (50). Minor hypoperfusion, probably consistent with a normal posttraumatic CBF reduction, was found beneath an evacuated subdural hematoma $(\mathrm{SDH})$.

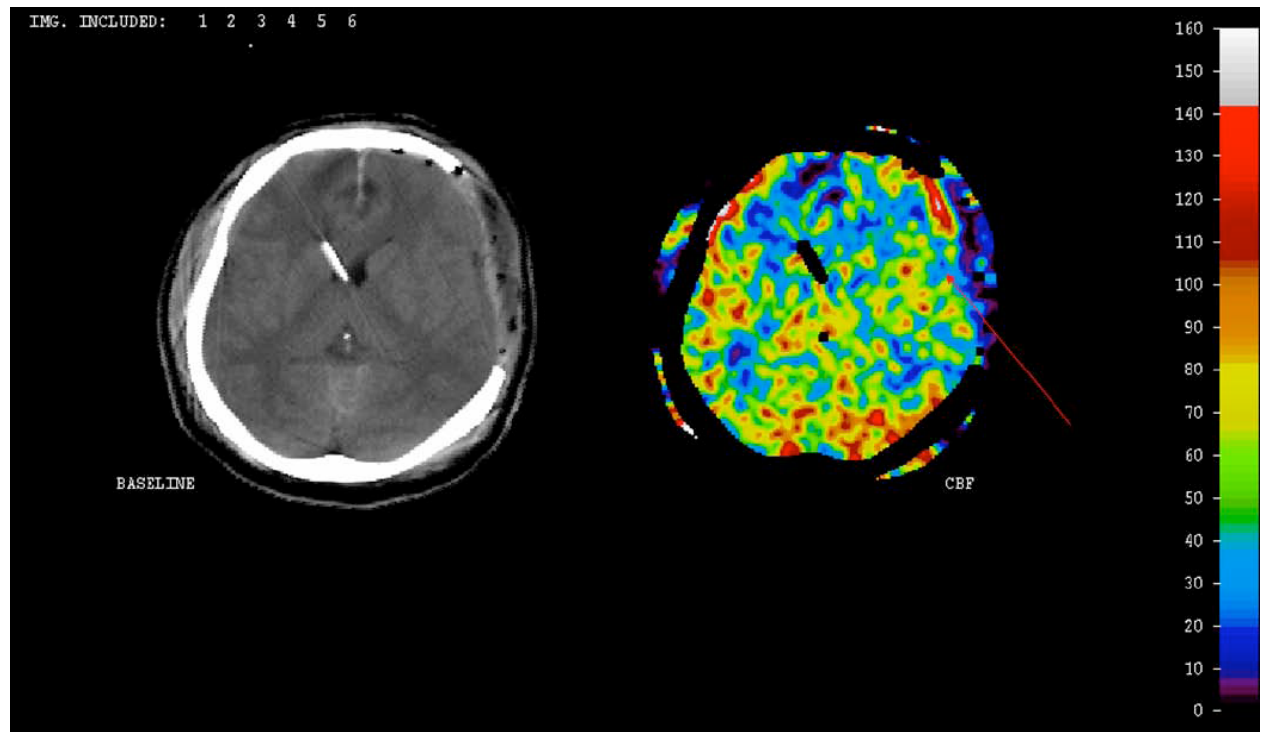

Fig. (51). Minimal hypoperfusion was found beneath an evacuated subdural hematoma (SDH), at least in comparison with contralateral normal appearing brain.

lyzing area surrounding the regional sensor tip (Fig. 53). Furthermore, imaging can evaluate whether the regional data is too regional. Once this cross validation is done, monitoring and management strategies could be applied at bedside and further imaging should be planned to verify the efficacy of therapies. These concepts have been debated by Valadka and Robertson in their recent review [131].

While only invasive regional monitoring can currently be used as a proxy or a direct measurement of regional $\mathrm{CBF}$, in the near future, even non invasive regional measurement of rCBF could be coupled and periodically validated by bedside measurement of quantitative rCBF by means of portable XeCT [28]. To date, however, most ICUs are located at a distance from neuroimaging: MRI is time consuming and CT perfusion calculations are still too complex and based on indirect assumptions. Fortunately, technology is moving CT to the patient, inside the ICU, instead of moving the patient to the CT [23]. Mobile CT has shown that it reduces time [132] and allows Xe-CT measurement at bedside [3]. The improved availability in neuro-ICU allows the clinician to measure $\mathrm{CBF}$ promptly whenever $\mathrm{CBF}$ reduction is suspected to be the cause of brain damage. As discussed above, the most important limitation of $\mathrm{Xe}-\mathrm{CT}$ measurement performed outside the ICU, is represented by the delay between the supposed CBF derangement and its detection, and the consequent delay in targeted therapies. Sturnegk et al. [133] showed that in such a way Xe-CT can be integrated into ICU management.

\section{CONCLUSION}

The Authors believe Xe-CT measurement in a neuroICU, located in a regional not university hospital, to be a useful tool which can improve our knowledge of CBF physi- 


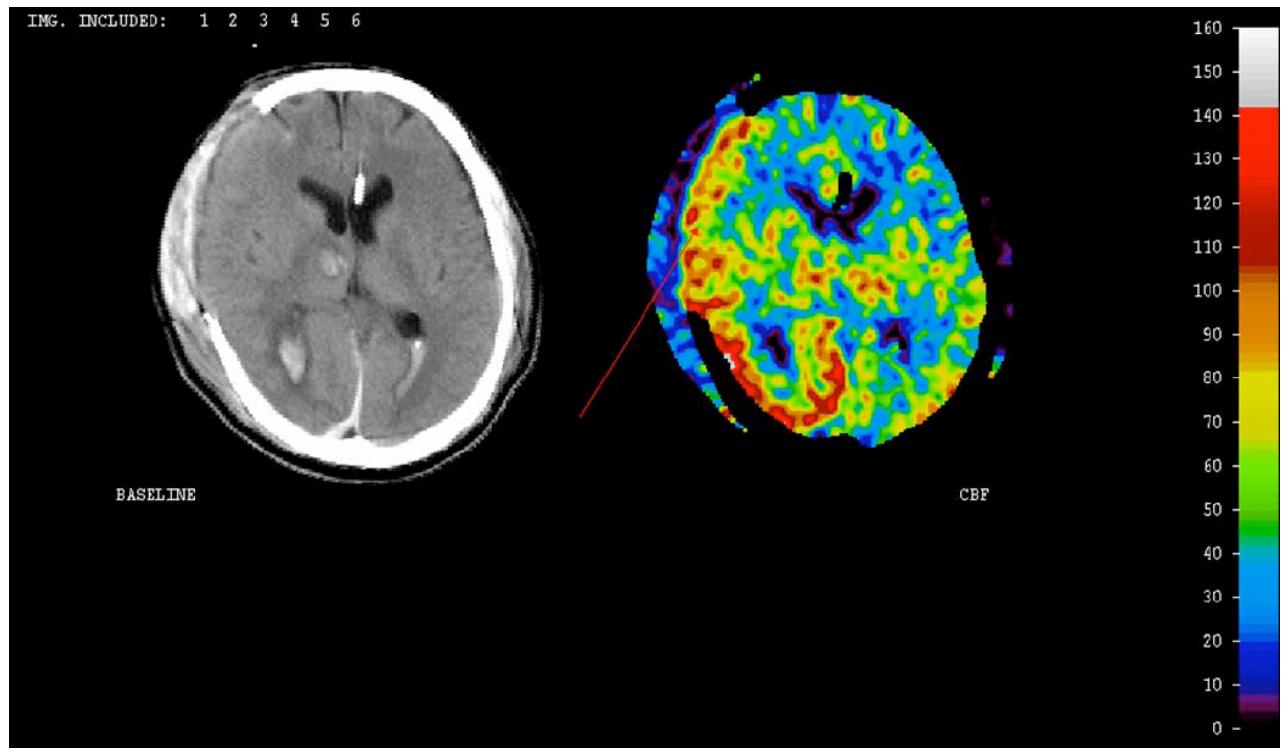

Fig. (52). Regional hyperemia was observed beneath an evacuated subdural hematoma (SDH).

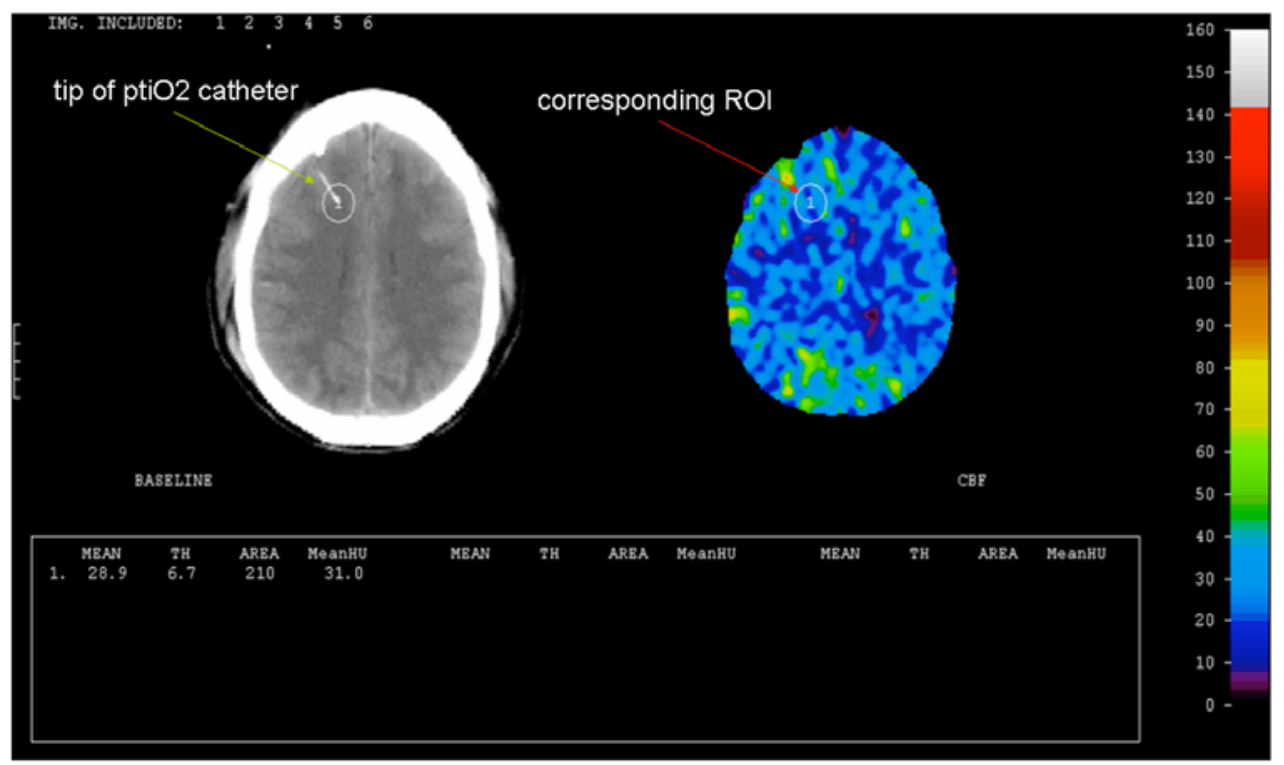

Fig. (53). Young patient with diffuse axonal injury. $\mathrm{A} \mathrm{Pt}_{\mathrm{i}} \mathrm{O} 2$ catheter was placed in apparently normal tissue in the frontal lobe. A circular region of interest (ROI) was placed to verify $\mathrm{rCBF}$ corresponding to the area surrounding the tip of the sensor.

ology and help to adapt management to fit the patient's needs.

The major limitation of Xe-CT imaging, that is the frequent delay in the prompt detection of rCBF decline before the development of an irreversible cause of ischemia, should be resolved in the near future with the diffusion of portable CT scanners. However, radioexposure remains a persistent limitation of Xe-CT.

\section{ACKNOWLEDGEMENTS}

We would like to thanks Howard Yonas, Department of Neurosurgery, University of New Mexico, Albuquerque, for his scientific support and Jerry Timpe and John Herron for their continuous technical guidance.

The authors thank Dr. Elizabeth Jenkins for helpful corrections of the manuscript.

\section{REFERENCES}

[1] Gur D, Good WF, Wolfson SK, Yonas H, Shabason L. In vivo mapping of local cerebral blood flow by xenon-enhanced computed tomography. Science 1981; 215: 1267-8.

[2] Yonas H, Gur D, Wolfson SK Jr, Good WF, Good BC, Latchaw RE. Xenon-enhanced computerised tomographic cerebral blood flow mapping. Lancet 1984; 1: 1357.

[3] Hillman J, Sturnegk P, Yonas H, et al. Bedside monitoring of CBF with xenon-CT and a mobile scanner: a novel method in neurointensive care. Br J Neurosurg 2005; 19: 395-401.

[4] Cunningham AS, Salvador R, Coles JP, et al. Physiological thresholds for irreversible issue damage in contusional regions following traumatic brain injury. Brain 2005; 128: 1931-42.

[5] Marshall LF. Head injury: recent past, present, and future. Neurosurgery 2000; 47: 546-61.

[6] Menzel M, Doppenberg EM, Zauner A, et al. Cerebral oxygenation in patients after severe head injury: monitoring and effects of arterial hyperoxia on cerebral blood flow, metabolism and intracranial pressure. J Neurosurg Anesthesiol 1999; 11: 240-51.

[7] Jaeger M, Soehle M, Schuhmann MU, Winkler D, Meixensberger J. Correlation of continuously monitored regional cerebral blood 
flow and brain tissue oxygen. Acta Neurochir (Wien) 2005; 147: 51-6.

[8] Vajkoczy P, Roth H, Horn P, et al. Continuous monitoring of regional cerebral blood flow: experimental and clinical validation of a novel thermal diffusion microprobe. J Neurosurg 2000; 93: 265 74.

[9] Coles JP. Imaging after brain injury. Br J Anaesth 2007; 99: 49-60.

[10] Kety SS, Schmidt CF. The nitrous oxide method for the quantitative determination of cerebral blood flow in man: theory, procedure and normal values. J Clin Invest 1948; 27: 476-83.

[11] Tone O, Ito U, Tomita H, Akimoto H, Sakemi H. Correlation between cerebral blood flow values obtained by Xenon/CT and KetySchmidt (N2O) methods. Acta Neurol Scand Suppl 1996; 166: 1821.

[12] Gur D, Yonas H, Jackson DL, et al. Simultaneous measurements of cerebral blood flow by the xenon/CT method and the microsphere method. A comparison. Invest Radiol 1985; 20: 672-7.

[13] Gur D, Yonas H, Jackson DL, et al. Measurement of cerebral blood flow during xenon inhalation as measured by the microspheres method. Stroke 1985; 16: 871-4.

[14] Pindzola RR, Yonas H. The xenon-enhanced computed tomography cerebral blood flow method. Neurosurgery 1998; 43: 1488-92.

[15] Kashiwagi S, Yamashita T: Inhalation protocols. In: Tomonaga M, Tanaka A, Yonas H, Eds. New York: Futura Publishing Company. Inc. 1995; pp. 67-71.

[16] Good WF, Gur D, Herron JM, Kennedy WH. The development of a xenon/computed tomography cerebral blood flow quality assurance phantom. Med Phys 1987; 14: 867-9.

[17] Segawa H. In: Tomonaga M, Tanaka A, Yonas H, Eds. Normative values. New York: Futura Publishing Company. Inc. 1995; pp. 6166.

[18] Zhang Z, Timpe G. Motion detection and correction xenonenhanced CT studies. Second International Conference on Xenon/CT CBF, Fukuoka, Japan 1992; pp. 23-6.

[19] Obrist WD, Thompson HK Jr, King CH, Wang HS. Determination of regional cerebral blood flow by inhalation of 133-xenon. Circ Res 1967; 20: 124-35.

[20] Obrist WD, Zihang Z, Yonas H. Effect of xenon-induced flow activation on xenon-enhanced computed tomography cerebral blood flow calculations. J Cereb Blood Flow Metab 1998; 18: 1192-5.

[21] Von Oettingen G, Bergholt B, Ostergaard L, Jensen LC, Gyldensted C, Astrup J. Xenon CT cerebral blood flow in patients with head injury: influence of pulmonary trauma on the input function. Neuroradiology 2000; 42: 168-73.

[22] Andrews PJD, Piper IR, Dearden NM, Miller JD. Secondary insults during intrahospital transport of head-injured patients. Lancet 1990; 335: 327-30.

[23] Pickard JD, Hutchinson PJ, Coles JP, et al. Imaging of cerebral blood flow and metabolism in brain injury in the ICU. Acta Neurochir Suppl 2005; 95: 459-64.

[24] Seifert H, Blass G, Leetz HK, Voges M. The radiation exposure of the patient from stable-xenon computed tomography. Br J Radiol 1995; 68: 301-5

[25] Becker H, Knoll M, Haubitz B, Rittmann KL. . In: Tomonaga M, Tanaka A, Yonas H, Eds. Radiation exposure. New York: Futura Publishing Company. Inc. 1995; pp. 55-60.

[26] Holl K, Nakano S, Yamashita T. In Tomonaga M, Tanaka A, Yonas H, Eds. The effects of stable Xenon gas. New York: Futura Publishing Company. Inc. 1995; pp. 41-53.

[27] Horn P, Vajkoczy P, Thomé C, Muench E, Schilling L, Schmiedek P. Xenon-induced flow activation in patients with cerebral insult who undergo xenon-enhanced CT blood flow studies. Am J Neuroradiol 2001; 22: 1543-9.

[28] Kim MN, Durduran T, Frangos S, et al. Noninvasive measurement of cerebral blood flow and blood oxygenation using near-infrared and diffuse correlation spectroscopies in critically brain-injured adults. Neurocrit Care 2010; 12: 173-80.

[29] Fatouros PP, Schroeder ML, Kuta AJ, et al. In: Tomonaga M, Tanaka A, Yonas H, Eds. Accuracy of low cerebral blood flow measurements using Xenon/computed tomography: effect of Xenon inhalation time. New York: Futura Publishing Company Inc. 1995; pp. 78-81.

[30] Marion DW, Crosby K. The effect of stable xenon on ICP. J Cereb Blood Flow Metab 1991; 11: 347-50.
[31] Ploughmann J, Astrup J, Pedersen J, Gyldensted C. Effect of stable xenon inhalation on intracranial pressure during measurement of cerebral blood flow in head injury. J Neurosurg 1994; 81: 822-8.

[32] Rosner MJ, Coley IB. Cerebral perfusion pressure, intracranial pressure, and head elevation. J Neurosurg 1986; 65: 636-41.

[33] Darby JM. In: Tomonaga M, Tanaka A, Yonas H, Eds. Applications of Xenon/computed tomography in intensive care. New York: Futura Publishing Company. Inc. 1995; pp. 333-47.

[34] Altman DG, Bland JM. Diagnostic tests. 1: sensitivity and specificity. BMJ 1994; 308: 1552 .

[35] Altman DG, Bland JM. Diagnostic tests. 2: predictive values. BMJ 1994; 309: 102.

[36] Von Oettingen G, Bergholt B, Gyldensted C, Astrup J. Blood flow and ischemia within traumatic cerebral contusions. Neurosurgery 2002; 50: 781-8; discussion 788-90.

[37] Schroder ML, Muizelaar JP, Kuta AJ, Choi SC. Thresholds for cerebral ischemia after severe head injury: relationship with late CT findings and outcome. J Neurotrauma 1996; 13: 17-23.

[38] Muizelaar JP, Schröder ML. Overview of monitoring of cerebral blood flow and metabolism after severe head injury. Can J Neurol Sci 1994; 21: S6-11.

[39] Chieregato A, Tanfani A, Noto A, Fronza S, Cocciolo F, Fainardi E. Cerebral blood flow thresholds predicting new hypoattenuation areas due to macrovascular ischemia during the acute phase of severe and complicated aneurysmal subarachnoid hemorrhage. A preliminary study. Acta Neurochir Suppl 2008; 102: 311-6.

[40] Robertson CS, Cormio M. Cerebral metabolic management. New Horiz 1995; 3: 410-22.

[41] Cormio M, Valadka AB, Robertson CS. Elevated jugular venous oxygen saturation after severe head injury. J Neurosurg 1999; 90 : 9-15

[42] Cormio M, Gopinath SP, Valadka A, Robertson CS. Cerebral hemodynamic effects of pentobarbital coma in head-injured patients. J Neurotrauma 1999; 16: 927-36.

[43] Botteri M, Bandera E, Minelli C, Latronico N. Cerebral blood flow thresholds for cerebral ischemia in traumatic brain injury. A systematic review. Crit Care Med 2008; 36: 3089-92.

[44] Chieregato A, Noto A, Tanfani A, Bini G, Martino C, Fainardi E. Hyperemia beneath evacuated acute subdural hematoma is frequent and prolonged in patients with an unfavorable outcome: a xecomputed tomographic study. Neurosurgery 2009; 64: 705-17; discussion 717-8.

[45] Kelly DF, Kordestani RK, Martin NA, et al. Hyperemia following traumatic brain injury: relationship to intracranial hypertension and outcome. J Neurosurg 1996; 85: 762-71.

[46] Kelly DF, Martin NA, Kordestani R, et al. Cerebral blood flow as a predictor of outcome following traumatic brain injury. J Neurosurg 1997; 86: 633-41.

[47] Yonas H, Gur D, Claassen D, Wolfson SK Jr, Moossy J. Stable xenon enhanced computed tomography in the study of clinical and pathologic correlates of focal ischemia in baboons. Stroke 1988; 19: $228-38$

[48] Yonas H, Gur D, Claassen D, Wolfson SK Jr, Moossy J. Stable xenon-enhanced CT measurement of cerebral blood flow in reversible focal ischemia in baboons. J Neurosurg 1990; 73: 266-73.

[49] Jones TH, Morawetz RB, Crowell RM, et al. Thresholds of focal cerebral ischemia in awake monkeys. J Neurosurg 1981; 54: 773782 .

[50] Kaufmann AM, Firlik AD, Fukui MB, Wechsler LR, Jungries CA Yonas H. Ischemic core and penumbra in human stroke. Stroke 1999; 30: 93-9.

[51] Obrist WD, Langfitt TW, Jaggi JL, Cruz J, Gennarelli TA. Cerebral blood flow and metabolism in comatose patients with acute head injury. Relationship to intracranial hypertension. J Neurosurg 1984; 61: 241-53.

[52] Cruz J, Miner ME. In: Miner ME,Wagner KA, Eds. Modulating cerebral oxygen delivery and extraction in acute traumatic coma. Boston: Butterworths, 1986; pp. 55-72.

[53] McLaughlin MR, Marion DW. Cerebral blood flow and vasoresponsivity within and around cerebral contusions. J Neurosurg 1996; 85: 871-6.

[54] Marion DW, Bouma GJ. The use of stable xenon-enhanced computed tomographic studies of cerebral blood flow to define changes in cerebral carbon dioxide vasoresponsivity caused by a severe head injury. Neurosurgery 1991; 29: 869-73. 
[55] Skippen P, Seear M, Poskitt K, et al. Effect of hyperventilation on regional cerebral blood flow in head-injured children. Crit Care Med 1997; 25: 1402-9.

[56] Astrup J, Bergholt B. In: Tomonaga M, Tanaka A, Yonas H, Eds. Management of patients with severe head injury. New York: Futura Publishing Company. Inc. 1995: pp. 267-274.

[57] Chieregato A, Tanfani A, Compagnone C, Pascarella R, Targa L, Fainardi E. Cerebral blood flow in traumatic contusions is predominantly reduced after an induced acute elevation of cerebral perfusion pressure. Neurosurgery 2007; 60: 115-2; discussion 123.

[58] Hlatky R, Contant CF, Diaz-Marchan P, Valadka AB, Robertson CS. Significance of a reduced cerebral blood flow during the first 12 hours after traumatic brain injury. Neurocrit Care 2004; 1: 6983.

[59] Bouma GJ, Muizelaar JP, Stringer WA, Choi SC, Fatouros P, Young HF. Ultra-early evaluation of regional blood flow in severely head-injured patients using xenon-enhanced computerized tomography. J Neurosurg 1992; 77: 360-8.

[60] Matta BF, Lam AM. The rate of blood withdrawal affects the accuracy of jugular venous bulb. Oxygen saturation measurements. Anesthesiology 1997; 86: 806-8.

[61] Gur D, Yonas H, Good WF. Local cerebral blood flow by xenonenhanced CT: current status, potential improvements, and future directions. Cerebrovasc Brain Metab Rev 1989; 1: 68-86.

[62] Damasio H. A computed tomographic guide to the identification of cerebral vascular territories. Arch Neurol 1983; 40: 138-42.

[63] Yonas H, Darby JM, Marks EC, Durham SR, Maxwell C. CBF measured by Xe-CT: approach to analysis and normal values. J Cereb Blood Flow Metab 1991; 11: 716-25

[64] Bell BA, Symon L, Branston NM. CBF and time thresholds for the formation of ischemic cerebral edema, and effect of reperfusion in baboons. J Neurosurg 1985; 62: 31-41.

[65] Wintermark M, Lepori D, Cotting J, et al. Brain perfusion in children: evolution with age assessed by quantitative perfusion computed tomography. Pediatrics 2004; 113: 1642-52.

[66] Suzuki K. The changes of regional blood flow with advancing age in normal children. Nagoya Med J 1990; 34: 159-70.

[67] Zwienenberg M, Muizelaar JP. Severe pediatric head injury: the role of hyperemia revisited. J Neurotrauma 1999; 16: 937-43.

[68] Yamaguchi T, Kanno I, Uemura K, et al. Reduction in regional cerebral metabolic rate of oxygen during human aging. Stroke 1986; 17: 1220-8.

[69] Jensen K, Freundlich M, Bunemann L, Therkelsen K, Hansen H, Cold GE. The effect of indomethacin upon cerebral blood flow in healthy volunteers. Acta Neurochir 1993; 124: 114-9.

[70] Datsur DK, Lane MH, Hansen DB, et al. In: Birren JE, Butler RN, Greenhouse SW, Eds. Effects of aging on cerebral circulation and metabolism in man. in human aging. A biological and behavioral study: Washington DC, U.S. Government Printing Office 1963; pp. 59-76.

[71] Lerou JG. Nomogram to estimate age-related MAC. Br J Anaesth 2004; 93: 288-91.

[72] Marion DW, Darby J, Yonas H. Acute regional cerebral blood flow changes caused by severe head injury. J Neurosurg 1991; 74: 40714.

[73] Marion DW. In: Tomonaga M, Tanaka A, Yonas H, Eds. Changes in the management of severe traumatic brain injury resulting from early cerebral blood flow information. New York: Futura Publishing Company. Inc. 1995; pp. 283-7.

[74] Martin NA, Patwardhan RV, Alexander MJ, et al. Characterization of cerebral hemodynamic phases following severe head trauma: hypoperfusion, hyperemia, and vasospasm. J Neurosurg 1997; 87: 9-19.

[75] Chieregato A, Fainardi E, Servadei F, et al. Centrifugal distribution of regional cerebral blood flow and its time course in traumatic intracerebral hematomas. J Neurotrauma 2004; 21: 655-66.

[76] Soustiel JF, Glenn TC, Shik V, Boscardin J, Mahamid E, Zaaroor M. Monitoring of cerebral blood flow and metabolism in traumatic brain injury. J Neurotrauma 2005; 22: 955-65.

[77] Adelson PD, Clyde B, Kochanek PM, Wisniewski SR, Marion DW, Yonas H. Cerebrovascular response in infants and young children following severe traumatic brain injury: a preliminary report. Pediatr Neurosurg 1997; 26: 200-7.

[78] Sharples PM, Stuart AG, Matthews DSF, Aynsley-Green A, Eyre JA. Cerebral blood flow and metabolism in children with severe head injury. Part 1: relation to age, Glasgow coma score, outcome, intracranial pressure, and time after injury. J Neurol Neurosurg Psychiatry $1995 ; 58: 145-52$.

[79] Alexander MJ, Martin NA, Khanna R, Caron M, Becker DP. Regional cerebral blood flow trends in head injured patients with focal contusions and cerebral edema. Acta Neurochir Suppl 1994; 60: 479-81.

[80] Bouma GJ, Muizelaar JP, Choi SC, Newlon PG, Young HF. Cerebral circulation and metabolism after severe traumatic brain injury: the elusive role of ischemia. J Neurosurg 1991; 75: 685-93.

[81] Bouma GJ, Muizelaar JP. Cerebral blood flow in severe clinical head injury. New Horiz 1995; 3: 384-94.

[82] Glenn TC, Kelly DF, Boscardin WJ, et al. Energy dysfunction as a predictor of outcome after moderate or severe head injury: indices of oxygen, glucose, and lactate metabolism. J Cereb Blood Flow Metab 2003; 23: 1239-50.

[83] Vespa P, Bergsneider M, Hattori N, et al. Metabolic crisis without brain ischemia is common after traumatic brain injury: a combined microdialysis and positron emission tomography study. J Cereb Blood Flow Metab 2005; 25: 763-74.

[84] Coles JP, Fryer TD, Smielewski P, et al. Defining ischemic burden after traumatic brain injury using 150 PET imaging of cerebral physiology. J Cereb Blood Flow Metab 2004; 24: 191-201.

[85] Coles JP, Fryer TD, Smielewski P, et al. Incidence and mechanisms of cerebral ischemia in early clinical head injury. $\mathrm{J}$ Cereb Blood Flow Metab 2004; 24: 202-11.

[86] Bergsneider M, Hovda DA, Shalmon E, et al. Cerebral hyperglycolysis following severe traumatic brain injury in humans: a positron emission tomography study. J Neurosurg 1997; 86: 241-51.

[87] Alkire MT. Quantitative EEG correlations with brain glucose metabolic rate during anesthesia in volunteers. Anesthesiology 1998; 89: 323-33.

[88] Pinaud M, Lelasque JN, Chetanneau A, Fauchoux N, Menegalli D, Souron R. Effects of propofol on cerebral hemodynamics and metabolism in patients with brain trauma. Anesthesiology 1990; 73: 404-9.

[89] Johnston AJ, Steiner LA, Chatfield DA, et al. Effects of propofol on cerebral oxygenation and metabolism after head injury. $\mathrm{Br} \mathrm{J}$ Anaesth 2003; 91: 781-6.

[90] Stocchetti N, Rossi S, Buzzi F, Mattioli C, Paparella A, Colombo A. Intracranial hypertension in head injury: management and results. Intensive Care Med 1999; 25: 371-6.

[91] Olivecrona M, Zetterlund B, Rodling-Wahlström M, Naredi S, Koskinen LO. Absence of electroencephalographic seizure activity in patients treated $\mathrm{f}$ head injury with an intracranial pressuretargeted therapy. J Neurosurg 2009; 110: 300-5.

[92] Stocchetti N, Pagan F, Calappi E, et al. Inaccurate early assessment of neurological severity in head injury. J Neurotrauma 2004; 21 : 1131-40.

[93] Balestreri M, Czosnyka M, Chatfield DA, et al. Predictive value of Glasgow Coma Scale after brain trauma: change in trend over the past ten years. J Neurol Neurosurg Psychiatry 2004; 75: 161-2.

[94] Masaoka H, Takasato Y, Hayakawa T, et al. Cerebral blood flow measurement of severely head-injured patients during mild hypothermia. Keio J Med 2000; 49(Suppl 1): A159-60.

[95] Chieregato A, Tanfani A, Compagnone C, et al. Global cerebral blood flow and CPP after severe head injury: a xenon-CT study. Intensive Care Med 2007; 33: 856-62.

[96] Cruz J, Jaggi JL, Hoffstad OJ. Cerebral blood flow, vascular resistance, and oxygen metabolism in acute brain trauma: redefining the role of cerebral perfusion pressure. Crit Care Med 1995; 23: 1412-7.

[97] Shalmon E, Caron MJ, Martin NA, Blander ES, Hovda DA, Becker DP. In: Nagai H, Kamiya K, Ishii S, Eds. The relationship between cerebral blood flow and metabolism in severely head injured patients. Tokyo: Springer, 1994; pp. 348-52.

[98] Robertson CS, Narayan RK, Gokaslan ZL, et al. Cerebral arterovenous differences as an estimate of cerebral blood flow in comatose patients. J Neurosurg 1989; 70: 222-30.

[99] Stocchetti N, Zanaboni C, Colombo A, et al. Refractory intracranial hypertension and "second-tier" therapies in traumatic brain injury. Intensive Care Med 2008; 34: 461-7.

[100] Jennett B, Bond M. Assessment of outcome after severe brain damage. A practical scale. Lancet 1975; 1: 480-4

[101] Stocchetti N, Canavesi K, Magnoni S, et al. Arterio-jugular difference of oxygen content and outcome after head injury. Anesth Analg 2004; 99: 230-4. 
[102] Schroder ML, Muizelaar JP, Kuta AJ. Documented reversal of global ischemia immediately after removal of an acute subdural hematoma. J Neurosurg 1994; 80: 324-7.

[103] Marino R, Gasparotti R, Pinelli L, et al. Posttraumatic cerebral infarction in patients with moderate or severe head trauma. Neurology 2006; 67: 1165-71.

[104] Furuya Y, Hlatky R, Valadka AB, Diaz P, Robertson CS. Comparison of cerebral blood flow in computed tomographic hypodense areas of the brain in head-injured patients. Neurosurgery 2003; 52 : 340-6.

[105] Marshall LF, Gautille T. Large and small "holes" in the brain: reversible or irreversible changes in head injury. Acta Neurochir Suppl (Wien) 1990; 51: 300-1.

[106] Verweij BH, Muizelaar JP, Vinas FC. Hyperacute measurement of intracranial pressure, cerebral perfusion pressure, jugular venous oxygen saturation, and laser Doppler flowmetry, before and during removal of traumatic acute subdural hematoma. J Neurosurg 2001; 95: 569-72.

[107] Soustiel JF, Mahamid E, Goldsher D, Zaaroor M. Perfusion-CT for early assessment of traumatic cerebral contusions. Neuroradiology 2008; 50: 189-96.

[108] Schroder ML, Muizelaar JP, Bullock MR, Salvant JB, Povlishock JT. Focal ischemia due to traumatic contusions documented by stable xenon-CT and ultrastructural studies. J Neurosurg 1995; 82: 966-71.

[109] Hoelper BM, Reinert MM, Zauner A, Doppenberg E, Bullock R. rCBF in hemorrhagic, non-hemorrhagic and mixed contusions after severe head injury and its effect on perilesional cerebral blood flow. Acta Neurochir Suppl 2000; 76: 21-5.

[110] Marmarou A, Signoretti S, Fatouros PP, Portella G, Aygok GA, Bullock MR. Predominance of cellular edema in traumatic brain swelling in patients with severe head injuries. J Neurosurg 2006; 104: 720-30

[111] Ribas GC, Jane JA. Traumatic contusions and intracerebral hematomas. J Neurotrauma 1992; 9: S265-8.

[112] Chieregato A, Compagnone C, Tanfani A, et al. Cerebral blood flow mapping in two different subtypes of intraparenchymal hemorrhagic traumatic lesions. Acta Neurochir Suppl 2005; 95: 159-64.

[113] Chieregato A, Fainardi E. Potential role of surgery in traumatic focal brain lesions as revealed by functional imaging. Intensive Care Med 2008; 34: 2125-6.

[114] Kawamata T, Katayama Y, Mori T, Aoyama N, Tsubokawa T. Mechanisms of the mass effect of cerebral contusion: ICP monitoring and diffusion MRI study. Acta Neurochir Suppl 2002; $81 ; 281-83$.

[115] Golding EM, Robertson CS, Fitch JC, Goodman JC, Bryan RM Jr. Segmental vascular resistance after mild controlled cortical impact injury in the rat. J Cereb Blood Flow Metab 2003; 23: 210-8.

[116] Rodríguez-Baeza A, Reina-de la Torre F, Poca A, Martí M, Garnacho A. Morphological features in human cortical brain microvessels after head injury: a three-dimensional and immunocytochemical study. Anat Rec A Discov Mol Cell Evol Biol 2003; 273: 58393

[117] Menon DK, Coles JP, Gupta AK, et al. Diffusion limited oxygen delivery following head injury. Crit Care Med 2004; 32: 1384-90.
[118] Yoshihara M, Bandoh K, Marmarou A. Cerebrovascular carbon dioxide reactivity assessed by intracranial pressure dynamics in severely head injured patients. J Neurosurg 1995; 82: 386-93.

[119] Diringer MN, Videen TO, Yundt K, et al. Regional cerebrovascular and metabolic effects of hyperventilation after severe traumatic brain injury. J Neurosurg 2002; 96: 103-8.

[120] Rosner MJ, Daughton S. Cerebral perfusion pressure management in head injury. J Trauma 1990; 30: 933-41.

[121] Rosner MJ, Rosner SD, Johnson AH. Cerebral perfusion pressure: management protocol and clinical results. J Neurosurg 1995; 83: 949-62.

[122] Zhuang J, Schmoker D, Shackford SR, Pietropaoli JA. Focal brain injury results in severe cerebral ischemia despite maintenance of cerebral perfusion pressure. J Trauma 1992; 33: 83-8.

[123] Steiner LA, Coles JP, Johnston AJ, et al. Responses of posttraumatic pericontusional cerebral blood flow and blood volume to an increase in cerebral perfusion pressure. J Cereb Blood Flow Metab 2003; 23: 1371-7.

[124] Rapoport SI. In: Welch KMA, Reis DJ, Caplan LR, Siesjo BK, Weir B, Eds. Brain Edema and the Blood-Brain Barrier. San Diego: Academic Press 1997; pp. 25-8.

[125] Johansson BB. In Welch KMA, Reis DJ, Caplan LR, Siesjo BK, Weir B, Eds. Hypertension. San Diego: Academic Press 1997, pp. $142-44$.

[126] Chiueh CC, Sun CL, Kopin IJ, Fredericks WR, Rapoport SI. Entry of [3H]norepinephrine, [125I] albumin and Evans blue from blood into brain following unilateral osmotic opening of the blood-brain barrier. Brain Res 1978; 145: 291-301.

[127] Hardebo JE, Edvinsson L, Mackenzie ET, Owman C. Regional brain uptake of noradrenaline following mechanical or osmotic opening of the blood-brain barrier. Acta Physiol Scand 1977; 101: 342-350.

[128] Toda N. Influence of dopamine and noradrenaline on isolated arteries of the dog. Br J Pharmacol 58: 121-126, 1976.

[129] Darby JM, Yonas H, Marks EC, Durham S, Snyder RW, Nemoto EM. Acute cerebral blood flow response to dopamine-induced hypertension after subarachnoid hemorrhage. J Neurosurg 1994; 80: 857-64.

[130] Longhi L, Pagan F, Valeriani V, et al. Monitoring brain tissue oxygen tension in brain-injured patients reveals hypoxic episodes in normal-appearing and in peri-focal tissue. Intensive Care Med 2007; 33: 2136-42.

[131] Valadka AB, Robertson CS. Surgery of cerebral trauma and associated critical care. Neurosurgery 2007; 61(1 Suppl): 203-20; discussion 220-1.

[132] Gunnarsson T, Theodorsson A, Karlsson P, et al. Mobile computerized tomography scanning in the neurosurgery intensive care unit increase in patient safety and reduction of staff workload. J Neurosurg 2000; 93: 432-6.

[133] Sturnegk P, Mellergård P, Yonas H, Theodorsson A, Hillman J. Potential use of quantitative bedside CBF monitoring (Xe-CT) for decision making in neurosurgical intensive care. $\mathrm{Br} \mathrm{J}$ Neurosurg 2007; $21: 332-9$.

(c) Chieregato et al.; Licensee Bentham Open.

This is an open access article licensed under the terms of the Creative Commons Attribution Non-Commercial License (http://creativecommons.org/licenses/by-nc/3.0/) which permits unrestricted, non-commercial use, distribution and reproduction in any medium, provided the work is properly cited. 